\title{
Integration of collinear-type doubly unresolved counterterms in NNLO jet cross sections
}

\author{
Vittorio Del Duca, ${ }^{a}$ Gábor Somogyi ${ }^{b}$ and Zoltán Trócsányi ${ }^{c}$ \\ ${ }^{a}$ Istituto Nazionale di Fisica Nucleare, Laboratori Nazionali di Frascati, \\ Via E. Fermi 40, I-00044 Frascati, Italy \\ ${ }^{b}$ PH Department, TH Unit, CERN, \\ CH-1211 Geneva 23, Switzerland \\ ${ }^{c}$ University of Debrecen and MTA-DE Particle Physics Research Group, \\ H-4010 Debrecen, PO Box 105, Hungary \\ E-mail: Vittorio.DelDuca@lnf.infn.it, Gabor.Somogyi@cern.ch, \\ Zoltan. Trocsanyi@cern.ch
}

ABSTRACT: In the context of a subtraction method for jet cross sections at NNLO accuracy in the strong coupling, we perform the integration over the two-particle factorised phase space of the collinear-type contributions to the doubly unresolved counterterms. We present the final result as a convolution in colour space of the Born cross section and of an insertion operator, which is written in terms of master integrals that we expand in the dimensional regularisation parameter.

KeYwords: QCD Phenomenology, Jets

ARXIV EPRINT: 1301.3504 


\section{Contents}

1 Introduction 2

2 Notation 3

2.1 Matrix elements 4

2.2 Cross sections 4

2.3 Momentum mappings and phase-space factorisation 5

2.4 Kinematic variables 6

$\begin{array}{lll}3 & \text { Integrating the doubly unresolved approximate cross section } & 7\end{array}$

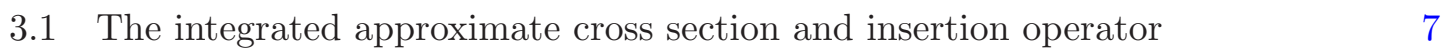

$\begin{array}{lll}3.2 & \text { Flavour-summed integrated counterterms } & 9\end{array}$

4 Integrated counterterms $\quad 11$

4.1 Integrated triple collinear counterterm 11

$\begin{array}{lll}4.2 & \text { Integrated double collinear counterterm } & 12\end{array}$

$\begin{array}{lll}4.3 & \text { Integrated soft collinear counterterms } & 13\end{array}$

5 Results $\quad \mathbf{1 5}$

$\begin{array}{lll}5.1 & \text { Integrated counterterms to } \mathrm{O}\left(\epsilon^{-2}\right) & 15\end{array}$

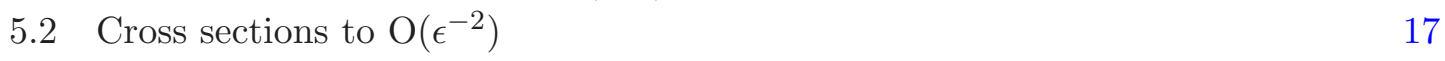

6 Conclusions 24

$\begin{array}{ll}\text { A Modified doubly real subtraction terms } & 24\end{array}$

$\begin{array}{ll}\text { B Spin-averaged splitting kernels } & \mathbf{2 5}\end{array}$

B.1 Two-parton kernels 26

$\begin{array}{ll}\text { B.2 Three-parton kernels } & 26\end{array}$

C Integrating the triple collinear counterterm $\quad 30$

$\begin{array}{ll}\text { C.1 Master integrals } & 30\end{array}$

$\begin{array}{lll}\text { C.2 Explicit representations } & 31\end{array}$

$\begin{array}{ll}\text { D Integrating the double collinear counterterm } & 35\end{array}$

D.1 Master integrals $\quad 35$

D.2 Explicit representation 36

$\begin{array}{lll}\text { E Integrating the soft collinear counterterms } & 37\end{array}$

$\begin{array}{lll}\text { E.1 Master integrals } & 37\end{array}$

$\begin{array}{lll}\text { E.2 } & \text { Explicit representations } & 39\end{array}$ 


\section{Introduction}

The high precision of the experimental measurements at the LHC calls for an evaluation of the jet, heavy-quark, vector-boson and Higgs-boson production rates at hadron colliders which is at least as precise. In recent years, we have witnessed fast progress in the evaluation of the production rates mentioned above, in association with many jets, at next-to-leading order (NLO) accuracy in the strong coupling constant $\alpha_{\mathrm{s}}$. That rested upon efficient methods to compute one-loop amplitudes with many legs, and upon subtraction algorithms to evaluate QCD cross sections at NLO at the partonic level [1-5], or at the hadronic level, through interfaces, like MC@NLO [6] or POWHEG [7-9] with parton-shower event generators. Such algorithms are based on the universality - i.e., on the independence from a specific scattering process - of the infrared emissions.

Lately, the evaluation of production rates at next-to-next-to-leading order (NNLO) accuracy has received a lot of attention. Fully differential cross sections for vectorboson [10, 11], Higgs-boson [12, 13], diphoton [14] and Higgs-vector-boson [15] production have been evaluated, and the computation of the NNLO corrections to top-pair production is well under way [16-18]. Much work has gone into the lay-out of a general subtraction algorithm to compute cross sections at NNLO accuracy [5, 19-37]. The antenna scheme [21] has yielded the evaluation of total rates [38-41] and event shapes [42-46] in electron-positron annihilation, leading to precise determinations of the strong coupling [47-50].

No completely general subtraction algorithm to compute cross sections at NNLO accuracy, though, has been devised yet. In order to do that, one must define subtraction terms that properly regularise the real-emission phase-space integrals and then one must combine the integrated form of those counterterms with the virtual contributions, so as to cancel the infrared divergences of the loop amplitudes, in such a way that the cancellation of both the kinematic singularities in the real-emission pieces and the explicit $\epsilon$-poles in the virtual pieces be local. This implies that the subtraction terms and the real-emission contributions must tend to the same value in all kinematic limits where the latter diverge, and that the cancellation of explicit $\epsilon$-poles between the integrated subtraction terms and the virtual contributions must take place point-wise in phase space. In particular, that implies that it is possible to write the integrated counterterms in such a way that they can be explicitly combined with virtual contributions, before phase-space integration. Practically, the locality of the subtraction scheme is also important to ensure good numerical efficiency of the algorithm. In broad outline, we remind how this occurs at NLO. After fixing the leading-order $m$-jet cross section as the integral of the fully differential Born cross section $\mathrm{d} \sigma_{m}^{\mathrm{B}}$ of $m$ final-state patrons over the available $m$-parton phase space defined by the jet function $J_{m}$,

$$
\sigma^{\mathrm{LO}}=\int_{m} \mathrm{~d} \sigma_{m}^{\mathrm{B}} J_{m}
$$

the NLO correction,

$$
\sigma^{\mathrm{NLO}}=\int_{m+1} \mathrm{~d} \sigma_{m+1}^{\mathrm{R}} J_{m+1}+\int_{m} \mathrm{~d} \sigma_{m}^{\mathrm{V}} J_{m}
$$


can be rewritten as a sum of finite integrals,

$$
\sigma^{\mathrm{NLO}}=\int_{m+1} \mathrm{~d} \sigma_{m+1}^{\mathrm{NLO}}+\int_{m} \mathrm{~d} \sigma_{m}^{\mathrm{NLO}}
$$

where each of the two terms on the right-hand side,

$$
\begin{aligned}
& \mathrm{d} \sigma_{m+1}^{\mathrm{NLO}}=\left[\mathrm{d} \sigma_{m+1}^{\mathrm{R}} J_{m+1}-\mathrm{d} \sigma_{m+1}^{\mathrm{R}, \mathrm{A}_{1}} J_{m}\right]_{\epsilon=0}, \\
& \mathrm{~d} \sigma_{m}^{\mathrm{NLO}}=\left[\left(\mathrm{d} \sigma_{m}^{\mathrm{V}}+\int_{1} \mathrm{~d} \sigma_{m+1}^{\mathrm{R}, \mathrm{A}_{1}}\right) J_{m}\right]_{\epsilon=0},
\end{aligned}
$$

is integrable in four dimensions by construction [1-5].

Likewise, we can write the NNLO correction to the cross section as a sum of three contributions, the doubly unresolved, the one-loop singly unresolved, and the two-loop doubly virtual terms,

$$
\sigma^{\mathrm{NNLO}}=\int_{m+2} \mathrm{~d} \sigma_{m+2}^{\mathrm{RR}} J_{m+2}+\int_{m+1} \mathrm{~d} \sigma_{m+1}^{\mathrm{RV}} J_{m+1}+\int_{m} \mathrm{~d} \sigma_{m}^{\mathrm{VV}} J_{m}
$$

and rearrange it as follows,

$$
\sigma^{\mathrm{NNLO}}=\int_{m+2} \mathrm{~d} \sigma_{m+2}^{\mathrm{NNLO}}+\int_{m+1} \mathrm{~d} \sigma_{m+1}^{\mathrm{NNLO}}+\int_{m} \mathrm{~d} \sigma_{m}^{\mathrm{NNLO}}
$$

where each of the three terms on the right-hand side,

$$
\begin{aligned}
& \mathrm{d} \sigma_{m+2}^{\mathrm{NNLO}}=\left\{\mathrm{d} \sigma_{m+2}^{\mathrm{RR}} J_{m+2}-\mathrm{d} \sigma_{m+2}^{\mathrm{RR}, \mathrm{A}_{2}} J_{m}-\left[\mathrm{d} \sigma_{m+2}^{\mathrm{RR}, \mathrm{A}_{1}} J_{m+1}-\mathrm{d} \sigma_{m+2}^{\mathrm{RR}, \mathrm{A}_{12}} J_{m}\right]\right\}_{\epsilon=0}, \\
& \mathrm{~d} \sigma_{m+1}^{\mathrm{NNLO}}=\left\{\left[\mathrm{d} \sigma_{m+1}^{\mathrm{RV}}+\int_{1} \mathrm{~d} \sigma_{m+2}^{\mathrm{RR}, \mathrm{A}_{1}}\right] J_{m+1}-\left[\mathrm{d} \sigma_{m+1}^{\mathrm{RV}, \mathrm{A}_{1}}+\left(\int_{1} \mathrm{~d} \sigma_{m+2}^{\mathrm{RR}, \mathrm{A}_{1}}\right)^{\mathrm{A}_{1}}\right] J_{m}\right\}_{\epsilon=0} \\
& \mathrm{~d} \sigma_{m}^{\mathrm{NNLO}}=\left\{\mathrm{d} \sigma_{m}^{\mathrm{VV}}+\int_{2}\left[\mathrm{~d} \sigma_{m+2}^{\mathrm{RR}, \mathrm{A}_{2}}-\mathrm{d} \sigma_{m+2}^{\mathrm{RR}, \mathrm{A}_{12}}\right]+\int_{1}\left[\mathrm{~d} \sigma_{m+1}^{\mathrm{RV}, \mathrm{A}_{1}}+\left(\int_{1} \mathrm{~d} \sigma_{m+2}^{\mathrm{RR}, \mathrm{A}_{1}}\right)^{\mathrm{A}_{1}}\right]\right\}_{\epsilon=0} J_{m}
\end{aligned}
$$

is integrable in four dimensions by construction [20, 22, 23].

The counterterms which contribute to $\mathrm{d} \sigma_{m+2}^{\mathrm{NNLO}}$ and to $\mathrm{d} \sigma_{m+1}^{\mathrm{NNO}}$ were introduced in refs. [22] and [23], respectively. The integral of the real-virtual counterterms (the last two terms of eq. (1.10)) was performed in refs. [25-27]. The integral of the iterated singly unresolved counterterm (the third term of eq. (1.10)) was performed in ref. [31]. In this paper, we compute the integral of the collinear-type contributions to the doubly unresolved counterterm (the second term of eq. (1.10)). The soft-type contributions to the same integral are presented in a companion paper [51].

\section{Notation}

In this paper, we use the notation introduced in ref. [31], which we recall in this section. 


\subsection{Matrix elements}

We consider processes with coloured particles (partons) in the final state, while the initialstate particles are colourless (typically electron-positron annihilation into hadrons). Any number of additional non-coloured final-state particles are allowed, too, but they will be suppressed in the notation. Resolved partons in the final state are labeled with letters chosen from the middle of the alphabet, $i, j, k, l, \ldots$, while letters $r, s$ denote unresolved final-state partons.

We adopt the colour- and spin-state notation of ref. [2]. In that notation the amplitude $|\mathcal{M}\rangle$ for a scattering process is an abstract vector in colour and spin space, and its normalisation is fixed such that the squared amplitude summed over colours and spins is

$$
|\mathcal{M}|^{2}=\langle\mathcal{M}|| \mathcal{M}\rangle \text {. }
$$

In this paper we only need $|\mathcal{M}\rangle$ in the Born approximation, denoted by $\left|\mathcal{M}^{(0)}\right\rangle$.

Using the colour-state notation, we define the two-parton colour-correlated squared tree amplitudes as

$$
\left|\mathcal{M}_{(i, k)}^{(0)}\right|^{2} \equiv\left|\mathcal{M}^{(0)}\right|^{2} \otimes \boldsymbol{T}_{i} \boldsymbol{T}_{k} \equiv\left\langle\mathcal{M}^{(0)}\left|\boldsymbol{T}_{i} \cdot \boldsymbol{T}_{k}\right| \mathcal{M}^{(0)}\right\rangle
$$

and similarly the four-parton colour-correlated squared tree amplitudes,

$$
\left|\mathcal{M}_{(i, k),(j, l)}^{(0)}\right|^{2} \equiv\left|\mathcal{M}^{(0)}\right|^{2} \otimes\left\{\boldsymbol{T}_{i} \cdot \boldsymbol{T}_{k}, \boldsymbol{T}_{j} \cdot \boldsymbol{T}_{l}\right\} \equiv\left\langle\mathcal{M}^{(0)}\left|\left\{\boldsymbol{T}_{i} \cdot \boldsymbol{T}_{k}, \boldsymbol{T}_{j} \cdot \boldsymbol{T}_{l}\right\}\right| \mathcal{M}^{(0)}\right\rangle,
$$

where we also introduced the $\otimes$ product notation to indicate the insertion of colour charge operators between $\left\langle\mathcal{M}^{(0)}\right|$ and $\left|\mathcal{M}^{(0)}\right\rangle$. The colour-charge algebra for the product $\sum_{n}\left(\boldsymbol{T}_{i}\right)^{n}\left(\boldsymbol{T}_{k}\right)^{n} \equiv \boldsymbol{T}_{i} \cdot \boldsymbol{T}_{k}$ is:

$$
\boldsymbol{T}_{i} \cdot \boldsymbol{T}_{k}=\boldsymbol{T}_{k} \cdot \boldsymbol{T}_{i} \quad \text { if } \quad i \neq k ; \quad \boldsymbol{T}_{i}^{2}=C_{f_{i}} .
$$

Here $C_{f_{i}}$ is the quadratic Casimir operator in the representation of particle $i$ and we have $C_{q} \equiv C_{\mathrm{F}}=T_{\mathrm{R}}\left(N_{\mathrm{c}}^{2}-1\right) / N_{\mathrm{c}}=\left(N_{\mathrm{c}}^{2}-1\right) /\left(2 N_{\mathrm{c}}\right)$ in the fundamental and $C_{g} \equiv C_{\mathrm{A}}=2 T_{\mathrm{R}} N_{\mathrm{c}}=$ $N_{\mathrm{c}}$ in the adjoint representation, with $T_{\mathrm{R}}=1 / 2$.

We also use squared colour charges with multiple indices, such as $\boldsymbol{T}_{i r}^{2} \equiv C_{f_{i r}}$ and $\boldsymbol{T}_{\text {irs }}^{2} \equiv C_{f_{i r s}}$. In such cases the multiple index denotes a single parton with flavour obtained using the flavour summation rules: odd/even number of quarks plus any number of gluons gives a quark/gluon, or explicitly for the relevant cases at NNLO,

$$
\begin{aligned}
& q+g=q, \quad q+\bar{q}=g, \quad g+g=g, \\
& q+g+g=q, \quad q+q+\bar{q}=q, \quad g+q+\bar{q}=g, \quad g+g+g=g .
\end{aligned}
$$

\subsection{Cross sections}

In this paper we shall need to use only tree-level $n$-parton production cross sections, with $n=m$, the Born cross section, and $n=m+2$, the so-called doubly real correction. We have

$$
\mathrm{d} \sigma_{n}^{(0)}(\{p\})=\mathcal{N} \sum_{\{n\}} \mathrm{d} \phi_{n}(\{p\}) \frac{1}{S_{\{n\}}}\left|\mathcal{M}_{n}^{(0)}(\{p\})\right|^{2},
$$


where $\mathcal{N}$ includes all QCD-independent factors and $\mathrm{d} \phi_{n}(\{p\})$ is the $d$-dimensional phase space for $n$ outgoing particles with momenta $\{p\} \equiv\left\{p_{1}, \ldots, p_{n}\right\}$ and total incoming momentum $Q$,

$$
\mathrm{d} \phi_{n}\left(p_{1}, \ldots, p_{n} ; Q\right)=\prod_{i=1}^{n} \frac{\mathrm{d}^{d} p_{i}}{(2 \pi)^{d-1}} \delta_{+}\left(p_{i}^{2}\right)(2 \pi)^{d} \delta^{(d)}\left(Q-\sum_{i=1}^{n} p_{i}\right) .
$$

The symbol $\sum_{\{n\}}$ denotes summation over different subprocesses and $S_{\{n\}}$ is the Bose symmetry factor for identical particles in the final state. Then the Born cross section and the doubly real correction are simply

$$
\mathrm{d} \sigma_{m}^{\mathrm{B}}(\{p\}) \equiv \mathrm{d} \sigma_{m}^{(0)}(\{p\}) \quad \text { and } \quad \mathrm{d} \sigma_{m+2}^{\mathrm{RR}}(\{p\}) \equiv \mathrm{d} \sigma_{m+2}^{(0)}(\{p\}) .
$$

The final result will also contain the phase-space factor due to the integral over the $(d-3)$-dimensional solid angle, which is included in the definition of the running coupling in the $\overline{\mathrm{MS}}$ renormalisation scheme, ${ }^{1}$

$$
S_{\epsilon}=\int \frac{\mathrm{d}^{(d-3)} \Omega}{(2 \pi)^{d-3}}=\frac{(4 \pi)^{\epsilon}}{\Gamma(1-\epsilon)} .
$$

\subsection{Momentum mappings and phase-space factorisation}

The subtraction terms are written in terms of the momenta obtained by the mappings of ref. [22],

$$
\{p\}_{m+2} \stackrel{\mathrm{X}_{R R}}{\longrightarrow}\{\tilde{p}\}_{m}^{(R R)},
$$

where $\stackrel{\mathrm{X}_{R R}}{\longrightarrow}$ may label a triple collinear, double collinear or soft collinear mapping. ${ }^{2}$ In particular, the soft collinear mapping is obtained by the iterated application of a basic collinear mapping, followed by a basic soft mapping,

$$
\{p\}_{m+2} \stackrel{\mathcal{C}_{i r}}{\longrightarrow}\{\hat{p}\}_{m+1}^{(i r)} \stackrel{\mathcal{S}_{\hat{s}}}{\longrightarrow}\{\tilde{p}\}_{m}^{(\hat{s}, i r)},
$$

while the former two cases do not have such an iterated form. As the above notation suggests, the final set of $m$ momenta are denoted by tildes, while hats indicate the intermediate set of $m+1$ momenta. In kinematic expressions where only the label of a momentum is displayed (we shall discuss several examples below), the tilde is inherited by the label, and we write for instance $\widetilde{i}, \widetilde{i r}$ and $\widetilde{i r s}$, where the last two label single momenta. However, since these mappings affect only the momenta, but not the colour and flavour (apart from the flavour summation rules of eq. (2.5)), we shall omit the tilde from flavour and colour indices.

\footnotetext{
${ }^{1}$ In the $\overline{\mathrm{MS}}$ renormalisation scheme as often employed in the literature, the definition of the running coupling includes the factor $S_{\epsilon}=(4 \pi)^{\epsilon} \mathrm{e}^{-\epsilon \gamma_{E}}$. In a computation at NLO accuracy, the two definitions lead to the same expressions. At NNLO they lead to slightly different bookkeeping of the IR and UV poles at intermediate steps of the computation, our definition leading to somewhat simpler expressions. Of course the physical cross section does not depend on these details.

${ }^{2}$ Integration of the subtraction terms obtained with the double soft mapping are presented in the companion paper [51].
} 
We also use labels such as $(i r)$ to denote a single momentum that is simply the sum of two momenta, $p_{(i r)}^{\mu} \equiv p_{i}^{\mu}+p_{r}^{\mu}$.

The momentum mappings are chosen as to lead to an exact factorisation of the phase space. For the triple collinear phase-space mapping, we have,

$$
\begin{aligned}
\{p\}_{m+2} & \stackrel{\mathcal{C}_{i r s}}{\longrightarrow}\{\tilde{p}\}_{m}^{(i r s)}: \\
& \mathrm{d} \phi_{m+2}\left(\{p\}_{m+2} ; Q\right)=\mathrm{d} \phi_{m}\left(\{\tilde{p}\}_{m}^{(i r s)}, Q\right)\left[\mathrm{d} p_{2 ; m}^{(i r s)}\left(p_{r}, p_{s}, \widetilde{p}_{i r s} ; Q\right)\right],
\end{aligned}
$$

for the double collinear mapping,

$$
\begin{aligned}
& \{p\}_{m+2} \stackrel{\mathcal{C}_{i r ; j s}}{\longrightarrow}\{\tilde{p}\}_{m}^{(i r ; j s)}: \\
& \quad \mathrm{d} \phi_{m+2}\left(\{p\}_{m+2} ; Q\right)=\mathrm{d} \phi_{m}\left(\{\tilde{p}\}_{m}^{(i r ; j s)}, Q\right)\left[\mathrm{d} p_{2 ; m}^{(i r ; j s)}\left(p_{r}, p_{s}, \widetilde{p}_{i r}, \widetilde{p}_{j s} ; Q\right)\right],
\end{aligned}
$$

while for the iterated collinear and soft mapping appearing in eq. (2.11),

$$
\begin{aligned}
& \{p\}_{m+2} \stackrel{\mathcal{C}_{i r}}{\longrightarrow}\{\hat{p}\}_{m+1}^{(i r)} \stackrel{\mathcal{S}_{\hat{s}}}{\longrightarrow}\{\tilde{p}\}_{m}^{(\hat{s}, i r)}: \\
& \mathrm{d} \phi_{m+2}\left(\{p\}_{m+2} ; Q\right)=\mathrm{d} \phi_{m}\left(\{\tilde{p}\}_{m}^{(\hat{s}, i r)}\right)\left[\mathrm{d} p_{1 ; m+1}^{(i r)}\left(p_{r}, \hat{p}_{i r} ; Q\right)\right]\left[\mathrm{d} p_{1 ; m}^{(\hat{s})}\left(\hat{p}_{s} ; Q\right)\right] .
\end{aligned}
$$

\subsection{Kinematic variables}

The following types of kinematic variables are used to write the doubly unresolved subtraction terms.

- Two-particle invariants, such as

$$
s_{i r}=2 p_{i} \cdot p_{r} \quad \text { or } \quad s_{i Q}=2 p_{i} \cdot Q .
$$

Two-particle invariants scaled with $Q^{2}$ are denoted by $y_{i j}=s_{i j} / Q^{2}$.

- Momentum fractions $z_{i, r}$ for the splittings $\tilde{p}_{i r} \rightarrow p_{i}+p_{r}$ and $z_{i, r s}$ for the splittings $\tilde{p}_{i r s} \rightarrow p_{i}+p_{r}+p_{s}$

$$
z_{i, r}=\frac{y_{i Q}}{y_{i Q}+y_{r Q}} \quad \text { and } \quad z_{i, r s}=\frac{y_{i Q}}{y_{i Q}+y_{r Q}+y_{s Q}}
$$

with $z_{i, r}+z_{r, i}=1$ and $z_{i, r s}+z_{r, s i}+z_{s, i r}=1\left(z_{r, s i}\right.$ and $z_{s, i r}$ are computed by obvious permutations).

- We also use the eikonal factors,

$$
\mathcal{S}_{j k}(s)=\frac{2 s_{j k}}{s_{j s} s_{k s}}
$$

As mentioned above, the sum of two momenta is often abbreviated with the two indices in parentheses e.g., $p_{i}^{\mu}+p_{r}^{\mu}=p_{(i r)}^{\mu}$, which is also used in other occurrences, such as

$$
s_{(i r) k}=s_{i k}+s_{r k}, \quad \mathcal{S}_{(i r) k}(s)=\frac{2\left(s_{i k}+s_{r k}\right)}{\left(s_{i s}+s_{r s}\right) s_{k s}} .
$$


Finally, we express the integrated subtraction terms as functions of the following (combinations of) invariants:

$$
x_{\widetilde{i}}=y_{\widetilde{i} Q} \quad \text { and } \quad Y_{\widetilde{i} \widetilde{j}, Q}=\frac{y_{\widetilde{i} \widetilde{j}}}{y_{\widetilde{i} Q} y_{\widetilde{j} Q}} .
$$

In the rest frame of $Q^{\mu}, y_{\widetilde{i} Q}=2 \tilde{E}_{i} / Q$ is simply the scaled energy of parton $\tilde{i}$, while $Y_{\widetilde{i} \widetilde{j}, Q}$ is related to $\chi_{i j}$, the angle between momenta $\tilde{p}_{i}^{\mu}$ and $\tilde{p}_{j}^{\mu}$, by $\cos \chi_{i j}=1-2 Y_{\widetilde{i} \widetilde{j}, Q}$.

\section{Integrating the doubly unresolved approximate cross section}

\subsection{The integrated approximate cross section and insertion operator}

The doubly unresolved approximate cross section times the jet function is defined as,

$$
\mathrm{d} \sigma_{m+2}^{\mathrm{RR}, \mathrm{A}_{2}} \odot J_{m}=\mathcal{N} \sum_{\{m+2\}} \mathrm{d} \phi_{m+2}(\{p\}) \frac{1}{S_{\{m+2\}}} \mathcal{A}_{2}^{(0)}\left|\mathcal{M}_{m+2}^{(0)}(p)\right|^{2} \odot J_{m}(p),
$$

where the $\odot$ notation reminds us that the set of momenta entering $J_{m}$ is different from term to term,

$$
\begin{aligned}
& \mathcal{A}_{2}^{(0)}\left|\mathcal{M}_{m+2}^{(0)}(p)\right|^{2} \odot J_{m}(p)= \\
& \sum_{r} \sum_{s}\left\{\sum _ { i \neq r , s } \left[\frac{1}{6} \mathcal{C}_{i r s}^{(0,0)}(\{p\}) J_{m}\left(\{\tilde{p}\}_{m}^{(i r s)}\right)+\sum_{j \neq i, r, s} \frac{1}{8} \mathcal{C}_{i r ; j s}^{(0,0)}(\{p\}) J_{m}\left(\{\tilde{p}\}_{m}^{(i r ; j s)}\right)\right.\right. \\
& \quad+\frac{1}{2}\left(\mathcal{S}_{i r ; s}^{(0,0)}(\{p\})-\mathcal{C}_{i r s} \mathcal{C S}_{i r ; s}^{(0,0)}(\{p\})-\sum_{j \neq i, r, s} \mathcal{C}_{i r ; j s} \mathcal{S}_{i r ; s}^{(0,0)}(\{p\})\right) J_{m}\left(\{\tilde{p}\}_{m}^{(\hat{s}, i r)}\right) \\
& \quad+\left(-\mathcal{C S}_{i r ; s} \mathcal{S}_{r s}^{(0,0)}(\{p\})-\frac{1}{2} \mathcal{C}_{i r s} \mathcal{S}_{r s}^{(0,0)}(\{p\})+\mathcal{C}_{i r s} \mathcal{C S}_{i r ; s} \mathcal{S}_{r s}^{(0,0)}(\{p\})\right. \\
&\left.\left.\left.\quad+\sum_{j \neq i, r, s} \frac{1}{2} \mathcal{C}_{i r ; j s} \mathcal{S}_{r s}^{(0,0)}(\{p\})\right) J_{m}\left(\{\tilde{p}\}_{m}^{(r s)}\right)\right]+\frac{1}{2} \mathcal{S}_{r s}^{(0,0)}(\{p\}) J_{m}\left(\{\tilde{p}\}_{m}^{(r s)}\right)\right\}
\end{aligned}
$$

All terms on the right-hand side of eq. (3.2) are defined in ref. [22].

Using that all momentum mappings lead to the exact factorisation of phase space, $\mathrm{d} \phi_{m+2}(\{p\} ; Q)=\mathrm{d} \phi_{m}(\{\tilde{p}\} ; Q)\left[\mathrm{d} p_{2 ; m}\right]$, and that the jet function $J_{m}$ does not depend on the variables of the factorised two-parton measure, $\left[\mathrm{d} p_{2 ; m}\right]$, we can compute the integral of eq. (3.2) over the two-parton factorised phase space independently of $J_{m}$,

$$
\begin{aligned}
\int_{2} \mathrm{~d} \sigma_{m+2}^{\mathrm{RR}, \mathrm{A}_{2}} & =\mathcal{N} \sum_{\{m+2\}} \mathrm{d} \phi_{m}(\{\tilde{p}\}) \frac{1}{S_{\{m+2\}}}\left[\frac{\alpha_{\mathrm{s}}}{2 \pi} S_{\epsilon}\left(\frac{\mu^{2}}{Q^{2}}\right)^{\epsilon}\right]^{2} \\
\times & \sum_{r} \sum_{s}\left\{\sum _ { i \neq r , s } \left[\frac{1}{6}\left[\mathrm{C}_{i r s}^{(0)}\right]_{f_{i} f_{r} f_{s}}\left(\boldsymbol{T}_{i r s}^{2}\right)^{2}+\sum_{j \neq i, r, s} \frac{1}{8}\left[\mathrm{C}_{i r ; j s}^{(0)}\right]_{f_{i} f_{r} ; f_{j} f_{s}} \boldsymbol{T}_{i r}^{2} \boldsymbol{T}_{j s}^{2}\right.\right. \\
& +\frac{1}{2}\left(\sum_{j \neq i, r, s} \sum_{k \neq j}\left[\mathrm{CS}_{i r ; s}^{(0),(j, k)}\right]_{f_{i} f_{r}} \boldsymbol{T}_{i r}^{2} \boldsymbol{T}_{j} \boldsymbol{T}_{k}-\left[\mathrm{C}_{i r s} \mathrm{CS}_{i r ; s}^{(0)}\right]_{f_{i} f_{r}}\left(\boldsymbol{T}_{i r}^{2}\right)^{2}\right.
\end{aligned}
$$




$$
\begin{aligned}
& \left.-\sum_{j \neq i, r, s}\left[\mathrm{C}_{i r ; j s} \mathrm{CS}_{i r ; s}^{(0)}\right]_{f_{i} f_{r}} \boldsymbol{T}_{i r}^{2} \boldsymbol{T}_{j}^{2}\right)-\boldsymbol{T}_{i r}^{2} \sum_{j} \sum_{l \neq j}\left[\mathrm{CS}_{i r ; s} \mathrm{~S}_{r s}^{(0)}\right]^{(j, l)} \boldsymbol{T}_{j} \boldsymbol{T}_{l} \\
& \left.+\left(\left[\mathrm{C}_{i r s} \mathrm{CS}_{i r ; s} \mathrm{~S}_{r s}^{(0)}\right]-\frac{1}{2}\left[\mathrm{C}_{i r s} \mathrm{~S}_{r s}^{(0)}\right]_{f_{r} f_{s}}\right)\left(\boldsymbol{T}_{i r s}^{2}\right)^{2}+\sum_{j \neq i, r, s} \frac{1}{2}\left[\mathrm{C}_{i r ; j s} \mathrm{~S}_{r s}^{(0)}\right] \boldsymbol{T}_{i r}^{2} \boldsymbol{T}_{j s}^{2}\right] \\
& \left.+\frac{1}{2} \sum_{i, k}\left(\sum_{j, l}\left[\mathrm{~S}_{r s}^{(0)}\right]^{(i, k)(j, l)}\left\{\boldsymbol{T}_{i} \boldsymbol{T}_{k}, \boldsymbol{T}_{j} \boldsymbol{T}_{l}\right\}+\left[\mathrm{S}_{r s}^{(0)}\right]_{f_{r} f_{s}}^{(i, k)} C_{\mathrm{A}} \boldsymbol{T}_{i} \boldsymbol{T}_{k}\right)\right\} \otimes\left|\mathcal{M}_{m}^{(0)}(\{\tilde{p}\})\right|^{2} .
\end{aligned}
$$

In order to make the integrated subtraction terms dimensionless in color space, we have factored out quadratic Casimir operators in eq. (3.3).

Eq. (3.3) is not yet in the form of an $m$-parton contribution times a factor. In order to obtain such a form, we must still perform summation over unresolved flavours, rewriting the symmetry factor of an $m+2$-parton configuration to the symmetry factor of an $m$ parton configuration. The counting is performed as in the appendix of ref. [31]. As a result of the summation over the unresolved flavours, we obtain functions - the flavour-summed integrated counterterms - denoted by $\left(X^{(0)}\right)_{f_{i} \ldots}^{(j, l) \ldots}$, which are specific sums of the non flavour-summed integrated subtraction terms, $\left[X^{(0)}\right]_{f_{k} \ldots}^{(j, l) \ldots}$. Schematically, we may write,

$$
\left(X^{(0)}\right)_{f_{i} \ldots}^{(j, l) \ldots}=\sum\left[X^{(0)}\right]_{f_{k} \ldots}^{(j, l) \ldots}
$$

The flavour indices on the left- and right-hand sides of this equation need not match: the non flavour-summed functions on the right-hand side carry dependence on unresolved flavours, while the flavour-summed functions on the left do not, by definition.

After summing over unobserved flavours, the integrated doubly unresolved approximate cross section can be written as,

$$
\int_{2} \mathrm{~d} \sigma_{m+2}^{\mathrm{RR}, \mathrm{A}_{2}}=\mathrm{d} \sigma_{m}^{\mathrm{B}} \otimes \boldsymbol{I}_{2}^{(0)}\left(\{p\}_{m} ; \epsilon\right)
$$

where the insertion operator (in colour space) has three contributions according to the possible colour structures,

$$
\begin{aligned}
\boldsymbol{I}_{2}^{(0)}\left(\{p\}_{m} ; \epsilon\right)=\left[\frac{\alpha_{\mathrm{s}}}{2 \pi} S_{\epsilon}\left(\frac{\mu^{2}}{Q^{2}}\right)^{\epsilon}\right]^{2}\{ & \sum_{i}\left[\mathrm{C}_{2, i}^{(0)} \boldsymbol{T}_{i}^{2}+\sum_{j \neq i} \mathrm{C}_{2, i j}^{(0)} \boldsymbol{T}_{j}^{2}\right] \boldsymbol{T}_{i}^{2} \\
& +\sum_{l} \sum_{j \neq l}\left[\mathrm{~S}_{2}^{(0),(j, l)} C_{\mathrm{A}}+\sum_{i} \mathrm{CS}_{2, i}^{(0),(j, l)} \boldsymbol{T}_{i}^{2}\right] \boldsymbol{T}_{j} \boldsymbol{T}_{l} \\
& \left.+\sum_{i, j, k, l} \mathrm{~S}_{2}^{(0),(i, k)(j, l)}\left\{\boldsymbol{T}_{i} \boldsymbol{T}_{k}, \boldsymbol{T}_{j} \boldsymbol{T}_{l}\right\}\right\}
\end{aligned}
$$

with $\boldsymbol{T}_{i}^{2}=C_{f_{i}}\left(C_{q}=C_{\mathrm{F}}, C_{g}=C_{\mathrm{A}}\right)$ as in eq. (2.4). 
In terms of flavour-summed integrated counterterms discussed above, the kinematic functions of the insertion operator can be written as,

$$
\begin{aligned}
\mathrm{C}_{2, i}^{(0)} & =\left(\mathrm{C}_{i r s}^{(0)}\right)_{f_{i}}-\left(\mathrm{C}_{i r s} \mathrm{CS}_{i r ; s}^{(0)}\right)_{f_{i}}-\left(\mathrm{C}_{i r s} \mathrm{~S}_{r s}^{(0)}\right)_{f_{i}}+\left(\mathrm{C}_{i r s} \mathrm{CS}_{i r ; s} \mathrm{~S}_{r s}^{(0)}\right)_{f_{i}}, \\
\mathrm{C}_{2, i j}^{(0)} & =\left(\mathrm{C}_{i r ; j s}^{(0)}\right)_{f_{i} f_{j}}-\left(\mathrm{C}_{i r ; j s} \mathrm{CS}_{i r ; s}^{(0)}\right)_{f_{i} f_{j}}+\left(\mathrm{C}_{i r ; j s} \mathrm{~S}_{r s}^{(0)}\right)_{f_{i} f_{j}}, \\
\mathrm{CS}_{2, i}^{(0),(j, l)} & =\left(\mathrm{CS}_{i r ; s}^{(0)}\right)_{f_{i}}^{(j, l)}-\left(\mathrm{CS}_{i r ; s} \mathrm{~S}_{r s}^{(0)}\right)_{f_{i}}^{(j, l)}, \\
\mathrm{S}_{2}^{(0),(j, l)} & =\left(\mathrm{S}_{r s}^{(0)}\right)^{(j, l)} \\
\mathrm{S}_{2}^{(0),(i, k)(j, l)} & =\left(\mathrm{S}_{r s}^{(0)}\right)^{(i, k)(j, l)} .
\end{aligned}
$$

On the right-hand side of eq. (3.7), the flavour-summed functions depend on the kinematics through variables of the type $x_{i}$ and $Y_{i j, Q}$. The latter dependence stems from integrating an eikonal factor which is always multiplied by a colour-connected squared matrix element. In order to make the results more transparent, we hid the arguments of the functions, but kept the relation to the colour-connected matrix elements, shown as upper indices.

\subsection{Flavour-summed integrated counterterms}

On the right-hand side of eq. (3.7), the flavour-summed functions are the following combinations of the integrated subtraction terms,

1. Triple collinear:

$$
\begin{aligned}
\left(\mathrm{C}_{\text {irs }}^{(0)}\right)_{q} & =\frac{1}{2}\left[\mathrm{C}_{i r s}^{(0)}\right]_{q g g}+\left(n_{\mathrm{f}}-1\right)\left[\mathrm{C}_{i r s}^{(0)}\right]_{q q^{\prime} \bar{q}^{\prime}}+\frac{1}{2}\left[\mathrm{C}_{i r s}^{(0)}\right]_{q q \bar{q}}, \quad q^{\prime} \neq q, \\
\left(\mathrm{C}_{\text {irs }}^{(0)}\right)_{g} & =\frac{1}{6}\left[\mathrm{C}_{\text {irs }}^{(0)}\right]_{g g g}+n_{\mathrm{f}}\left[\mathrm{C}_{i r s}^{(0)}\right]_{g q \bar{q}} .
\end{aligned}
$$

The factor $\left(n_{\mathrm{f}}-1\right)$, which appears after summing over the unresolved flavours, can be traded for $n_{\mathrm{f}}$ by introducing the integrated subtraction term $\left[\mathrm{C}_{i r s}^{(0)}\right]_{q \bar{q} q}^{(\mathrm{id})}$, defined by

$$
\left[\mathrm{C}_{i r s}^{(0)}\right]_{q \bar{q} q}=2\left[\mathrm{C}_{i r s}^{(0)}\right]_{q \bar{q}^{\prime} q^{\prime}}+\left[\mathrm{C}_{i r s}^{(0)}\right]_{q \bar{q} q}^{(\mathrm{id})} .
$$

This definition matches the decomposition of the same-flavour triple splitting function computed in ref. [52]. Thus, we obtain

$$
\left(\mathrm{C}_{i r s}^{(0)}\right)_{q}=\frac{1}{2}\left[\mathrm{C}_{i r s}^{(0)}\right]_{q g g}+n_{\mathrm{f}}\left[\mathrm{C}_{i r s}^{(0)}\right]_{q \bar{q}^{\prime} q^{\prime}}+\frac{1}{2}\left[\mathrm{C}_{i r s}^{(0)}\right]_{q \bar{q} q}^{(\mathrm{id})}, \quad q^{\prime} \neq q .
$$

2. Triple collinear — soft collinear:

$$
\begin{aligned}
& \left(\mathrm{C}_{i r s} \mathrm{CS}_{i r ; s}^{(0)}\right)_{q}=\left[\mathrm{C}_{i r s} \mathrm{CS}_{i r ; s}^{(0)}\right]_{q g} \\
& \left(\mathrm{C}_{i r s} \mathrm{CS}_{i r ; s}^{(0)}\right)_{g}=\frac{1}{2}\left[\mathrm{C}_{i r s} \mathrm{CS}_{i r ; s}^{(0)}\right]_{g g}+n_{\mathrm{f}}\left[\mathrm{C}_{i r s} \mathrm{CS}_{i r ; s}^{(0)}\right]_{q \bar{q}}
\end{aligned}
$$


3. Double collinear:

$$
\begin{aligned}
\left(\mathrm{C}_{i r ; j s}^{(0)}\right)_{q q} & =\frac{1}{2}\left[\mathrm{C}_{i r ; j s}^{(0)}\right]_{q g ; q g}, \\
\left(\mathrm{C}_{i r ; j s}^{(0)}\right)_{q g} & =\frac{1}{4}\left[\mathrm{C}_{i r ; j s}^{(0)}\right]_{q g ; g g}+\frac{n_{\mathrm{f}}}{2}\left[\mathrm{C}_{i r ; j s}^{(0)}\right]_{q g ; q \bar{q}}, \\
\left(\mathrm{C}_{i r ; j s}^{(0)}\right)_{g q} & =\frac{1}{4}\left[\mathrm{C}_{i r ; j s}^{(0)}\right]_{g g ; q g}+\frac{n_{\mathrm{f}}}{2}\left[\mathrm{C}_{i r ; j s}^{(0)}\right]_{q \bar{q} ; q g}, \\
\left(\mathrm{C}_{i r ; j s}^{(0)}\right)_{g g} & =\frac{1}{8}\left[\mathrm{C}_{i r ; j s}^{(0)}\right]_{g g ; g g}+\frac{n_{\mathrm{f}}^{2}}{2}\left[\mathrm{C}_{i r ; j s}^{(0)}\right]_{q \bar{q} ; q^{\prime} \bar{q}^{\prime}} \\
& +\frac{n_{\mathrm{f}}}{4}\left\{\left[\mathrm{C}_{i r ; j s}^{(0)}\right]_{g g ; q \bar{q}}+\left[\mathrm{C}_{i r ; j s}^{(0)}\right]_{q \bar{q} ; g g}\right\}, \quad \forall q, q^{\prime} .
\end{aligned}
$$

4. Double collinear — soft collinear:

$$
\begin{aligned}
&\left(\mathrm{C}_{i r ; j s} \mathrm{CS}_{i r ; s}^{(0)}\right)_{q f}=\left[\mathrm{C}_{i r ; j s} \mathrm{CS}_{i r ; s}^{(0)}\right]_{q g}, \\
&\left(\mathrm{C}_{i r ; j s} \mathrm{CS}_{i r ; s}^{(0)}\right)_{g f}=\frac{1}{2}\left[\mathrm{C}_{i r ; j s} \mathrm{CS}_{i r ; s}^{(0)}\right]_{g g}+n_{\mathrm{f}}\left[\mathrm{C}_{i r ; j s} \mathrm{CS}_{i r ; s}^{(0)}\right]_{q \bar{q}},
\end{aligned}
$$

i.e., it is independent of the flavour $f$.

5. Soft collinear:

$$
\begin{aligned}
& \left(\mathrm{CS}_{i r ; s}^{(0)}\right)_{q}^{(j, l)}=\left[\mathrm{CS}_{i r ; s}^{(0)}\right]_{q g}^{(j, l)} \\
& \left(\mathrm{CS}_{i r ; s}^{(0)}\right)_{g}^{(j, l)}=\frac{1}{2}\left[\mathrm{CS}_{i r ; s}^{(0)}\right]_{g g}^{(j, l)}+n_{\mathrm{f}}\left[\mathrm{CS}_{i r ; s}^{(0)}\right]_{q \bar{q}}^{(j, l)} .
\end{aligned}
$$

6. Triple collinear — double soft:

$$
\left(\mathrm{C}_{i r s} \mathrm{~S}_{r s}^{(0)}\right)_{f}=\frac{1}{2}\left[\mathrm{C}_{i r s} \mathrm{~S}_{r s}^{(0)}\right]_{f g g}+n_{\mathrm{f}}\left[\mathrm{C}_{i r s} \mathrm{~S}_{r s}^{(0)}\right]_{f \bar{q} q} .
$$

7. Triple collinear — soft collinear — double soft:

$$
\left(\mathrm{C}_{i r s} \mathrm{CS}_{i r ; s} \mathrm{~S}_{r s}^{(0)}\right)_{f}=\left[\mathrm{C}_{i r s} \mathrm{CS}_{i r ; s} \mathrm{~S}_{r s}^{(0)}\right],
$$

i.e., it is independent of the flavour $f$.

8. Double collinear — double soft:

$$
\left(\mathrm{C}_{i r ; j s} \mathrm{~S}_{r s}^{(0)}\right)_{f_{1} f_{2}}=\frac{1}{2}\left[\mathrm{C}_{i r ; j s} \mathrm{~S}_{r s}^{(0)}\right],
$$

i.e., it is independent of the flavours $f_{1}$ and $f_{2}$.

9. Double soft:

$$
\begin{aligned}
\left(\mathrm{S}_{r s}^{(0)}\right)^{(i, k)(j, l)} & =\frac{1}{2}\left[\mathrm{~S}_{r s}^{(0)}\right]^{(i, k)(j, l)}, \\
\left(\mathrm{S}_{r s}^{(0)}\right)^{(j, l)} & =\frac{1}{2}\left[\mathrm{~S}_{r s}^{(0)}\right]_{g g}^{(j, l)}+n_{\mathrm{f}}\left[\mathrm{S}_{r s}^{(0)}\right]_{\bar{q} q}^{(j, l)} .
\end{aligned}
$$


10. Soft collinear — double soft:

$$
\left(\mathrm{CS}_{i r ; s} \mathrm{~S}_{r s}^{(0)}\right)_{f}^{(j, l)}=\left[\mathrm{CS}_{i r ; s} \mathrm{~S}_{r s}^{(0)}\right]^{(j, l)},
$$

i.e., it is independent of the flavour $f$.

In this paper, we compute the collinear-type counterterms, which do not involve $\mathrm{S}_{r s}^{(0)}$, i.e., those in eqs. (3.10)-(3.14). The integration of the subtraction terms which are obtained by the double soft mapping is presented in a companion paper [51].

\section{Integrated counterterms}

In this section we define the integrated counterterms and compute them in terms of master integrals.

\subsection{Integrated triple collinear counterterm}

The integral of the triple collinear counterterm is

$$
\begin{aligned}
{\left[\mathrm{C}_{i r s}^{(0)}\right]_{f_{i} f_{r} f_{s}}=} & \left(\frac{(4 \pi)^{2}}{S_{\epsilon}} Q^{2 \epsilon}\right)^{2} \int_{2}\left[\mathrm{~d} p_{2 ; m}^{(i r s)}\left(p_{r}, p_{s}, \tilde{p}_{i r s} ; Q\right)\right] \frac{1}{s_{i r s}^{2}} \frac{1}{\left(\boldsymbol{T}_{i r s}^{2}\right)^{2}} \\
& \times P_{f_{i} f_{r} f_{s}}^{(0)}\left(z_{i, r s}, z_{r, i s}, z_{s, i r}, s_{i r}, s_{i s}, s_{r s} ; \epsilon\right) f\left(\alpha_{0}, \alpha_{i r s}, d(m, \epsilon)\right) .
\end{aligned}
$$

Here $P_{f_{i} f_{r} f_{s}}^{(0)}\left(z_{i, r s}, z_{r, i s}, z_{s, i r}, s_{i r}, s_{i s}, s_{r s} ; \epsilon\right)$ are the complete ${ }^{3}$ spin-averaged three-parton splitting functions, recalled in appendix B, where we explain why some of these are different from those presented in ref. [52]. For consistency of the complete subtraction scheme one must use the forms given in appendix B. The function $f\left(\alpha_{0}, \alpha_{i r s}, d(m, \epsilon)\right)$ is defined and its role is explained in appendix A. The other subtraction terms discussed in this paper will also contain such harmless modifications as compared to the original ones in ref. [22].

Following the decomposition of the triple collinear splitting functions into abelian and non-abelian pieces [52], we also decompose the integrated functions $\left[\mathrm{C}_{i r s}^{(0)}\right]_{\text {ggg }}$ and $\left[\mathrm{C}_{\text {irs }}^{(0)}\right]_{g q \bar{q}}$ likewise,

$$
\left[\mathrm{C}_{i r s}^{(0)}\right]_{q g g}=\left[\mathrm{C}_{i r s}^{(0)}\right]_{q g g}^{(\mathrm{ab})}+\left[\mathrm{C}_{i r s}^{(0)}\right]_{q g g}^{(\mathrm{nab})} \quad \text { and } \quad\left[\mathrm{C}_{i r s}^{(0)}\right]_{g q \bar{q}}=\left[\mathrm{C}_{i r s}^{(0)}\right]_{g q \bar{q}}^{(\mathrm{ab})}+\left[\mathrm{C}_{i r s}^{(0)}\right]_{g q \bar{q}}^{(\mathrm{nab})} .
$$

They are computed in appendix C. The result can be written as

$$
\left[\mathrm{C}_{i r s}^{(0)}\right]_{f_{i} f_{r} f_{s}}=a_{f_{i} f_{r} f_{s}} \sum_{n=1}^{5} \sum_{j, k, l, m} c_{f_{i} f_{r} f_{s}, n}^{(0), j, k, l, m} \mathcal{I}_{2 \mathcal{C}, n}^{(j, k, l, m)}\left(x_{\overparen{i r s}}, \epsilon ; \alpha_{0}, d_{0}\right),
$$

where the constants $a_{f_{i} f_{r} f_{s}}$ denote colour-factor ratios as follows:

$$
\begin{aligned}
& a_{q \bar{q}^{\prime} q^{\prime}}=\frac{T_{\mathrm{R}}}{C_{\mathrm{F}}} \\
& a_{q \bar{q} q}^{(\mathrm{id})}=1-\frac{C_{\mathrm{A}}}{2 C_{\mathrm{F}}} \\
& a_{q g g}^{(\mathrm{ab})}=1, \\
& a_{q g g}^{(\mathrm{nab})}=\frac{C_{\mathrm{A}}}{C_{\mathrm{F}}}, \\
& a_{g q \bar{q}}^{(\mathrm{ab})}=\frac{C_{\mathrm{F}} T_{\mathrm{R}}}{C_{\mathrm{A}}^{2}}, \\
& a_{g q \bar{q}}^{(\mathrm{nab})}=\frac{T_{\mathrm{R}}}{C_{\mathrm{A}}}, \\
& a_{g g g}=1 \text {. }
\end{aligned}
$$

The non-zero coefficients are listed in tables $1-5$, while the integrals $\mathcal{I}_{2 \mathcal{C}, n}^{(j, k, l, m)}\left(x, \epsilon ; \alpha_{0}, d_{0}\right)$ $(n=1, \ldots, 5)$ are defined and computed in appendix C.

\footnotetext{
${ }^{3}$ As opposed to abelian and non-abelian parts, see appendix B.2.
} 


\begin{tabular}{|c|c|c|c|c|c|c|c|c|c|}
\hline$j \quad k$ & $k \quad l$ & $m$ & $c_{q \bar{q}^{\prime} q^{\prime} ; 1}^{(0)}$ & $c_{q \bar{q} q ; 1}^{(0),(\mathrm{id})}$ & $c_{q g g ; 1}^{(0),(\mathrm{ab})}$ & $c_{q g g ; 1}^{(0),(\mathrm{nab})}$ & $c_{g q \bar{q} ; 1}^{(0),(\mathrm{ab})}$ & $c_{g q \bar{q} ; 1}^{(0),(\mathrm{nab})}$ & $c_{g g g ; 1}^{(0)}$ \\
\hline 0 & $\begin{array}{ll}0 & 0\end{array}$ & 00 & $-1+\epsilon$ & $-4+2 \epsilon+2 \epsilon^{2}$ & $2(1-\epsilon) \epsilon$ & $1-2 \epsilon+\epsilon^{2}$ & $-2 \epsilon$ & $-1+\epsilon$ & $6-6 \epsilon$ \\
\hline $0-$ & -10 & $0 \quad 0$ & 0 & $6-10 \epsilon-2 \epsilon^{2}$ & $-2(1-2 \epsilon) \epsilon$ & $\frac{3}{2}+\frac{15 \epsilon}{2}-\epsilon^{2}$ & -4 & $\frac{4}{1-\epsilon}$ & $18+6 \epsilon$ \\
\hline $0-$ & -10 & 01 & 0 & $-4+4 \epsilon+2 \epsilon^{2}$ & $2(1-\epsilon) \epsilon$ & $\frac{5}{2}-\frac{9 \epsilon}{2}+\epsilon^{2}$ & 0 & $-\frac{4}{1-\epsilon}$ & 0 \\
\hline $1-$ & -10 & 00 & -2 & $-4+4 \epsilon$ & $-2+4 \epsilon-2 \epsilon^{2}$ & $2-2 \epsilon$ & $2-2 \epsilon$ & -2 & $6-6 \epsilon$ \\
\hline-1 & $\begin{array}{ll}0 & 0\end{array}$ & 01 & $-2 \epsilon$ & $-2+6 \epsilon$ & $2(1-\epsilon) \epsilon$ & $2-4 \epsilon$ & $4 \frac{1+\epsilon}{1-\epsilon}$ & $-4 \frac{2+\epsilon}{1-\epsilon}$ & -12 \\
\hline $0-$ & $-2 \quad 0$ & 00 & -2 & 0 & 0 & $2-2 \epsilon$ & 0 & -2 & $6-6 \epsilon$ \\
\hline $1-$ & -20 & $0 \quad 0$ & 4 & 0 & 0 & $-4+4 \epsilon$ & 0 & 4 & $-12+12 \epsilon$ \\
\hline $2-$ & -20 & $0 \quad 0$ & -2 & 0 & 0 & $2-2 \epsilon$ & 0 & -2 & $6-6 \epsilon$ \\
\hline$-1-$ & -10 & $0 \quad 0$ & 0 & $2 \epsilon$ & 6 & $-5+2 \epsilon$ & $-\frac{2 \epsilon}{1-\epsilon}$ & $-\frac{7-4 \epsilon}{1-\epsilon}$ & 51 \\
\hline$-1-$ & -10 & 01 & 0 & $6-2 \epsilon$ & $-6+2 \epsilon$ & $7-5 \epsilon$ & 0 & $2 \frac{7-\epsilon}{1-\epsilon}$ & -84 \\
\hline$-1-$ & $\begin{array}{ll}-1 & 0\end{array}$ & 02 & 0 & 0 & 0 & 0 & $\frac{4}{1-\epsilon}$ & $-\frac{6}{1-\epsilon}$ & 6 \\
\hline-1 & $0-1$ & 11 & 0 & $\epsilon(1+\epsilon)$ & $-\epsilon(1+\epsilon)$ & $2-\frac{3 \epsilon}{2}+\frac{\epsilon^{2}}{2}$ & $\frac{2}{1-\epsilon}$ & $\frac{4-\epsilon}{1-\epsilon}$ & -39 \\
\hline-1 & $0-1$ & 12 & 0 & 0 & 0 & 0 & $-\frac{2 \epsilon}{1-\epsilon}$ & $-\frac{3-2 \epsilon}{1-\epsilon}$ & 9 \\
\hline
\end{tabular}

Table 1. Non-zero coefficients of the $\mathcal{I}_{2 \mathcal{C}, 1}^{(j, k, l, m)}\left(x, \epsilon ; \alpha_{0}, d_{0}\right)$ integrals.

\subsection{Integrated double collinear counterterm}

The integral of the double collinear counterterm is

$$
\begin{aligned}
{\left[\mathrm{C}_{i r ; j s}^{(0)}\right]_{f_{i} f_{r} ; f_{j} f_{s}}=} & \left(\frac{(4 \pi)^{2}}{S_{\epsilon}} Q^{2 \epsilon}\right)^{2} \int\left[\mathrm{d} p_{2 ; m}^{(i r ; j s)}\left(p_{r}, p_{s}, \tilde{p}_{i r}, \tilde{p}_{j s} ; Q\right)\right] \frac{1}{s_{i r} s_{j s}} \frac{1}{\boldsymbol{T}_{i r}^{2} \boldsymbol{T}_{j s}^{2}} \\
& \times P_{f_{i} f_{r}}^{(0)}\left(z_{r, i} ; \epsilon\right) P_{f_{j} f_{s}}^{(0)}\left(z_{s, j} ; \epsilon\right) f\left(\alpha_{0}, \alpha_{i r}+\alpha_{j s}, d(m, \epsilon)\right) .
\end{aligned}
$$

In eq. (4.5), $P_{f_{i} f_{r}}^{(0)}\left(z_{r, i} ; \epsilon\right)$ and $P_{f_{i} f_{r}}^{(0)}\left(z_{r, i} ; \epsilon\right)$ are the spin-averaged Altarelli-Parisi splitting functions in $d$ dimensions, as recalled in appendix B.

The integrated counterterm is computed in appendix $\mathrm{D}$. The result is

$$
\left[\mathrm{C}_{i r ; j s}^{(0)}\right]_{f_{i} f_{r} ; f_{j} f_{s}}=a_{f_{i} f_{r}} a_{f_{j} f_{s}} \sum_{k, l=-1}^{2} c_{f_{i} f_{r}}^{(0), k} c_{f_{j} f_{s}}^{(0), l} \mathcal{I}_{2 \mathcal{C}, 6}^{(k, l)}\left(x_{\widetilde{i r}}, x_{\widetilde{j s}} ; \epsilon, \alpha_{0}, d_{0}\right),
$$

where the constants $a_{f_{i} f_{r}}$ denote the colour factor ratios

$$
a_{q \bar{q}}=\frac{T_{\mathrm{R}}}{C_{\mathrm{A}}}, \quad a_{q g}=a_{g g}=1,
$$

and the coefficients $c_{f_{i} f_{r}}$ are those already defined in ref. [27] stripped of their colour factors, as presented in table 6 . 


\begin{tabular}{|c|c|c|c|c|c|c|c|c|c|c|}
\hline$j$ & $k$ & $l$ & $m$ & $c_{q \bar{q}^{\prime} q^{\prime} ; 2}^{(0)}$ & $c_{q \bar{q} q ; 2}^{(0),(\mathrm{id})}$ & $c_{q g g ; 2}^{(0),(\mathrm{ab})}$ & $c_{q g g ; 2}^{(0),(\mathrm{nab})}$ & $c_{g q \bar{q} ; 2}^{(0),(\mathrm{ab})}$ & $c_{g q \bar{q} ; 2}^{(0),(\mathrm{nab})}$ & $c_{g g g ; 2}^{(0)}$ \\
\hline-1 & 0 & -1 & 0 & 0 & 0 & -8 & $5-\epsilon$ & 0 & 1 & -12 \\
\hline-1 & 0 & -1 & 1 & 0 & 0 & $6-2 \epsilon$ & $-3+3 \epsilon$ & 0 & $-\frac{3-\epsilon}{1-\epsilon}$ & 18 \\
\hline-1 & 0 & -1 & 2 & 0 & 0 & $-2+2 \epsilon$ & $2-2 \epsilon$ & 0 & $\frac{4}{1-\epsilon}$ & -12 \\
\hline-1 & 0 & -1 & 3 & 0 & 0 & 0 & 0 & 0 & $-\frac{2}{1-\epsilon}$ & 6 \\
\hline 0 & -1 & -1 & 0 & 0 & 0 & -2 & $-\epsilon$ & 0 & 1 & -12 \\
\hline 0 & -1 & -1 & 1 & 0 & 0 & $-2-2 \epsilon$ & $2 \epsilon$ & 0 & $-\frac{2}{1-\epsilon}$ & 6 \\
\hline 0 & -1 & -1 & 2 & 0 & 0 & 0 & 0 & 0 & $\frac{2}{1-\epsilon}$ & -6 \\
\hline-1 & -1 & -1 & 0 & 0 & 0 & -8 & $5-\epsilon$ & 0 & 1 & -12 \\
\hline-1 & -1 & -1 & 1 & 0 & 0 & $6-2 \epsilon$ & $-3+3 \epsilon$ & 0 & $-\frac{3-\epsilon}{1-\epsilon}$ & 18 \\
\hline-1 & -1 & -1 & 2 & 0 & 0 & $-2+2 \epsilon$ & $2-2 \epsilon$ & 0 & $\frac{4}{1-\epsilon}$ & -12 \\
\hline-1 & -1 & -1 & 3 & 0 & 0 & 0 & 0 & 0 & $-\frac{2}{1-\epsilon}$ & 6 \\
\hline
\end{tabular}

Table 2. Non-zero coefficients of the $\mathcal{I}_{2 \mathcal{C}, 2}^{(j, k, l, m)}\left(x, \epsilon ; \alpha_{0}, d_{0}\right)$ integrals.

\subsection{Integrated soft collinear counterterms}

There are three types of soft collinear counterterms.

1. Soft collinear:

$$
\begin{aligned}
{\left[\mathrm{CS}_{i r ; s}^{(0)}\right]_{f_{i} f_{r}}^{(j, k)}=} & -\left(\frac{(4 \pi)^{2}}{S_{\epsilon}} Q^{2 \epsilon}\right)^{2} \int_{2}\left[\mathrm{~d} p_{1 ; m+1}^{(i r)}\left(p_{r}, \hat{p}_{i r} ; Q\right)\right]\left[\mathrm{d} p_{1 ; m}^{(\hat{s})}\left(\hat{p}_{s} ; Q\right)\right] \\
& \times \frac{1}{2} \mathcal{S}_{j k}(s) \frac{1}{s_{i r}} \frac{1}{\boldsymbol{T}_{i r}^{2}} P_{f_{i} f_{r}}^{(0)}\left(z_{r, i} ; \epsilon\right) f\left(\alpha_{0}, \alpha_{i r}, d(m, \epsilon)\right) f\left(y_{0}, y_{\hat{s} Q}, d^{\prime}(m, \epsilon)\right) .
\end{aligned}
$$

The factor $\mathcal{S}_{j k}(s)$ is the eikonal factor, given by eq. (2.17) if $j, k \neq(i r)$, and by eq. (2.18) if e.g., $j=(i r)$.

The integrated counterterm is computed in appendix E. The result is

$$
\left[\mathrm{CS}_{i r ; s}^{(0)}\right]_{f_{i} f_{r}}^{(j, k)}=a_{f_{i} f_{r}} \sum_{l=-1}^{2} c_{f_{i} f_{r}}^{(0), l} \mathcal{I}_{2 \mathcal{L}, 1}^{(l)}\left(x_{\widetilde{i r}}, Y_{\widetilde{i r} \widetilde{j}, Q}, Y_{\widetilde{i r} \widetilde{k}, Q}, Y_{\widetilde{j} \widetilde{k}, Q} ; \epsilon, \alpha_{0}, y_{0}, d_{0}, d_{0}^{\prime}\right)
$$

if $j, k \neq(i r)$, and

$$
\begin{aligned}
{\left[\mathrm{CS}_{i r ; s}^{(0)}\right]_{f_{i} f_{r}}^{(i r, k)}=a_{f_{i} f_{r}} \sum_{l=-1}^{2} c_{f_{i} f_{r}}^{(0), l} } & \mathcal{I}_{2 \mathcal{C S}, 2}^{(l)}\left(x_{\widetilde{i r}}, Y_{\widetilde{i r} \widetilde{k}, Q} ; \epsilon, \alpha_{0}, y_{0}, d_{0}, d_{0}^{\prime}\right) \\
& \left.+\mathcal{I}_{2 \mathcal{C S}, 3}^{(l)}\left(x_{\widetilde{i r}} ; \epsilon, \alpha_{0}, y_{0}, d_{0}, d_{0}^{\prime}\right)\right]
\end{aligned}
$$




\begin{tabular}{|c|c|c|c|c|c|c|c|c|c|c|}
\hline$j$ & $k$ & $l$ & $m$ & $c_{q \bar{q}^{\prime} q^{\prime} ; 3}^{(0)}$ & $c_{q \bar{q} q ; 3}^{(0),(\mathrm{id})}$ & $c_{q g g ; 3}^{(0),(\mathrm{ab})}$ & $c_{q g g ; 3}^{(0),(\mathrm{nab})}$ & $c_{g q \bar{q} ; 3}^{(0),(\mathrm{ab})}$ & $c_{g q \bar{q} ; 3}^{(0),(\text { nab })}$ & $c_{g g g ; 3}^{(0)}$ \\
\hline 0 & -1 & 0 & -1 & 2 & 0 & 0 & -8 & 0 & 2 & -24 \\
\hline 0 & -1 & 1 & -1 & -2 & $-4+4 \epsilon$ & 0 & $2-2 \epsilon$ & 0 & -2 & $6-6 \epsilon$ \\
\hline 0 & -1 & 2 & -1 & 2 & 0 & 0 & $-2+2 \epsilon$ & 0 & $\frac{8}{1-\epsilon}$ & -24 \\
\hline-1 & 0 & 0 & -1 & 0 & $2-2 \epsilon$ & 0 & -4 & 0 & 1 & -12 \\
\hline-1 & 0 & 1 & -1 & 0 & $4 \epsilon$ & 0 & $3-\epsilon$ & 0 & $-\frac{3-\epsilon}{1-\epsilon}$ & 18 \\
\hline-1 & 0 & 2 & -1 & 0 & $2-2 \epsilon$ & 0 & $-1+\epsilon$ & 0 & $\frac{4}{1-\epsilon}$ & -12 \\
\hline-1 & 0 & 3 & -1 & 0 & 0 & 0 & 0 & 0 & $-\frac{2}{1-\epsilon}$ & 6 \\
\hline 0 & -2 & 1 & -1 & 4 & 0 & 0 & $-4+4 \epsilon$ & 0 & 4 & $-12+12 \epsilon$ \\
\hline 1 & -2 & 1 & -1 & -4 & 0 & 0 & $4-4 \epsilon$ & 0 & -4 & $12-12 \epsilon$ \\
\hline-1 & -1 & 0 & -1 & 0 & -4 & 0 & 2 & 0 & 0 & 0 \\
\hline-1 & -1 & 1 & -1 & 0 & $-2-2 \epsilon$ & 0 & -2 & 0 & 1 & -12 \\
\hline-1 & -1 & 2 & -1 & 0 & $-2+2 \epsilon$ & 0 & $1-\epsilon$ & 0 & $-\frac{2}{1-\epsilon}$ & 6 \\
\hline-1 & -1 & 3 & -1 & 0 & 0 & 0 & 0 & 0 & $\frac{2}{1-\epsilon}$ & -6 \\
\hline 0 & -2 & 2 & -2 & -2 & 0 & 0 & $2-2 \epsilon$ & 0 & -2 & $6-6 \epsilon$ \\
\hline
\end{tabular}

Table 3. Non-zero coefficients of the $\mathcal{I}_{2 \mathcal{C}, 3}^{(j, k, l, m)}\left(x, \epsilon ; \alpha_{0}, d_{0}\right)$ integrals.

\begin{tabular}{|c|c|c|c|c|c|c|c|c|c|c|}
\hline$j$ & $k$ & $l$ & $m$ & $c_{q \bar{q}^{\prime} q^{\prime} ; 4}^{(0)}$ & $c_{q \bar{q} q ; 4}^{(0),(\mathrm{id})}$ & $c_{q g g ; 4}^{(0),(\mathrm{ab})}$ & $c_{q g g ; 4}^{(0),(\mathrm{nab})}$ & $c_{g q \bar{q} ; 4}^{(0),(\mathrm{ab})}$ & $c_{g q \bar{q} ; 4}^{(0),(\mathrm{nab})}$ & $c_{g g g ; 4}^{(0)}$ \\
\hline-1 & 0 & -1 & -1 & 0 & 0 & 4 & 0 & 0 & 0 & 12 \\
\hline 0 & -1 & -1 & -1 & 0 & 0 & 4 & -2 & 0 & 0 & 6 \\
\hline-1 & -1 & -1 & -1 & 0 & 0 & 4 & -2 & 0 & 0 & 6 \\
\hline
\end{tabular}

Table 4. Non-zero coefficients of the $\mathcal{I}_{2 \mathcal{C}, 4}^{(j, k, l, m)}\left(x, \epsilon ; \alpha_{0}, d_{0}\right)$ integrals.

\begin{tabular}{|cccc|c|c|c|c|c|c|c|}
\hline$j$ & $k$ & $l$ & $m$ & $c_{q \bar{q}^{\prime} q^{\prime} ; 5}^{(0)}$ & $c_{q \bar{q} q ; 5}^{(0),(\mathrm{id})}$ & $c_{q g g ; 5}^{(0),(\mathrm{ab})}$ & $c_{q g g ; 5}^{(0),(\mathrm{nab})}$ & $c_{g q \bar{q} ; 5}^{(0),(\mathrm{ab})}$ & $c_{g q \bar{q} ; 5}^{(0),(\mathrm{nab})}$ & $c_{g g g ; 5}^{(0)}$ \\
\hline-1 & -1 & -1 & -1 & 0 & 2 & 0 & 0 & 0 & 0 & 3 \\
\hline
\end{tabular}

Table 5. Non-zero coefficients of the $\mathcal{I}_{2 \mathcal{C}, 5}^{(j, k, l, m)}\left(x, \epsilon ; \alpha_{0}, d_{0}\right)$ integrals. 


\begin{tabular}{|r|c|c|c|}
\hline$k$ & $c_{q g}^{(0), k}$ & $c_{q \bar{q}}^{(0), k}$ & $c_{g g}^{(0), k}$ \\
\hline-1 & 2 & 0 & 4 \\
0 & -2 & 1 & -4 \\
1 & $1-\epsilon$ & $-\frac{2}{1-\epsilon}$ & 2 \\
2 & 0 & $\frac{2}{1-\epsilon}$ & -2 \\
\hline
\end{tabular}

Table 6. Values of the $c_{f_{i} f_{r}}^{(0), k}$ coefficients that appear in eqs. (4.6), (4.9), (4.10), (4.12) and (4.14).

if e.g., $j=(i r)$, where $a_{f_{i} f_{r}}$ and the coefficients are given in eq. (4.7) and table 6 respectively.

2. Triple collinear — soft collinear:

$$
\begin{aligned}
& {\left[\mathrm{C}_{i r s} \mathrm{CS}_{i r ; s}^{(0)}\right]_{f_{i} f_{r}}=\left(\frac{(4 \pi)^{2}}{S_{\epsilon}} Q^{2 \epsilon}\right)^{2} \int_{2}\left[\mathrm{~d} p_{1 ; m+1}^{(i r)}\left(p_{r}, \hat{p}_{i r} ; Q\right)\right]\left[\mathrm{d} p_{1 ; m}^{(\hat{s})}\left(\hat{p}_{s} ; Q\right)\right]} \\
& \quad \times \frac{2}{s_{(i r) s}} \frac{1-z_{s, i r}}{z_{s, i r}} \frac{1}{s_{i r}} \frac{1}{\boldsymbol{T}_{i r}^{2}} P_{f_{i} f_{r}}^{(0)}\left(z_{r, i} ; \epsilon\right) f\left(\alpha_{0}, \alpha_{i r}, d(m, \epsilon)\right) f\left(y_{0}, y_{\hat{s} Q}, d^{\prime}(m, \epsilon)\right),
\end{aligned}
$$

which is computed in appendix E. The result is

$$
\left[\mathrm{C}_{i r s} \mathrm{CS}_{i r ; s}^{(0)}\right]_{f_{i} f_{r}}=a_{f_{i} f_{r}} \sum_{l=-1}^{2} c_{f_{i} f_{r}}^{(0), l} \mathcal{I}_{2 \mathcal{C S}, 4}^{(l)}\left(x_{\widetilde{i r}} ; \epsilon, \alpha_{0}, y_{0}, d_{0}, d_{0}^{\prime}\right) .
$$

3. Double collinear — soft collinear:

$$
\begin{aligned}
{\left[\mathrm{C}_{i r ; j s} \mathrm{CS}_{i r ; s}^{(0)}\right]_{f_{i} f_{r}}=} & \left(\frac{(4 \pi)^{2}}{S_{\epsilon}} Q^{2 \epsilon}\right)^{2} \int_{2}\left[\mathrm{~d} p_{1 ; m+1}^{(i r)}\left(p_{r}, \hat{p}_{i r} ; Q\right)\right]\left[\mathrm{d} p_{1 ; m}^{(\hat{s})}\left(\hat{p}_{s} ; Q\right)\right] \\
& \times \frac{2}{s_{j s}} \frac{z_{j, s}}{z_{s, j}} \frac{1}{s_{i r}} \frac{1}{\boldsymbol{T}_{i r}^{2}} P_{f_{i} f_{r}}^{(0)}\left(z_{r, i} ; \epsilon\right) f\left(\alpha_{0}, \alpha_{i r}, d(m, \epsilon)\right) f\left(y_{0}, y_{\hat{s} Q}, d^{\prime}(m, \epsilon)\right),
\end{aligned}
$$

which is also computed in appendix E, as

$$
\left[\mathrm{C}_{i r ; j s} \mathrm{CS}_{i r ; s}^{(0)}\right]_{f_{i} f_{r}}=a_{f_{i} f_{r}} \sum_{l=-1}^{2} c_{f_{i} f_{r}}^{(0), l} \mathcal{I}_{2 \mathcal{S}, 5}^{(l)}\left(x_{\widetilde{i r}}, Y_{\widetilde{i r} \widetilde{j}, Q} ; \epsilon, \alpha_{0}, y_{0}, d_{0}, d_{0}^{\prime}\right)
$$

\section{Results}

\subsection{Integrated counterterms to $\mathrm{O}\left(\epsilon^{-2}\right)$}

After evaluating the integrals as explained in the appendices, we obtain the kinematic functions defined in eq. (3.7) as Laurent expansions in $\epsilon$. We compute those expansions analytically up to $\mathrm{O}\left(\epsilon^{-2}\right)$, while the remaining coefficients up to $\mathrm{O}\left(\epsilon^{0}\right)$ are calculated numerically via sector decomposition, see [53] and references therein. In obtaining our results, we use 
$d_{0}=D_{0}+d_{1} \epsilon$ and $d_{0}^{\prime}=D_{0}^{\prime}+d_{1}^{\prime} \epsilon$, see eq. (A.9). The parameters $D_{0}, d_{1}, D_{0}^{\prime}$ and $d_{1}^{\prime}$, as well as $\alpha_{0}$ and $y_{0}$ are left symbolic throughout the analytic computation and sector decomposition.

To present analytic results for the non-soft counterterms up to $\mathrm{O}\left(\epsilon^{-2}\right)$, we use the following abbreviations,

$$
\gamma_{q}\left(n_{\mathrm{f}}\right)=\frac{3}{2}, \quad \gamma_{g}\left(n_{\mathrm{f}}\right)=\frac{11}{6}-\frac{2 T_{\mathrm{R}}}{3 C_{\mathrm{A}}} n_{\mathrm{f}} \quad \text { and } \quad \Sigma(z, N)=\ln z-\sum_{i=1}^{N} \frac{1-(1-z)^{k}}{k},
$$

where $\gamma_{q}\left(n_{\mathrm{f}}\right)$ is actually independent of the number of light flavours, but introducing the flavour dependence formally makes possible a flavour-independent notation. Furthermore, the kinematic functions are dimensionless in colour space, hence these $n_{\mathrm{f}}$-dependent $\gamma_{f}\left(n_{\mathrm{f}}\right)$ constants are related to the constans $\gamma_{f}$ often used in the literature [54] by $\gamma_{f} \rightarrow C_{f} \gamma_{f}\left(n_{\mathrm{f}}\right)$.

1. Triple collinear:

$$
\begin{aligned}
\left(\mathrm{C}_{\text {irs }}^{(0)}\right)_{f_{i}}\left(x_{i}\right)= & \frac{1}{2}\left(1+\frac{C_{\mathrm{A}}}{2 C_{f_{i}}}\right)\left[\frac{1}{\epsilon^{4}}-\frac{1}{\epsilon^{3}}\left(4 \ln x_{i}-\gamma_{f_{i}}\left(n_{\mathrm{f}}\right)-\gamma_{f_{i}}\left(\frac{C_{\mathrm{F}}}{C_{f_{i}}} n_{\mathrm{f}}\right)\right)\right] \\
& +\frac{1}{\epsilon^{3}} \frac{C_{\mathrm{A}}}{4 C_{f_{i}}} \gamma_{g}\left(\frac{C_{\mathrm{F}}}{C_{f_{i}}} n_{\mathrm{f}}\right)+\mathrm{O}\left(\epsilon^{-2}\right) .
\end{aligned}
$$

2. Triple collinear — soft collinear:

$$
\begin{aligned}
\left(\mathrm{C}_{i r s} \mathrm{CS}_{i r ; s}^{(0)}\right)_{f_{i}}\left(x_{i}\right)= & \frac{2}{3}\left[\frac{1}{\epsilon^{4}}-\frac{2}{\epsilon^{3}}\left(\ln x_{i}+\Sigma\left(y_{0}, D_{0}^{\prime}-1\right)\right)\right]+\frac{1}{2 \epsilon^{3}} \gamma_{f_{i}}\left(n_{\mathrm{f}}\right) \\
& +\mathrm{O}\left(\epsilon^{-2}\right) .
\end{aligned}
$$

3. Double collinear:

$$
\begin{aligned}
\left(\mathrm{C}_{i r ; j s}^{(0)}\right)_{f_{i} f_{j}}\left(x_{i}, x_{j}\right)= & \frac{1}{2 \epsilon^{4}}-\frac{1}{2 \epsilon^{3}}\left[2\left(\ln x_{i}+\ln x_{j}\right)-\gamma_{f_{i}}\left(n_{\mathrm{f}}\right)-\gamma_{f_{j}}\left(n_{\mathrm{f}}\right)\right] \\
& +\mathrm{O}\left(\epsilon^{-2}\right) .
\end{aligned}
$$

4. Double collinear - soft collinear:

$$
\begin{aligned}
\left(\mathrm{C}_{i r ; j s} \mathrm{CS}_{i r ; s}^{(0)}\right)_{f_{i} f}\left(x_{i}, Y_{i j ; Q}\right)= & \frac{1}{\epsilon^{4}}-\frac{2}{\epsilon^{3}}\left(\ln x_{i}+\Sigma\left(y_{0}, D_{0}^{\prime}-1\right)\right)+\frac{1}{\epsilon^{3}} \gamma_{f_{i}}\left(n_{\mathrm{f}}\right) \\
& +\mathrm{O}\left(\epsilon^{-2}\right) .
\end{aligned}
$$

5. Soft collinear:

$$
\begin{aligned}
\left(\mathrm{CS}_{i r ; s}^{(0)}\right)_{f_{i}}^{(j, l)}\left(x_{i}, Y_{j l ; Q}\right)= & -\frac{1}{\epsilon^{4}}+\frac{2}{\epsilon^{3}}\left(\ln x_{i}+\Sigma\left(y_{0}, D_{0}^{\prime}-1\right)\right)+\frac{1}{\epsilon^{3}}\left(\ln Y_{j l ; Q}-\gamma_{f_{i}}\left(n_{\mathrm{f}}\right)\right) \\
& +\mathrm{O}\left(\epsilon^{-2}\right)
\end{aligned}
$$

for $j, l \neq i$, and

$$
\begin{aligned}
\left(\mathrm{CS}_{i r ; s}^{(0)}\right)_{f_{i}}^{(i, l)}\left(x_{i}, Y_{i l ; Q}\right)= & \frac{5}{6}\left[-\frac{1}{\epsilon^{4}}+\frac{2}{\epsilon^{3}}\left(\ln x_{i}+\Sigma\left(y_{0}, D_{0}^{\prime}-1\right)\right)\right] \\
& +\frac{1}{\epsilon^{3}}\left(\ln Y_{i l ; Q}-\frac{3}{4} \gamma_{f_{i}}\left(n_{\mathrm{f}}\right)\right)+\mathrm{O}\left(\epsilon^{-2}\right)
\end{aligned}
$$

for e.g., $j=i$. 


\begin{tabular}{|c|c|c|c|c|}
\hline colour & $x$ & $1 / \epsilon^{2}$ & $1 / \epsilon^{1}$ & finite \\
\hline \hline \multirow{2}{*}{1} & 1.000 & $-1.870 \pm 0.003$ & $-22.187 \pm 0.020$ & $-76.907 \pm 0.155$ \\
& 0.667 & $2.371 \pm 0.004$ & $-15.435 \pm 0.023$ & $-93.639 \pm 0.156$ \\
\hline \multirow{2}{*}{$\frac{C_{\mathrm{A}}}{C_{\mathrm{F}}}$} & 1.000 & $2.632 \pm 0.002$ & $4.595 \pm 0.013$ & $13.916 \pm 0.095$ \\
& 0.667 & $6.080 \pm 0.002$ & $21.854 \pm 0.014$ & $84.489 \pm 0.100$ \\
\hline$\frac{n_{\mathrm{f}} T_{\mathrm{R}}}{C_{\mathrm{F}}}$ & 1.000 & $-0.611 \pm 0.001$ & $1.159 \pm 0.005$ & $16.629 \pm 0.037$ \\
& 0.667 & $-0.881 \pm 0.001$ & $-1.016 \pm 0.006$ & $7.092 \pm 0.046$ \\
\hline
\end{tabular}

Table 7. Coefficients of the Laurent expansion of $\left(\mathrm{C}_{\text {irs }}^{(0)}\right)_{q}(x)$ for $d_{0}=3-3 \epsilon$ and $\alpha_{0}=1$.

\begin{tabular}{|c|c|c|c|c|}
\hline colour & $x$ & $1 / \epsilon^{2}$ & $1 / \epsilon^{1}$ & finite \\
\hline \hline 1 & 1.000 & $3.664 \pm 0.003$ & $-8.987 \pm 0.029$ & $-42.269 \pm 0.207$ \\
& 0.667 & $11.993 \pm 0.004$ & $19.817 \pm 0.032$ & $30.542 \pm 0.224$ \\
\hline$\frac{n_{\mathrm{f}} T_{\mathrm{R}}}{C_{\mathrm{A}}}$ & 1.000 & $-2.614 \pm 0.005$ & $-5.230 \pm 0.047$ & $-3.361 \pm 0.345$ \\
& 0.667 & $-3.424 \pm 0.006$ & $-11.873 \pm 0.049$ & $-32.821 \pm 0.372$ \\
\hline$\frac{C_{\mathrm{F}} n_{\mathrm{f}} T_{\mathrm{R}}}{C_{\mathrm{A}}^{2}}$ & 1.000 & $-4.798 \pm 0.000$ & $-17.623 \pm 0.003$ & $-45.473 \pm 0.015$ \\
\hline
\end{tabular}

Table 8. Coefficients of the Laurent expansion of $\left(\mathrm{C}_{i r s}^{(0)}\right)_{g}(x)$ for $d_{0}=3-3 \epsilon$ and $\alpha_{0}=1$.

The remaining coefficients in the Laurent expansion are computed numerically. By way of illustration, we present results for the flavour-summed counterterms in tables 7-19 for two kinematic points: one relevant for 2-jet production and the other corresponding to the fully symmetric configuration of final state momenta in 3-jet production. In terms of the invariants introduced in eq. (2.19), these two phase-space points correspond to

$$
\text { 2-jet : } x_{1}=x_{2}=1 \quad \text { and } \quad Y_{12, Q}=1 \text {, }
$$

3-jet symmetric: $\quad x_{1}=x_{2}=x_{3}=\frac{2}{3} \quad$ and $\quad Y_{12, Q}=Y_{13, Q}=Y_{23, Q}=\frac{3}{4}$.

In the numerical computations, we chose the following values for the phase-space cut parameters: $\alpha_{0}=1, y_{0}=1, D_{0}=D_{0}^{\prime}=3$ and $d_{1}=d_{1}^{\prime}=-3$. In tables 7-19, a displayed error estimate of \pm 0.000 implies that the numerical uncertainty for that particular value is smaller than $10^{-3}$.

\subsection{Cross sections to $\mathrm{O}\left(\epsilon^{-2}\right)$}

Using the results presented above together with the results of refs. [25-27, 31, 51], we can check analytically that the $\epsilon$ poles cancel between the doubly virtual contribution and the sum of integrated approximate cross sections in eq. (1.10) to $\mathrm{O}\left(\epsilon^{-2}\right)$ accuracy for the case 


\begin{tabular}{|c|c|c|c|c|c|}
\hline colour & $x$ & $Y$ & $1 / \epsilon^{2}$ & $1 / \epsilon^{1}$ & finite \\
\hline \hline \multirow{2}{*}{1} & 1.000 & 1.000 & $-7.311 \pm 0.003$ & $-3.228 \pm 0.009$ & $-9.300 \pm 0.027$ \\
& 0.667 & 0.750 & $-13.938 \pm 0.004$ & $-20.393 \pm 0.019$ & $-27.573 \pm 0.066$ \\
\hline
\end{tabular}

Table 9. Coefficients of the Laurent expansion of $\left(\mathrm{CS}_{i r ; s}^{(0)}\right)_{q}^{(j, l)}(x, Y)$ for $d_{0}=d_{0}^{\prime}=3-3 \epsilon$ and $\alpha_{0}=y_{0}=1$.

\begin{tabular}{|c|c|c|c|c|c|}
\hline colour & $x$ & $Y$ & $1 / \epsilon^{2}$ & $1 / \epsilon^{1}$ & finite \\
\hline \hline \multirow{2}{*}{1} & 1.000 & 1.000 & $-9.205 \pm 0.003$ & $-8.103 \pm 0.009$ & $-16.717 \pm 0.028$ \\
& 0.667 & 0.750 & $-16.113 \pm 0.004$ & $-27.124 \pm 0.019$ & $-40.577 \pm 0.067$ \\
\hline \multirow{2}{*}{$\frac{n_{\mathrm{f}} T_{\mathrm{R}}}{C_{\mathrm{A}}}$} & 1.000 & 1.000 & $4.454 \pm 0.001$ & $14.204 \pm 0.004$ & $29.039 \pm 0.020$ \\
& 0.667 & 0.750 & $5.017 \pm 0.001$ & $18.479 \pm 0.008$ & $44.490 \pm 0.046$ \\
\hline
\end{tabular}

Table 10. Coefficients of the Laurent expansion of $\left(\mathrm{CS}_{i r ; s}^{(0)}\right)_{g}^{(j, l)}(x, Y)$ for $d_{0}=d_{0}^{\prime}=3-3 \epsilon$ and $\alpha_{0}=y_{0}=1$.

\begin{tabular}{|c|c|c|c|c|c|}
\hline colour & $x$ & $Y$ & $1 / \epsilon^{2}$ & $1 / \epsilon^{1}$ & finite \\
\hline \hline \multirow{2}{*}{1} & 1.000 & 1.000 & $-4.874 \pm 0.002$ & $2.910 \pm 0.007$ & $5.559 \pm 0.024$ \\
& 0.667 & 0.750 & $-10.330 \pm 0.003$ & $-8.326 \pm 0.018$ & $9.292 \pm 0.087$ \\
\hline
\end{tabular}

Table 11. Coefficients of the Laurent expansion of $\left(\mathrm{CS}_{i r ; s}^{(0)}\right)_{q}^{(i r, l)}(x, Y)$ for $d_{0}=d_{0}^{\prime}=3-3 \epsilon$ and $\alpha_{0}=y_{0}=1$.

\begin{tabular}{|c|c|c|c|c|c|}
\hline colour & $x$ & $Y$ & $1 / \epsilon^{2}$ & $1 / \epsilon^{1}$ & finite \\
\hline \hline \multirow{2}{*}{1} & 1.000 & 1.000 & $-6.126 \pm 0.002$ & $0.812 \pm 0.007$ & $7.619 \pm 0.025$ \\
& 0.667 & 0.750 & $-11.839 \pm 0.003$ & $-12.094 \pm 0.018$ & $6.585 \pm 0.089$ \\
\hline \multirow{2}{*}{$\frac{n_{\mathrm{f}} T_{\mathrm{R}}}{C_{\mathrm{A}}}$} & 1.000 & 1.000 & $3.005 \pm 0.000$ & $7.201 \pm 0.002$ & $3.080 \pm 0.017$ \\
& 0.667 & 0.750 & $3.517 \pm 0.001$ & $11.052 \pm 0.007$ & $16.471 \pm 0.045$ \\
\hline
\end{tabular}

Table 12. Coefficients of the Laurent expansion of $\left(\mathrm{CS}_{i r ; s}^{(0)}\right)_{g}^{(i r, l)}(x, Y)$ for $d_{0}=d_{0}^{\prime}=3-3 \epsilon$ and $\alpha_{0}=y_{0}=1$.

of two- and three-jet production in electron-positron annihilation. Here we present the details of these cancellations.

We start by recalling the structure of the integrated approximate cross sections that appear in eq. (1.10). Above we showed that $\int_{2} \mathrm{~d} \sigma_{m+2}^{\mathrm{RR}, \mathrm{A}_{2}}$ can be written as

$$
\int_{2} \mathrm{~d} \sigma_{m+2}^{\mathrm{RR}, \mathrm{A}_{2}}=\mathrm{d} \sigma_{m}^{\mathrm{B}} \otimes \boldsymbol{I}_{2}^{(0)}\left(\{p\}_{m} ; \epsilon\right),
$$

see eq. (3.5). Furthermore, in the present paper and ref. [51], we computed all integrated 


\begin{tabular}{|c|c|c|c|c|}
\hline colour & $x$ & $1 / \epsilon^{2}$ & $1 / \epsilon^{1}$ & finite \\
\hline \hline \multirow{2}{*}{1} & 1.000 & $3.437 \pm 0.001$ & $-6.996 \pm 0.004$ & $-61.629 \pm 0.015$ \\
& 0.667 & $5.886 \pm 0.001$ & $-6.784 \pm 0.005$ & $-100.401 \pm 0.019$ \\
\hline
\end{tabular}

Table 13. Coefficients of the Laurent expansion of $\left(\mathrm{C}_{i r s} \mathrm{CS}_{i r ; s}^{(0)}\right)_{q}(x)$ for $d_{0}=d_{0}^{\prime}=3-3 \epsilon$ and $\alpha_{0}=y_{0}=1$.

\begin{tabular}{|c|c|c|c|c|}
\hline colour & $x$ & $1 / \epsilon^{2}$ & $1 / \epsilon^{1}$ & finite \\
\hline \hline \multirow{2}{*}{1} & 1.000 & $4.048 \pm 0.001$ & $-6.973 \pm 0.004$ & $-66.612 \pm 0.016$ \\
& 0.667 & $6.633 \pm 0.001$ & $-5.975 \pm 0.005$ & $-103.489 \pm 0.019$ \\
\hline \multirow{2}{*}{$\frac{n_{\mathrm{f}} T_{\mathrm{R}}}{C_{\mathrm{A}}}$} & 1.000 & $-1.556 \pm 0.000$ & $-1.601 \pm 0.001$ & $8.364 \pm 0.011$ \\
& 0.667 & $-1.826 \pm 0.000$ & $-3.445 \pm 0.001$ & $2.731 \pm 0.011$ \\
\hline
\end{tabular}

Table 14. Coefficients of the Laurent expansion of $\left(\mathrm{C}_{i r s} \mathrm{CS}_{i r ; s}^{(0)}\right)_{g}(x)$ for $d_{0}=d_{0}^{\prime}=3-3 \epsilon$ and $\alpha_{0}=y_{0}=1$.

\begin{tabular}{|c|c|c|c|c|c|}
\hline colour & $x$ & $Y$ & $1 / \epsilon^{2}$ & $1 / \epsilon^{1}$ & finite \\
\hline \hline \multirow{2}{*}{1} & 1.000 & 1.000 & $8.311 \pm 0.003$ & $11.149 \pm 0.010$ & $40.832 \pm 0.028$ \\
& 0.667 & 0.750 & $13.099 \pm 0.002$ & $24.109 \pm 0.011$ & $61.583 \pm 0.031$ \\
\hline
\end{tabular}

Table 15. Coefficients of the Laurent expansion of $\left(\mathrm{C}_{i r ; j s} \mathrm{CS}_{i r ; s}^{(0)}\right)_{q f}(x, Y)$ for $d_{0}=d_{0}^{\prime}=3-3 \epsilon$ and $\alpha_{0}=y_{0}=1$.

\begin{tabular}{|c|c|c|c|c|c|}
\hline colour & $x$ & $Y$ & $1 / \epsilon^{2}$ & $1 / \epsilon^{1}$ & finite \\
\hline \hline \multirow{2}{*}{1} & 1.000 & 1.000 & $10.205 \pm 0.003$ & $16.358 \pm 0.010$ & $51.240 \pm 0.029$ \\
& 0.667 & 0.750 & $15.179 \pm 0.002$ & $30.471 \pm 0.011$ & $75.387 \pm 0.032$ \\
\hline \multirow{2}{*}{$\frac{n_{\mathrm{f}} T_{\mathrm{R}}}{C_{\mathrm{A}}}$} & 1.000 & 1.000 & $-4.454 \pm 0.001$ & $-14.871 \pm 0.004$ & $-35.687 \pm 0.020$ \\
& 0.667 & 0.750 & $-4.825 \pm 0.001$ & $-17.549 \pm 0.004$ & $-45.158 \pm 0.022$ \\
\hline
\end{tabular}

Table 16. Coefficients of the Laurent expansion of $\left(\mathrm{C}_{i r ; j s} \mathrm{CS}_{i r ; s}^{(0)}\right)_{g f}(x, Y)$ for $d_{0}=d_{0}^{\prime}=3-3 \epsilon$ and $\alpha_{0}=y_{0}=1$.

counterterms which enter the insertion operator $\boldsymbol{I}_{2}^{(0)}\left(\{p\}_{m} ; \epsilon\right)$. Next, $\int_{2} \mathrm{~d} \sigma_{m+2}^{\mathrm{RR}, \mathrm{A}_{12}}$ was computed in ref. [31], and we found

$$
\int_{2} \mathrm{~d} \sigma_{m+2}^{\mathrm{RR}, \mathrm{A}_{12}}=\mathrm{d} \sigma_{m}^{\mathrm{B}} \otimes \boldsymbol{I}_{12}^{(0)}\left(\{p\}_{m} ; \epsilon\right)
$$

where we also computed all integrated counterterms that are used to build up the insertion operator $\boldsymbol{I}_{12}^{(0)}\left(\{p\}_{m} ; \epsilon\right)$. Finally, the form of $\int_{1} \mathrm{~d} \sigma_{m+1}^{\mathrm{RV}, \mathrm{A}_{1}}$ and $\int_{1}\left(\int_{1} \mathrm{~d} \sigma_{m+2}^{\mathrm{RR}, \mathrm{A}_{1}}\right)^{\mathrm{A}_{1}}$ was derived 


\begin{tabular}{|c|c|c|c|c|c|}
\hline colour & $x_{1}$ & $x_{2}$ & $1 / \epsilon^{2}$ & $1 / \epsilon^{1}$ & finite \\
\hline \hline \multirow{2}{*}{1} & 1.000 & 1.000 & $0.162 \pm 0.000$ & $-4.108 \pm 0.003$ & $5.189 \pm 0.013$ \\
& 0.667 & 0.667 & $4.408 \pm 0.001$ & $5.732 \pm 0.004$ & $18.494 \pm 0.018$ \\
\hline
\end{tabular}

Table 17. Coefficients of the Laurent expansion of $\left(\mathrm{C}_{i r ; j s}^{(0)}\right)_{q q}\left(x_{1}, x_{2}\right)$ for $d_{0}=3-3 \epsilon$ and $\alpha_{0}=1$.

\begin{tabular}{|c|c|c|c|c|c|}
\hline colour & $x_{1}$ & $x_{2}$ & $1 / \epsilon^{2}$ & $1 / \epsilon^{1}$ & finite \\
\hline \hline \multirow{2}{*}{1} & 1.000 & 1.000 & $0.859 \pm 0.000$ & $-3.131 \pm 0.003$ & $5.070 \pm 0.013$ \\
& 0.667 & 0.667 & $5.333 \pm 0.001$ & $8.076 \pm 0.004$ & $22.304 \pm 0.019$ \\
\hline \multirow{2}{*}{$\frac{n_{\mathrm{f}} T_{\mathrm{R}}}{C_{\mathrm{A}}}$} & 1.000 & 1.000 & $-1.727 \pm 0.000$ & $-3.683 \pm 0.002$ & $-3.445 \pm 0.008$ \\
& 0.667 & 0.667 & $-2.183 \pm 0.000$ & $-6.871 \pm 0.002$ & $-14.493 \pm 0.011$ \\
\hline
\end{tabular}

Table 18. Coefficients of the Laurent expansion of $\left(\mathrm{C}_{i r ; j s}^{(0)}\right)_{q g}\left(x_{1}, x_{2}\right)$ for $d_{0}=3-3 \epsilon$ and $\alpha_{0}=1$, with $\left(\mathrm{C}_{i r ; j s}^{(0)}\right)_{g q}\left(x_{1}, x_{2}\right)=\left(\mathrm{C}_{i r ; j s}^{(0)}\right)_{q g}\left(x_{2}, x_{1}\right)$.

\begin{tabular}{|c|c|c|c|c|c|}
\hline colour & $x_{1}$ & $x_{2}$ & $1 / \epsilon^{2}$ & $1 / \epsilon^{1}$ & finite \\
\hline \hline \multirow{2}{*}{1} & 1.000 & 1.000 & $1.612 \pm 0.000$ & $-1.855 \pm 0.003$ & $5.670 \pm 0.013$ \\
& 0.667 & 0.667 & $6.314 \pm 0.001$ & $10.780 \pm 0.004$ & $27.243 \pm 0.019$ \\
\hline \multirow{2}{*}{$\frac{n_{\mathrm{f}} T_{\mathrm{R}}}{C_{\mathrm{A}}}$} & 1.000 & 1.000 & $-3.677 \pm 0.000$ & $-8.780 \pm 0.003$ & $-11.184 \pm 0.011$ \\
& 0.667 & 0.667 & $-4.589 \pm 0.000$ & $-15.404 \pm 0.002$ & $-35.158 \pm 0.016$ \\
\hline$\frac{n_{\mathrm{f}}^{2} T_{\mathrm{R}}^{2}}{C_{\mathrm{A}}^{2}}$ & 1.000 & 1.000 & $0.222 \pm 0.000$ & $1.637 \pm 0.000$ & $5.932 \pm 0.003$ \\
& 0.667 & 0.667 & $0.222 \pm 0.000$ & $1.884 \pm 0.000$ & $8.057 \pm 0.004$ \\
\hline
\end{tabular}

Table 19. Coefficients of the Laurent expansion of $\left(\mathrm{C}_{i r ; j s}^{(0)}\right)_{g g}\left(x_{1}, x_{2}\right)$ for $d_{0}=3-3 \epsilon$ and $\alpha_{0}=1$.

in ref. [25]. We have

$$
\int_{1} \mathrm{~d} \sigma_{m+1}^{\mathrm{RV}, \mathrm{A}_{1}}=\mathrm{d} \sigma_{m}^{\mathrm{V}} \otimes \boldsymbol{I}_{1}^{(0)}\left(\{p\}_{m} ; \epsilon\right)+\mathrm{d} \sigma_{m}^{\mathrm{B}} \otimes \boldsymbol{I}_{1}^{(1)}\left(\{p\}_{m} ; \epsilon\right),
$$

and

$$
\int_{1}\left(\int_{1} \mathrm{~d} \sigma_{m+2}^{\mathrm{RR}, \mathrm{A}_{1}}\right)^{\mathrm{A}_{1}}=\mathrm{d} \sigma_{m}^{\mathrm{B}} \otimes\left[\frac{1}{2}\left\{\boldsymbol{I}_{1}^{(0)}\left(\{p\}_{m} ; \epsilon\right), \boldsymbol{I}_{1}^{(0)}\left(\{p\}_{m} ; \epsilon\right)\right\}+\boldsymbol{I}_{1}^{R \times(0)}\left(\{p\}_{m} ; \epsilon\right)\right],
$$

where the integrated counterterms entering the insertion operators $\boldsymbol{I}_{1}^{(l)}\left(\{p\}_{m} ; \epsilon\right), l=0,1$ and $\boldsymbol{I}_{1}^{R \times(0)}\left(\{p\}_{m} ; \epsilon\right)$ were computed in refs. [25-27].

Turning to specific processes, we consider first $e^{+} e^{-} \rightarrow 2$ jets. The only Born level partonic subprocess to consider is the production of a quark-antiquark pair and we denote the corresponding Born cross section as $\mathrm{d} \sigma_{2}^{\mathrm{B}}\left(p_{1}, p_{2}\right)$. The flavours are assigned such that 
the quark carries label 1 and the antiquark label 2. The kinematics and colour algebra are both trivial. On the one hand, momentum conservation implies

$$
x_{1}=x_{2}=Y_{12, Q}=y_{12}=1 .
$$

On the other hand, colour conservation gives

$$
\boldsymbol{T}_{1} \boldsymbol{T}_{2}=-C_{\mathrm{F}},
$$

while $C_{f_{1}}=C_{f_{2}}=C_{\mathrm{F}}$. Because of this, the pole parts of the virtual and double virtual cross sections are simply proportional to the Born cross section [55], ${ }^{4}$

$$
\mathrm{d} \sigma_{2}^{\mathrm{V}}\left(p_{1}, p_{2}\right)=\frac{\alpha_{\mathrm{s}}}{2 \pi} S_{\epsilon}\left(\frac{\mu^{2}}{Q^{2}}\right)^{\epsilon}\left\{-\frac{2}{\epsilon^{2}}-\frac{3}{\epsilon}+\mathrm{O}\left(\epsilon^{0}\right)\right\} C_{\mathrm{F}} \mathrm{d} \sigma_{2}^{\mathrm{B}}\left(p_{1}, p_{2}\right)
$$

(used in eq. (5.12)) and

$$
\begin{aligned}
\mathrm{d} \sigma_{2}^{\mathrm{VV}}\left(p_{1}, p_{2}\right)=\left[\frac{\alpha_{\mathrm{s}}}{2 \pi} S_{\epsilon}\left(\frac{\mu^{2}}{Q^{2}}\right)^{\epsilon}\right]^{2}\{ & \frac{2}{\epsilon^{4}}+\left(6+\frac{11}{4} \frac{C_{\mathrm{A}}}{C_{\mathrm{F}}}-\frac{T_{\mathrm{R}} n_{\mathrm{f}}}{C_{\mathrm{F}}}\right) \frac{1}{\epsilon^{3}} \\
& \left.+\mathrm{O}\left(\epsilon^{-2}\right)\right\} C_{\mathrm{F}}^{2} \mathrm{~d} \sigma_{2}^{\mathrm{B}}\left(p_{1}, p_{2}\right) .
\end{aligned}
$$

We now present the explicit expressions for the integrated approximate cross sections, starting with $\int_{2} \mathrm{~d} \sigma_{4}^{\mathrm{RR}, \mathrm{A}_{2}}$. The insertion operator $\boldsymbol{I}_{2}^{(0)}\left(p_{1}, p_{2}\right)$ relevant for two-jet production was evaluated analytically to $\mathrm{O}\left(\epsilon^{-2}\right)$ accuracy in ref. [51], and we find

$$
\begin{aligned}
\int_{2} \mathrm{~d} \sigma_{4}^{\mathrm{RR}, \mathrm{A}_{2}}=\left[\frac{\alpha_{\mathrm{s}}}{2 \pi} S_{\epsilon}\left(\frac{\mu^{2}}{Q^{2}}\right)^{\epsilon}\right]^{2}\{ & \left(2+\frac{C_{\mathrm{A}}}{2 C_{\mathrm{F}}}\right) \frac{1}{\epsilon^{4}}+\left(6+\frac{29 C_{\mathrm{A}}}{12 C_{\mathrm{F}}}-\frac{T_{\mathrm{R}} n_{\mathrm{f}}}{3 C_{\mathrm{F}}}\right) \frac{1}{\epsilon^{3}} \\
& \left.+\mathrm{O}\left(\epsilon^{-2}\right)\right\} C_{\mathrm{F}}^{2} \mathrm{~d} \sigma_{2}^{\mathrm{B}}\left(p_{1}, p_{2}\right) .
\end{aligned}
$$

Next, the insertion operator $\boldsymbol{I}_{12}^{(0)}\left(p_{1}, p_{2}\right)$ relevant for constructing $\int_{2} \mathrm{~d} \sigma_{4}^{\mathrm{RR}, \mathrm{A}_{12}}$ was computed in ref. [31] and we have ${ }^{5}$

$$
\begin{aligned}
& \int_{2} \mathrm{~d} \sigma_{4}^{\mathrm{RR}, \mathrm{A}_{12}}=\left[\frac{\alpha_{\mathrm{s}}}{2 \pi} S_{\epsilon}\left(\frac{\mu^{2}}{Q^{2}}\right)^{\epsilon}\right]^{2}\left\{\frac{4}{\epsilon^{4}}+\left(12+\frac{10 C_{\mathrm{A}}}{3 C_{\mathrm{F}}}-\frac{2 T_{\mathrm{R}} n_{\mathrm{f}}}{3 C_{\mathrm{F}}}+\frac{2 C_{\mathrm{A}}}{C_{\mathrm{F}}} \Sigma\left(y_{0}, 3\right)\right) \frac{1}{\epsilon^{3}}\right. \\
& \left.+\mathrm{O}\left(\epsilon^{-2}\right)\right\} C_{\mathrm{F}}^{2} \mathrm{~d} \sigma_{2}^{\mathrm{B}}\left(p_{1}, p_{2}\right) .
\end{aligned}
$$

Turning to $\int_{1} \mathrm{~d} \sigma_{3}^{\mathrm{RV}, \mathrm{A}_{1}}$ and $\int_{1}\left(\int_{1} \mathrm{~d} \sigma_{4}^{\mathrm{RR}, \mathrm{A}_{1}}\right)^{\mathrm{A}_{1}}$, using the results for the integrated counterterms in refs. [25-27], we find

$$
\begin{aligned}
\int_{1} \mathrm{~d} \sigma_{3}^{\mathrm{RV}, \mathrm{A}_{1}}=\left[\frac{\alpha_{\mathrm{s}}}{2 \pi} S_{\epsilon}\left(\frac{\mu^{2}}{Q^{2}}\right)^{\epsilon}\right]^{2}\{ & -\left(4+\frac{C_{\mathrm{A}}}{2 C_{\mathrm{F}}}\right) \frac{1}{\epsilon^{4}}-\left(12+\frac{31 C_{\mathrm{A}}}{6 C_{\mathrm{F}}}-\frac{4 T_{\mathrm{R}} n_{\mathrm{f}}}{3 C_{\mathrm{F}}}\right) \frac{1}{\epsilon^{3}} \\
& \left.+\mathrm{O}\left(\epsilon^{-2}\right)\right\} C_{\mathrm{F}}^{2} \mathrm{~d} \sigma_{2}^{\mathrm{B}}\left(p_{1}, p_{2}\right)
\end{aligned}
$$

\footnotetext{
${ }^{4}$ We include a factor of $S_{\epsilon}$ in the definition of the running coupling $\alpha_{\mathrm{s}}$ as in ref. [2]. In ref. [55] the running coupling is defined without this factor, hence the different prefactors.

${ }^{5}$ Note that there is an error in eq. (3.36) of ref. [31] in the colour factor of the second term on the right hand side, hence the correct expression for the insertion operator is the one reported in eq. (5.19).
} 
and

$$
\begin{gathered}
\int_{1}\left(\int_{1} \mathrm{~d} \sigma_{4}^{\mathrm{RR}, \mathrm{A}_{1}}\right)^{\mathrm{A}_{1}}=\left[\frac{\alpha_{\mathrm{s}}}{2 \pi} S_{\epsilon}\left(\frac{\mu^{2}}{Q^{2}}\right)^{\epsilon}\right]^{2}\left\{\frac{4}{\epsilon^{4}}+\left(12+\frac{10 C_{\mathrm{A}}}{3 C_{\mathrm{F}}}-\frac{2 T_{\mathrm{R}} n_{\mathrm{f}}}{3 C_{\mathrm{F}}}+\frac{2 C_{\mathrm{A}}}{C_{\mathrm{F}}} \Sigma\left(y_{0}, 3\right)\right) \frac{1}{\epsilon^{3}}\right. \\
\left.+\mathrm{O}\left(\epsilon^{-2}\right)\right\} C_{\mathrm{F}}^{2} \mathrm{~d} \sigma_{2}^{\mathrm{B}}\left(p_{1}, p_{2}\right)
\end{gathered}
$$

respectively. Using eqs. (5.17)-(5.21), it is straightforward to check that indeed the $\epsilon$ poles cancel to $\mathrm{O}\left(\epsilon^{-2}\right)$ accuracy, i.e.,

$$
\mathrm{d} \sigma_{2}^{\mathrm{VV}}+\int_{2} \mathrm{~d} \sigma_{4}^{\mathrm{RR}, \mathrm{A}_{2}}-\int_{2} \mathrm{~d} \sigma_{4}^{\mathrm{RR}, \mathrm{A}_{12}}+\int_{1} \mathrm{~d} \sigma_{3}^{\mathrm{RV}, \mathrm{A}_{1}}+\int_{1}\left(\int_{1} \mathrm{~d} \sigma_{4}^{\mathrm{RR}, \mathrm{A}_{1}}\right)^{\mathrm{A}_{1}}=\mathrm{O}\left(\epsilon^{-2}\right) .
$$

Next, we consider the process $e^{+} e^{-} \rightarrow 3$ jets. There is again only a single Born level partonic subprocess to consider, the production of a quark-antiquark pair and a gluon. We write the corresponding Born cross section as $\mathrm{d} \sigma_{3}^{\mathrm{B}}\left(p_{1}, p_{2}, p_{3}\right)$, and assign the flavours as follows: the quark carries label 1 the antiquark label 2 and the gluon label 3. The colour algebra is again trivial and colour conservation implies

$$
\boldsymbol{T}_{1} \boldsymbol{T}_{2}=\frac{C_{\mathrm{A}}-2 C_{\mathrm{F}}}{2}, \quad \boldsymbol{T}_{1} \boldsymbol{T}_{3}=\boldsymbol{T}_{2} \boldsymbol{T}_{3}=-\frac{C_{\mathrm{A}}}{2},
$$

while $C_{f_{1}}=C_{f_{2}}=C_{\mathrm{F}}$ and $C_{f_{3}}=C_{\mathrm{A}}$. Thus, the pole parts of the virtual and double virtual cross sections are just proportional to the Born cross section [55]

$$
\begin{aligned}
\mathrm{d} \sigma_{3}^{\mathrm{V}}\left(p_{1}, p_{2}, p_{3}\right)= & \frac{\alpha_{\mathrm{s}}}{2 \pi} S_{\epsilon}\left(\frac{\mu^{2}}{Q^{2}}\right)^{\epsilon}\left\{-\left(2+\frac{C_{\mathrm{A}}}{C_{\mathrm{F}}}\right) \frac{1}{\epsilon^{2}}-\left[3+\frac{11 C_{\mathrm{A}}}{6 C_{\mathrm{F}}}-\frac{2 T_{\mathrm{R}} n_{\mathrm{f}}}{3 C_{\mathrm{F}}}\right.\right. \\
& \left.-\left(2-\frac{C_{\mathrm{A}}}{C_{\mathrm{F}}}\right) \ln y_{12}-\frac{C_{\mathrm{A}}}{C_{\mathrm{F}}}\left(\ln y_{13}+\ln y_{23}\right)\right] \frac{1}{\epsilon} \\
& \left.+\mathrm{O}\left(\epsilon^{0}\right)\right\} C_{\mathrm{F}} \mathrm{d} \sigma_{3}^{\mathrm{B}}\left(p_{1}, p_{2}, p_{3}\right)
\end{aligned}
$$

(used in eq. (5.12)) where we use the same normalisation as in eq. (5.16) and

$$
\begin{aligned}
\mathrm{d} \sigma_{3}^{\mathrm{VV}}\left(p_{1}, p_{2}, p_{3}\right)= & {\left[\frac{\alpha_{\mathrm{s}}}{2 \pi} S_{\epsilon}\left(\frac{\mu^{2}}{Q^{2}}\right)^{\epsilon}\right]^{2}\left\{\left(2+\frac{C_{\mathrm{A}}}{C_{\mathrm{F}}}\right)^{2} \frac{1}{2 \epsilon^{4}}+\left(2+\frac{C_{\mathrm{A}}}{C_{\mathrm{F}}}\right)\left[3+\frac{77 C_{\mathrm{A}}}{24 C_{\mathrm{F}}}\right.\right.} \\
& \left.-\frac{7 T_{\mathrm{R}} n_{\mathrm{f}}}{6 C_{\mathrm{F}}}-\left(2-\frac{C_{\mathrm{A}}}{C_{\mathrm{F}}}\right) \ln y_{12}-\frac{C_{\mathrm{A}}}{C_{\mathrm{F}}}\left(\ln y_{13}+\ln y_{23}\right)\right] \frac{1}{\epsilon} \\
& \left.+\mathrm{O}\left(\epsilon^{-2}\right)\right\} C_{\mathrm{F}}^{2} \mathrm{~d} \sigma_{3}^{\mathrm{B}}\left(p_{1}, p_{2}, p_{3}\right) .
\end{aligned}
$$

We now present the integrated approximate cross sections, starting with $\int_{2} \mathrm{~d} \sigma_{5}^{\mathrm{RR}, \mathrm{A}_{2}}$. With the results of the present paper and ref. [51], we obtain

$$
\begin{aligned}
\int_{2} \mathrm{~d} \sigma_{5}^{\mathrm{RR}, \mathrm{A}_{2}}= & {\left[\frac{\alpha_{\mathrm{s}}}{2 \pi} S_{\epsilon}\left(\frac{\mu^{2}}{Q^{2}}\right)^{\epsilon}\right]^{2}\left\{\left(2+\frac{5 C_{\mathrm{A}}}{2 C_{\mathrm{F}}}+\frac{3 C_{\mathrm{A}}^{2}}{4 C_{\mathrm{F}}^{2}}\right) \frac{1}{\epsilon^{4}}+\left[6+\frac{109 C_{\mathrm{A}}}{12 C_{\mathrm{F}}}+\frac{77 C_{\mathrm{A}}^{2}}{24 C_{\mathrm{F}}^{2}}\right.\right.} \\
& -\frac{7 T_{\mathrm{R}} n_{\mathrm{f}}}{3 C_{\mathrm{F}}}-\frac{C_{\mathrm{A}} T_{\mathrm{R}} n_{\mathrm{f}}}{2 C_{\mathrm{F}}^{2}}-\left(4+\frac{C_{\mathrm{A}}}{C_{\mathrm{F}}}-\frac{3 C_{\mathrm{A}}^{2}}{2 C_{\mathrm{F}}^{2}}\right) \ln y_{12} \\
& \left.\left.-\left(\frac{2 C_{\mathrm{A}}}{C_{\mathrm{F}}}+\frac{3 C_{\mathrm{A}}^{2}}{2 C_{\mathrm{F}}^{2}}\right)\left(\ln y_{13}+\ln y_{23}\right)\right] \frac{1}{\epsilon^{3}}+\mathrm{O}\left(\epsilon^{-2}\right)\right\} C_{\mathrm{F}}^{2} \mathrm{~d} \sigma_{3}^{\mathrm{B}}\left(p_{1}, p_{2}, p_{3}\right) .
\end{aligned}
$$


For $\int_{2} \mathrm{~d} \sigma_{5}^{\mathrm{RR}, \mathrm{A}_{12}}$ we find using ref. [31] $]^{6}$

$$
\begin{aligned}
\int_{2} \mathrm{~d} \sigma_{5}^{\mathrm{RR}, \mathrm{A}_{12}=} & {\left[\frac{\alpha_{\mathrm{s}}}{2 \pi} S_{\epsilon}\left(\frac{\mu^{2}}{Q^{2}}\right)^{\epsilon}\right]^{2}\left\{\left(2+\frac{C_{\mathrm{A}}}{C_{\mathrm{F}}}\right)^{2} \frac{1}{\epsilon^{4}}+\left[12+\frac{50 C_{\mathrm{A}}}{3 C_{\mathrm{F}}}+\frac{11 C_{\mathrm{A}}^{2}}{2 C_{\mathrm{F}}^{2}}\right.\right.} \\
& -\frac{4 T_{\mathrm{R}} n_{\mathrm{f}}}{C_{\mathrm{F}}}-\frac{4 C_{\mathrm{A}} T_{\mathrm{R}} n_{\mathrm{f}}}{3 C_{\mathrm{F}}^{2}}-\left(8+\frac{C_{\mathrm{A}}}{C_{\mathrm{F}}}-\frac{5 C_{\mathrm{A}}^{2}}{2 C_{\mathrm{F}}^{2}}\right) \ln y_{12} \\
& \left.-\left(\frac{4 C_{\mathrm{A}}}{C_{\mathrm{F}}}+\frac{5 C_{\mathrm{A}}^{2}}{2 C_{\mathrm{F}}^{2}}\right)\left(\ln y_{13}+\ln y_{23}\right)+\left(\frac{2 C_{\mathrm{A}}}{C_{\mathrm{F}}}+\frac{C_{\mathrm{A}}^{2}}{C_{\mathrm{F}}^{2}}\right) \Sigma\left(y_{0}, 3\right)\right] \frac{1}{\epsilon^{3}} \\
& \left.+\mathrm{O}\left(\epsilon^{-2}\right)\right\} C_{\mathrm{F}}^{2} \mathrm{~d} \sigma_{3}^{\mathrm{B}}\left(p_{1}, p_{2}, p_{3}\right) .
\end{aligned}
$$

Finally, turning to $\int_{1} \mathrm{~d} \sigma_{4}^{\mathrm{RV}, \mathrm{A}_{1}}$ and $\int_{1}\left(\int_{1} \mathrm{~d} \sigma_{5}^{\mathrm{RR}, \mathrm{A}_{1}}\right)^{\mathrm{A}_{1}}$, we have

$$
\begin{aligned}
\int_{1} \mathrm{~d} \sigma_{4}^{\mathrm{RV}, \mathrm{A}_{1}}= & {\left[\frac{\alpha_{\mathrm{s}}}{2 \pi} S_{\epsilon}\left(\frac{\mu^{2}}{Q^{2}}\right)^{\epsilon}\right]^{2}\left\{-\left(4+\frac{9 C_{\mathrm{A}}}{2 C_{\mathrm{F}}}+\frac{5 C_{\mathrm{A}}^{2}}{4 C_{\mathrm{F}}^{2}}\right) \frac{1}{\epsilon^{4}}-\left[12+\frac{37 C_{\mathrm{A}}}{2 C_{\mathrm{F}}}+\frac{77 C_{\mathrm{A}}^{2}}{12 C_{\mathrm{F}}^{2}}\right.\right.} \\
& -\frac{14 T_{\mathrm{R}} n_{\mathrm{f}}}{3 C_{\mathrm{F}}}-\frac{5 C_{\mathrm{A}} T_{\mathrm{R}} n_{\mathrm{f}}}{3 C_{\mathrm{F}}^{2}}-\left(8+\frac{C_{\mathrm{A}}}{C_{\mathrm{F}}}-\frac{5 C_{\mathrm{A}}^{2}}{2 C_{\mathrm{F}}^{2}}\right) \ln y_{12} \\
& \left.\left.-\left(\frac{4 C_{\mathrm{A}}}{C_{\mathrm{F}}}+\frac{5 C_{\mathrm{A}}^{2}}{2 C_{\mathrm{F}}^{2}}\right)\left(\ln y_{13}+\ln y_{23}\right)\right] \frac{1}{\epsilon^{3}}+\mathrm{O}\left(\epsilon^{-2}\right)\right\} C_{\mathrm{F}}^{2} \mathrm{~d} \sigma_{3}^{\mathrm{B}}\left(p_{1}, p_{2}, p_{3}\right)
\end{aligned}
$$

and

$$
\begin{aligned}
\int_{1}\left(\int_{1} \mathrm{~d} \sigma_{5}^{\mathrm{RR}, \mathrm{A}_{1}}\right)^{\mathrm{A}_{1}}= & {\left[\frac{\alpha_{\mathrm{s}}}{2 \pi} S_{\epsilon}\left(\frac{\mu^{2}}{Q^{2}}\right)^{\epsilon}\right]^{2}\left\{\left(2+\frac{C_{\mathrm{A}}}{C_{\mathrm{F}}}\right)^{2} \frac{1}{\epsilon^{4}}+\left[12+\frac{50 C_{\mathrm{A}}}{3 C_{\mathrm{F}}}+\frac{11 C_{\mathrm{A}}^{2}}{2 C_{\mathrm{F}}^{2}}\right.\right.} \\
& -\frac{4 T_{\mathrm{R}} n_{\mathrm{f}}}{C_{\mathrm{F}}}-\frac{4 C_{\mathrm{A}} T_{\mathrm{R}} n_{\mathrm{f}}}{3 C_{\mathrm{F}}^{2}}-\left(8+\frac{C_{\mathrm{A}}}{C_{\mathrm{F}}}-\frac{5 C_{\mathrm{A}}^{2}}{2 C_{\mathrm{F}}^{2}}\right) \ln y_{12} \\
& \left.-\left(\frac{4 C_{\mathrm{A}}}{C_{\mathrm{F}}}+\frac{5 C_{\mathrm{A}}^{2}}{2 C_{\mathrm{F}}^{2}}\right)\left(\ln y_{13}+\ln y_{23}\right)+\left(\frac{2 C_{\mathrm{A}}}{C_{\mathrm{F}}}+\frac{C_{\mathrm{A}}^{2}}{C_{\mathrm{F}}^{2}}\right) \Sigma\left(y_{0}, 3\right)\right] \frac{1}{\epsilon^{3}} \\
& \left.+\mathrm{O}\left(\epsilon^{-2}\right)\right\} C_{\mathrm{F}}^{2} \mathrm{~d} \sigma_{3}^{\mathrm{B}}\left(p_{1}, p_{2}, p_{3}\right)
\end{aligned}
$$

where the result of refs. [25-27] have been used. We note that although the integrated counterterms themselves are functions of the kinematic variables $x_{i}$ and $Y_{i k, Q}$ (see eq. (2.19)) as in e.g., eqs. (5.2)-(5.7), the integrated approximate cross sections in eqs. (5.26)-(5.29) nevertheless depend on the kinematics through the usual two-particle invariants, $y_{i k}$. It is again elementary to check with eqs. (5.25)-(5.29) that the $\epsilon$ poles indeed cancel to $\mathrm{O}\left(\epsilon^{-2}\right)$ accuracy, i.e.,

$$
\mathrm{d} \sigma_{3}^{\mathrm{VV}}+\int_{2} \mathrm{~d} \sigma_{5}^{\mathrm{RR}, \mathrm{A}_{2}}-\int_{2} \mathrm{~d} \sigma_{5}^{\mathrm{RR}, \mathrm{A}_{12}}+\int_{1} \mathrm{~d} \sigma_{4}^{\mathrm{RV}, \mathrm{A}_{1}}+\int_{1}\left(\int_{1} \mathrm{~d} \sigma_{5}^{\mathrm{RR}, \mathrm{A}_{1}}\right)^{\mathrm{A}_{1}}=\mathrm{O}\left(\epsilon^{-2}\right) .
$$

To finish, let us comment that although we have only shown the cancellation of $\epsilon$ poles here up to $\mathrm{O}\left(\epsilon^{-2}\right)$, this is already a very strong check on the correctness of the results, considering that we computed and combined hundreds of master integrals to arrive at the final formulae presented above.

\footnotetext{
${ }^{6}$ See footnote 5 regarding an error in ref. [31]. The correct result is reported in eq. (5.27) here.
} 


\section{Conclusions}

We have computed the integrals over the two-particle factorised phase space of the collineartype contributions to the doubly unresolved counterterm of the NNLO subtraction formalism, defined in refs. [22, 23]. We presented those integrals in terms of parametric representations that are suitable for evaluation with sector decomposition. After evaluating them, we checked the numerical results with the publicly available code SecDec [56], always finding agreement within the numerical uncertainty of the integrations. The soft-type contributions are presented in a companion paper [51].

By these two papers, we complete the integration of the subtraction terms over the unresolved phase spaces, and the computation of the finite cross section $\mathrm{d} \sigma_{m}^{\mathrm{NNLO}}$ in eq. (1.10) becomes feasible for electron-positron annihilation into two and three jets. We have explicitly demonstrated the cancellation of $\epsilon$ poles in $\mathrm{d} \sigma_{m}^{\mathrm{NNLO}}$ for $m=2$ and 3 up to $\mathrm{O}\left(\epsilon^{-2}\right)$. Although the formalism is complete, for a higher number of jets some more work is required, because some integrals were evaluated specifically for three-jet kinematics.

\section{Acknowledgments}

This research was supported by the LHCPhenoNet network PITN-GA-2010-264564, the TÁMOP 4.2.1./B-09/1/KONV-2010-0007 project, the Hungarian Scientific Research Fund grant K-101482 and the Swiss National Science Foundation Joint Research Project SCOPES IZ73Z0_1/28079. We are grateful to Francesco Tramontano for useful discussions. G.S. is grateful to the INFN Laboratori Nazionali di Frascati for the hospitality during the late stages of the work.

\section{A Modified doubly real subtraction terms}

We outline a simple modification to the NNLO subtraction scheme presented in refs. [22, 23]. Parts of these modifications were introduced previously: those relevant to the singly unresolved approximate cross section $\mathrm{d} \sigma_{m+2}^{\mathrm{RR}, \mathrm{A}_{1}}$ in eq. (1.8), and to the approximate cross sections in eq. (1.9), were introduced in ref. [25], while those relevant to the iterated doubly unresolved approximate cross section $\mathrm{d} \sigma_{m+2}^{\mathrm{RR}, \mathrm{A}_{12}}$ appearing in eq. (1.8) were presented in ref. [31].

Recall that the doubly unresolved approximate cross section can be written symbolically as in eq. (3.1)

$$
\mathrm{d} \sigma_{m+2}^{\mathrm{RR}, \mathrm{A}_{2}}=\mathrm{d} \phi_{m}\left[\mathrm{~d} p_{2}\right] \mathcal{A}_{2}^{(0)}\left|\mathcal{M}_{m+2}^{(0)}\right|^{2},
$$

where the doubly unresolved approximation is a sum of terms (see eq. (3.2)). The precise definition of these terms involves the momentum mappings discussed in section 2.3. All such mappings lead to an exact factorisation of the $m+2$-particle phase space, symbolically written as

$$
\mathrm{d} \phi_{m+2}(\{p\} ; Q)=\mathrm{d} \phi_{m}\left(\{\tilde{p}\}_{m} ; Q\right)\left[\mathrm{d} p_{2 ; m}\right] .
$$


The only feature of the factorised phase spaces $\left[\mathrm{d} p_{2 ; m}\right]$ that is relevant presently is that they carry a dependence on the number of partons, $m$, of the form

$$
\begin{aligned}
{\left[\mathrm{d} p_{2 ; m}^{(i r s)}\right] } & \propto\left(1-\alpha_{i r s}\right)^{2(m-1)(1-\epsilon)-1}, \\
{\left[\mathrm{~d} p_{2 ; m}^{(i r ; j s)}\right] } & \propto\left(1-\alpha_{i r}-\alpha_{j s}\right)^{2(m-1)(1-\epsilon)}, \\
{\left[\mathrm{d} p_{2 ; m}^{(r s)}\right] } & \propto\left(1-y_{r Q}-y_{s Q}+y_{r s}\right)^{(m-1)(1-\epsilon)-1},
\end{aligned}
$$

and finally

$$
\left[\mathrm{d} p_{2 ; m}^{(\hat{s}, i r)}\right]=\left[\mathrm{d} p_{1 ; m+1}^{(i r)}\right]\left[\mathrm{d} p_{1 ; m}^{(\hat{s})}\right] \propto\left(1-\alpha_{i r}\right)^{2 m(1-\epsilon)-1}\left(1-y_{\hat{s} Q}\right)^{(m-1)(1-\epsilon)-1} .
$$

The subtraction terms, as originally defined in ref. [22] do not depend on the number of hard partons, thus the $m$-dependence of the factorised phase space measures is carried over to the integrated counterterms, where this dependence enters in a rather cumbersome way (see e.g., eqs. (A.9) and (A.10) of ref. [4]).

Thus, as in refs. $[25,31]$, we reshuffle the $m$-dependence of the integrated counterterms into the subtraction terms themselves, where it appears in a very straightforward and harmless way, ${ }^{7}$ through factors of $(1-\alpha)$ and/or $(1-y)$ raised to $m$-dependent powers. We gather the results in table 20 , where together with the subtraction terms, we give the momentum mappings used to define the term(s) and the expression which multiplies the original counterterm to produce the modified one. The function $f$ in table 20 is defined as

$$
f\left(z_{0}, z, p\right)=\Theta\left(z_{0}-z\right)(1-z)^{-p} .
$$

It is important to note that the functions $d(m, \epsilon), d^{\prime}(m, \epsilon)$ and constants $\alpha_{0}, y_{0}$ in table 20 are the same as those in all other modified subtraction terms, discussed in refs. [25, 31].

The form of the exponents $d(m, \epsilon)$ and $d^{\prime}(m, \epsilon)$ is actually fixed by the prescription adopted in ref. [25] (see in particular eqs. (3.2), (3.12) and (3.13) therein) and the requirement that the modified subtraction terms should still correctly regularise all kinematic singularities. In fact, we must have

$$
d(m, \epsilon)=2 m(1-\epsilon)-2 d_{0}, \quad \text { and } \quad d^{\prime}(m, \epsilon)=m(1-\epsilon)-d_{0}^{\prime},
$$

where $d_{0}$ and $d_{0}^{\prime}$ are the same constants which appear in eqs. (3.2), (3.12) and (3.13) of ref. [25] i.e.,

$$
d_{0}=D_{0}+d_{1} \epsilon, \quad \text { and } \quad d_{0}^{\prime}=D_{0}^{\prime}+d_{1}^{\prime} \epsilon,
$$

where $D_{0}, D_{0}^{\prime} \geq 2$ are integers, while $d_{1}, d_{1}^{\prime}$ are real.

\section{B Spin-averaged splitting kernels}

In this appendix, we list the spin-averaged splitting kernels. Although some of these already appeared elsewhere, for the sake of completeness, we give all the splitting functions of our computations.

\footnotetext{
${ }^{7}$ The modifications introduced above do not spoil any of the cancellations that take place among the original subtraction terms. Hence the modified counterterms are still correct regulators of all kinematic singularities.
} 


\begin{tabular}{|c|c|c|}
\hline \multicolumn{3}{|c|}{ Doubly unresolved counterterms } \\
\hline Subtraction term & Momentum mapping & Function \\
\hline $\mathcal{C}_{\text {irs }}^{(0,0)}$ & $\{p\} \stackrel{\mathcal{C}_{\text {irs }}}{\longrightarrow}\{\tilde{p}\}_{m}^{(\text {irs })}$ & $f\left(\alpha_{0}, \alpha_{i r s}, d(m, \epsilon)\right)$ \\
\hline $\mathcal{C}_{i r ; j s}^{(0,0)}$ & $\{p\} \stackrel{\mathcal{C}_{i r ; j s}}{\longrightarrow}\{\tilde{p}\}_{m}^{(i r ; j s)}$ & $f\left(\alpha_{0}, \alpha_{i r}+\alpha_{j s}, d(m, \epsilon)\right)$ \\
\hline $\begin{array}{c}\mathcal{C} \mathcal{S}_{i r ; s}^{(0,0)}, \mathcal{C}_{i r s} \mathcal{C S}_{i r ; s}^{(0,0)} \\
\mathcal{C}_{i r ; j s} \mathcal{C S}_{i r ; s}^{(0,0)}\end{array}$ & $\{p\} \stackrel{\mathcal{C}_{i r}}{\longrightarrow}\{\hat{p}\}_{m+1}^{(i r)} \stackrel{\mathcal{S}_{\hat{s}}}{\longrightarrow}\{\tilde{p}\}_{m}^{(\hat{s}, i r)}$ & $\begin{array}{c}f\left(\alpha_{0}, \alpha_{i r}, d(m, \epsilon)\right) \\
\times f\left(y_{0}, y_{\hat{s} Q}, d^{\prime}(m, \epsilon)\right)\end{array}$ \\
\hline $\begin{array}{c}\mathcal{S}_{r s}^{(0,0)}, \mathcal{C} \mathcal{S}_{i r ; s} \mathcal{S}_{r s}^{(0,0)} \\
\mathcal{C}_{i r s} \mathcal{S}_{r s}^{(0,0)}, \mathcal{C}_{i r ; j s} \mathcal{S}_{r s}^{(0,0)} \\
\mathcal{C}_{i r s} \mathcal{S}_{i r ; s} \mathcal{S}_{r s}^{(0,0)}\end{array}$ & $\{p\} \stackrel{\mathcal{S}_{r g}}{\longrightarrow}\{\tilde{p}\}_{m}^{(r s)}$ & $f\left(y_{0}, y_{r Q}+y_{s Q}-y_{r s}, d^{\prime}(m, \epsilon)\right)$ \\
\hline
\end{tabular}

Table 20. The modified doubly unresolved subtraction terms are obtained from the original counterterms (first column) by multiplication with an appropriate function (last column). Also shown are the momentum mappings used to define the subtraction terms (middle column).

\section{B.1 Two-parton kernels}

The azimuthally averaged two-parton splitting kernels are well known,

$$
\begin{aligned}
P_{g g}^{(0)}(z) & =2 C_{\mathrm{A}}\left[\frac{1}{z}+\frac{1}{1-z}-2+z-z^{2}\right], \\
P_{q \bar{q}}^{(0)}(z ; \epsilon) & =T_{\mathrm{R}}\left[1-\frac{2}{1-\epsilon}\left(z-z^{2}\right)\right], \\
P_{q g}^{(0)}(z ; \epsilon) & =C_{\mathrm{F}}\left[\frac{2}{z}-2+(1-\epsilon) z\right] .
\end{aligned}
$$

In our convention the ordering of the labels on the splitting kernels is usually meaningless, but in eq. (B.3) $z$ means the momentum fraction of the second label, i.e., $P_{g q}^{(0)}(z ; \epsilon)=$ $P_{q g}^{(0)}(1-z ; \epsilon)$. The other two cases are symmetric with respect to $z \leftrightarrow 1-z$.

\section{B.2 Three-parton kernels}

The spin average of the splitting kernels was computed in ref. [52], however the forms presented there for gluon splittings are not suitable for us, as it was explained in ref. [22]: in the gluon splitting kernels $\hat{P}_{g_{i} q_{r} \bar{q}_{s}}$ and $\hat{P}_{g_{i} g_{r} g_{s}}$, the terms that depend on the transverse momenta must always be written in the form $k_{\perp, j}^{\mu} k_{\perp, k}^{\nu} / k_{\perp, j} \cdot k_{\perp, k}$ ( $k$ can be equal to $j$ ). Otherwise the collinear behaviour of the counterterm cannot be matched with that of the singly collinear counterterm in the singly unresolved region of phase space. The correct azimuth dependence can be achieved by substitutions according to the following replacements,

$$
\begin{aligned}
k_{\perp, j}^{\mu} k_{\perp, j}^{\nu} & \rightarrow\left(-z_{j}\left(1-z_{j}\right) s_{j k l}+z_{j} s_{k l}\right) \frac{k_{\perp, j}^{\mu} k_{\perp, j}^{\nu}}{k_{\perp, j}^{2}}, \\
2 k_{\perp, j}^{\mu} k_{\perp, k}^{\nu} & \rightarrow\left(s_{j k}+2 z_{j} z_{k} s_{j k l}-z_{i} s_{j(i k)}-z_{j} s_{i(j k)}\right) \frac{k_{\perp, j}^{\mu} k_{\perp, k}^{\nu}}{k_{\perp, j} \cdot k_{\perp, k}},
\end{aligned}
$$


where $\{k, l\}=\{i, r, s\} \backslash\{j\}$ and $j$ can be $i, r$ or $s$. With these forms azimuthal averaging amounts to the simple substitutions,

$$
\left\langle\frac{k_{\perp, j}^{\mu} k_{\perp, k}^{\nu}}{k_{\perp, j} \cdot k_{\perp, k}}\right\rangle=-\frac{1}{2(1-\epsilon)},
$$

where $\langle\ldots\rangle$ denote spin averaging, and $j=k$ is also allowed.

For quark splitting into unequal and equal quark flavours we have,

$$
P_{q_{i} \bar{q}_{r}^{\prime} q_{s}^{\prime}}^{(0)}\left(\left\{z_{j, k l}, s_{j k}\right\} ; \epsilon\right)=P_{q_{i} \bar{q}_{r}^{\prime} q_{s}^{\prime}}\left(\left\{z_{j, k l}, s_{j k}\right\} ; \epsilon\right)
$$

and

$$
P_{q_{i} \bar{q}_{r} q_{s}}^{(0)}\left(\left\{z_{j, k l}, s_{j k}\right\} ; \epsilon\right)=2 P_{q_{i} \bar{q}_{r}^{\prime} q_{s}^{\prime}}\left(\left\{z_{j, k l}, s_{j k}\right\} ; \epsilon\right)+P_{q_{i} \bar{q}_{r}^{\prime} q_{s}^{\prime}}^{(\mathrm{id})}\left(\left\{z_{j, k l}, s_{j k}\right\} ; \epsilon\right),
$$

respectively (hence eq. (3.9)), where

$$
\begin{aligned}
\frac{1}{s_{i r s}^{2}} P_{q_{i} \bar{q}_{r}^{\prime} q_{s}^{\prime}}\left(\left\{z_{j, k l}, s_{j k}\right\} ; \epsilon\right)= & C_{\mathrm{F}} T_{\mathrm{R}}\left\{\frac { 1 } { s _ { i r s } s _ { r s } } \left[\frac{z_{i, r s}}{z_{r, i s}+z_{s, i r}}-\frac{s_{i r} z_{s, i r}+s_{i s} z_{r, i s}}{s_{r s}\left(z_{r, i s}+z_{s, i r}\right)}+\frac{s_{i r} s_{i s}}{s_{i r s} s_{r s}}\right.\right. \\
& \left.+\frac{s_{i r s}}{s_{r s}} \frac{z_{r, i s} z_{s, i r}}{\left(z_{r, i s}+z_{s, i r}\right)^{2}}-\frac{z_{r, i s} z_{s, i r}}{z_{r, i s}+z_{s, i r}}+\frac{1-\epsilon}{2}\left(z_{r, i s}+z_{s, i r}-\frac{s_{r s}}{s_{i r s}}\right)\right] \\
& +(r \leftrightarrow s)\}
\end{aligned}
$$

and

$$
\begin{aligned}
\frac{1}{s_{i r s}^{2}} P_{q_{i} \bar{q}_{r}^{\prime} q_{s}^{\prime}}^{(\mathrm{i})}\left(\left\{z_{j, k l}, s_{j k}\right\} ; \epsilon\right)= & C_{\mathrm{F}}\left(C_{\mathrm{F}}-\frac{C_{\mathrm{A}}}{2}\right)\left\{\frac{1-\epsilon}{s_{i r s}^{2}}\left(\frac{2 s_{i s}}{s_{r s}}-\epsilon\right)+\frac{1}{s_{r s} s_{i r s}}\left[\frac{1+z_{r, i s}^{2}}{z_{i, r s}+z_{r, i s}}\right.\right. \\
& -\frac{2 z_{s, i r}}{z_{r, i s}+z_{s, i r}}-\epsilon\left(\frac{\left(z_{r, i s}+z_{s, i r}\right)^{2}}{z_{i, r s}+z_{r, i s}}+1+z_{r, i s}-\frac{2 z_{s, i r}}{z_{r, i s}+z_{s, i r}}\right) \\
& \left.-\epsilon^{2}\left(z_{r, i s}+z_{s, i r}\right)\right]-\frac{1}{s_{i r} s_{r s}} \frac{z_{r, i s}}{2}\left[\frac{1+z_{r, i s}^{2}}{\left(z_{i, r s}+z_{r, i s}\right)\left(z_{r, i s}+z_{s, i r}\right)}\right. \\
& \left.\left.-\epsilon\left(1+2 \frac{z_{i, r s}+z_{r, i s}}{z_{r, i s}+z_{s, i r}}\right)-\epsilon^{2}\right]+(i \leftrightarrow s)\right\} .
\end{aligned}
$$

For splitting into a quark and a gluon pair we find,

$$
P_{q_{i} g_{r} g_{s}}^{(0)}\left(\left\{z_{j, k l}, s_{j k}\right\} ; \epsilon\right)=C_{\mathrm{F}} P_{q, g_{r} g_{s}}^{(\mathrm{ab})}\left(\left\{z_{j, k l}, s_{j k}\right\} ; \epsilon\right)+C_{\mathrm{A}} P_{q_{i} g_{r} g_{s}}^{(\mathrm{nab})}\left(\left\{z_{j, k l}, s_{j k}\right\} ; \epsilon\right),
$$

where

$$
\begin{aligned}
\frac{1}{s_{i r s}^{2}} C_{\mathrm{F}} P_{q_{i} g_{r} g_{s}}^{(\mathrm{ab})}\left(\left\{z_{j, k l}, s_{j k}\right\} ; \epsilon\right)= & C_{\mathrm{F}}^{2}\left\{\left(\frac{1-z_{s, i r}}{s_{i r s} s_{i r}}+\frac{1-z_{r, i s}}{s_{i r s} s_{i s}}+\frac{1-z_{r, i s}-z_{s, i r}}{s_{i r} s_{i s}}\right)\right. \\
& \times\left[\frac{1}{z_{r, i s}}\left(\frac{2}{z_{r, i s}+z_{s, i r}}-2+(1-\epsilon) z_{s, i r}\right)+1-\frac{\epsilon(1+\epsilon)}{2}\right] \\
& +\frac{1-\epsilon}{s_{i r s}^{2}}\left[\epsilon-\frac{s_{i r s}}{s_{i r}}(1+\epsilon)\left(3-z_{r, i s}-2 z_{s, i r}\right)-\frac{s_{i s}}{s_{i r}}(1-\epsilon)\right] \\
& +(r \leftrightarrow s)\}
\end{aligned}
$$


and

$$
\begin{aligned}
& \frac{1}{s_{i r s}^{2}} C_{\mathrm{A}} P_{q_{i} g_{r} g_{s}}^{(\mathrm{nab})}\left(\left\{z_{j, k l}, s_{j k}\right\} ; \epsilon\right)= \\
& C_{\mathrm{A}} C_{\mathrm{F}}\left\{\frac { 1 } { s _ { i r s } s _ { r s } } \left[(1-\epsilon)\left(\frac{s_{i r} z_{s, i r}+s_{i s} z_{r, i s}}{s_{r s}\left(z_{r, i s}+z_{s, i r}\right)}-\frac{s_{i r} s_{i s}}{s_{i r s} s_{r s}}-\frac{s_{i r s}}{s_{r s}} \frac{z_{r, i s} z_{s, i r}}{\left(z_{r, i s}+z_{s, i r}\right)^{2}}\right)\right.\right. \\
& \left.-z_{i, r s}\left(\frac{4}{z_{r, i s}+z_{s, i r}}-\frac{1}{z_{r, i s}}\right)\right]-\frac{1}{s_{i r s} s_{i r}} \frac{\left(1-z_{r, i s}\right)^{2}+\left(1-z_{s, i r}\right)^{2}}{2 z_{r, i s}\left(z_{r, i s}+z_{s, i r}\right)} \\
& -\frac{1}{s_{i r} s_{i s}} \frac{z_{i, r s}}{2 z_{r, i s}} \frac{1+z_{i, r s}^{2}}{z_{r, i s}+z_{s, i r}}+\frac{1}{2 s_{i r} s_{r s}}\left[\frac{1+z_{i, r s}^{2}}{z_{s, i r}}+\frac{1+\left(1-z_{s, i r}\right)^{2}}{z_{r, i s}+z_{s, i r}}\right]+\frac{(1-\epsilon)^{2}}{2 s_{i r s}^{2}} \\
& +\frac{1}{s_{i r s} s_{r s}}\left[(1-\epsilon)\left(\frac{2}{z_{r, i s}}-\frac{1}{z_{r, i s}+z_{s, i r}}\right) \frac{\left(z_{r, i s}-z_{s, i r}\right)^{2}}{4}-1\right]+\frac{1}{2 s_{i r s} s_{i r}} \\
& \times\left[\frac{1+\left(1-z_{s, i r}\right)^{2}}{z_{r, i s}}-\frac{4-2 z_{s, i r}+z_{s, i r}^{2}-z_{r, i s}}{z_{r, i s}+z_{s, i r}}\right]+\frac{\epsilon}{2}\left[\frac { 1 } { s _ { i r s } s _ { i r } } \left(\left(1-z_{s, i r}\right)\right.\right. \\
& \left.\times\left(\frac{z_{r, i s}}{z_{s, i r}}+\frac{z_{s, i r}}{z_{r, i s}}-\epsilon\right)-\frac{z_{r, i s}^{2}\left(1-z_{s, i r}\right)}{z_{s, i r}\left(z_{r, i s}+z_{s, i r}\right)}\right)+\frac{z_{i, r s}}{s_{i r} s_{i s}}\left(\frac{z_{r, i s}}{z_{s, i r}}+\frac{1+\epsilon}{2}\right) \\
& \left.\left.-\frac{1}{s_{i r} s_{r s}}\left(\frac{\left(z_{r, i s}+z_{s, i r}\right)^{2}}{z_{s, i r}}+\frac{z_{s, i r}^{2}}{z_{r, i s}+z_{s, i r}}\right)\right]+(r \leftrightarrow s)\right\} .
\end{aligned}
$$

We call attention to the normalisation (in colour space) of the abelian and non-abelian parts of the splitting function in eq. (B.10), which is the same as in ref. [20]. Notice that a factor of $C_{\mathrm{F}}$, respectively $C_{\mathrm{A}}$, is made explicit in the definition of the abelian, respectively non-abelian, part as compared to the complete splitting function. However, we prefer to define $\left[\mathrm{C}_{i r s}^{(0)}\right]_{f_{i} f_{r} f_{s}}^{(\mathrm{ab})}$ and $\left[\mathrm{C}_{i r s}^{(0)}\right]_{f_{i} f_{r} f_{s}}^{(\mathrm{nab})}$ to be dimensionless in colour space, hence the factors of $C_{\mathrm{F}}$ and $C_{\mathrm{A}}$ are not made explicit in the definition of these functions, see eq. (4.2). Then, eq. (4.1) must be interpreted with some care when computing $\left[\mathrm{C}_{i r s}^{(0)}\right]_{f_{i} f_{r} f_{s}}^{(\mathrm{ab})}$ or $\left[\mathrm{C}_{i r s}^{(0)}\right]_{f_{i} f_{r} f_{s}}^{(\mathrm{nab})}$. In particular, we must remember to include the factors of $C_{\mathrm{F}}$ and $C_{\mathrm{A}}$ explicitly with $P_{f_{i} f_{r} f_{s}}^{(\mathrm{a})}$ and $P_{f_{i} f_{r} f_{s}}^{(\mathrm{nab}}$, i.e., we must set $P_{f_{i} f_{r} f_{s}}^{(0)} \rightarrow C_{\mathrm{F}} P_{f_{i} f_{r} f_{s}}^{(\mathrm{ab})}$ or $P_{f_{i} f_{r} f_{s}}^{(0)} \rightarrow C_{\mathrm{A}} P_{f_{i} f_{r} f_{s}}^{(\mathrm{nab})}$ to obtain the correct normalisation.

For gluon splitting into a gluon and a quark pair we have

$$
P_{g_{i} q_{r} \bar{q}_{s}}^{(0)}\left(\left\{z_{j, k l}, s_{j k}\right\} ; \epsilon\right)=C_{\mathrm{F}} P_{g_{i} q_{r} \bar{q}_{s}}^{(\mathrm{ab})}\left(\left\{z_{j, k l}, s_{j k}\right\} ; \epsilon\right)+C_{\mathrm{A}} P_{g_{i} q_{r} \bar{q}_{s}}^{(\mathrm{nab})}\left(\left\{z_{j, k l}, s_{j k}\right\} ; \epsilon\right),
$$

where

$$
\begin{aligned}
\frac{1}{s_{i r s}^{2}} C_{\mathrm{F}} P_{g_{i} q_{r} \bar{q}_{s}}^{(\mathrm{ab})}\left(\left\{z_{j, k l}, s_{j k}\right\} ; \epsilon\right)= & C_{\mathrm{F}} T_{\mathrm{R}}\left\{\left[\frac{1}{s_{i r} s_{i s}}-\frac{1}{s_{i r s}^{2}}-\frac{2}{s_{i r s} s_{i r}}\left(1-\frac{1-\epsilon}{2} \frac{s_{i r}+s_{i s}}{s_{i r s}}\right)\right]\right. \\
& -\frac{1}{1-\epsilon} \frac{1}{s_{i r s} s_{i r} s_{i s}}\left[2 z_{r, i s} z_{s, i r} s_{i r s}+z_{i, r s} s_{r s}-z_{r, i s} s_{i s}-z_{s, i r} s_{i r}\right. \\
& \left.\left.-(1-\epsilon)\left(z_{i, r s} s_{r s}-z_{i, r s}\left(1-z_{i, r s}\right) s_{i r s}\right)\right]+(r \leftrightarrow s)\right\} \quad \text { (B.14) }
\end{aligned}
$$


and

$$
\begin{aligned}
& \frac{1}{s_{i r s}^{2}} C_{\mathrm{A}} P_{g_{i} q_{r} \bar{q}_{s}}^{(\mathrm{nab}}\left(\left\{z_{j, k l}, s_{j k}\right\} ; \epsilon\right)= \\
& C_{\mathrm{A}} T_{\mathrm{R}}\left\{\frac { 1 } { s _ { i r s } s _ { r s } } \left(\frac{1}{z_{r, i s}+z_{s, i r}}-1-\frac{s_{i r} z_{s, i r}+s_{i s} z_{r, i s}}{s_{r s}\left(z_{r, i s}+z_{s, i r}\right)}+\frac{s_{i r} s_{i s}}{s_{r s} s_{i r s}}\right.\right. \\
& \left.+\frac{s_{i r s}}{s_{r s}} \frac{z_{r, i s} z_{s, i r}}{\left(z_{r, i s}+z_{s, i r}\right)^{2}}+\frac{1}{2 z_{i, r s}}\right)+\frac{1}{s_{i r} s_{r s}}\left(\frac{z_{r, i s}}{2 z_{i, r s}}-\frac{z_{r, i s}}{2\left(z_{r, i s}+z_{s, i r}\right)}\right) \\
& +\frac{1}{s_{i r s} s_{i s}} \frac{1-z_{r, i s}}{2}\left(\frac{1}{z_{i, r s}}+\frac{1}{z_{r, i s}+z_{s, i r}}\right)-\frac{1}{2 s_{i r} s_{i s}}-\frac{1-\epsilon}{2 s_{i r s}^{2}} \\
& -\frac{1}{s_{i r s} s_{r s}} \frac{1}{1-\epsilon} \frac{2 z_{r, i s} z_{s, i r}}{z_{i, r s}\left(z_{r, i s}+z_{s, i r}\right)}-\frac{1}{s_{i r s} s_{i r} s_{r s}}\left[z_{r, i s} s_{i s}-z_{r, i s}\left(1-z_{r, i s}\right) s_{i r s}\right. \\
& -\frac{1}{1-\epsilon} \frac{2 z_{r, i s}^{2}}{z_{i, r s}\left(z_{r, i s}+z_{s, i r}\right)}\left(z_{s, i r} s_{i r}-z_{s, i r}\left(1-z_{s, i r}\right) s_{i r s}\right)+\left(\frac{2 z_{r, i s}\left(z_{s, i r}-z_{i, r s}\right)}{z_{i, r s}\left(z_{r, i s}+z_{s, i r}\right)}\right. \\
& \left.+1-\epsilon) \frac{1}{2(1-\epsilon)}\left(2 z_{r, i s} z_{s, i r} s_{i r s}+z_{i, r s} s_{r s}-z_{r, i s} s_{i s}-z_{s, i r} s_{i r}\right)\right]+\frac{1}{s_{i r s} s_{i r} s_{i s}} \\
& \quad \times\left[\left(2 z_{r, i s} z_{s, i r} s_{i r s}+z_{i, r s} s_{r s}-z_{r, i s} s_{i s}-z_{s, i r} s_{i r}\right) \frac{1}{2(1-\epsilon)}-\frac{1}{2}\left(z_{i, r s} s_{r s}\right.\right. \\
& \left.\left.\left.-z_{i, r s}\left(1-z_{i, r s}\right) s_{i r s}\right)\right]+(r \leftrightarrow s)\right\} .
\end{aligned}
$$

Note again that the normalisation (in colour space) of the abelian and non-abelian parts of the splitting function in eq. (B.13) matches that of ref. [20], i.e., a factor of $C_{\mathrm{F}}$ and $C_{\mathrm{A}}$ is made explicit in the definition of the abelian and non-abelian piece respectively. Hence, the comments below eq. (B.12) apply in this case as well.

Finally for splitting into three gluons we have

$$
\begin{aligned}
& \frac{1}{s_{i r s}^{2}} P_{g_{i} g_{r} g_{s}}^{(0)}\left(\left\{z_{j, k l}, s_{j k}\right\} ; \epsilon\right)= \\
& C_{\mathrm{A}}^{2} \frac{1}{s_{i r s}^{2}}\left\{\frac{(1-\epsilon)}{4 s_{r s}^{2}}\left(2 \frac{s_{i s} z_{r, i s}-s_{i r} z_{s, i r}}{z_{r, i s}+z_{s, i r}}+\frac{z_{r, i s}-z_{s, i r}}{z_{r, i s}+z_{s, i r}} s_{r s}\right)^{2}+\frac{3}{4}(1-\epsilon)\right. \\
& +\frac{2 s_{i r s} z_{r, i s} z_{s, i r}}{s_{r s} z_{i, r s}\left(1-z_{i, r s}\right)}-\frac{s_{i r s}}{s_{r s}} \frac{1}{z_{i, r s}}\left[\frac{2\left(1-z_{i, r s}\right)+4 z_{i, r s}^{2}}{1-z_{i, r s}}-\frac{1-2 z_{i, r s}\left(1-z_{i, r s}\right)}{z_{r, i s}\left(1-z_{r, i s}\right)}\right] \\
& +\frac{s_{i r s}}{s_{i r} s_{r s}}\left[z_{r, i s} \frac{s_{i r s} z_{i, r s}\left(1-z_{i, r s}\right)-s_{r s} z_{i, r s}}{z_{s, i r}\left(1-z_{s, i r}\right)}\left(1-2 z_{s, i r}\right)\right. \\
& +z_{r, i s} \frac{s_{i r s} z_{s, i r}\left(1-z_{s, i r}\right)-s_{i r} z_{s, i r}}{z_{i, r s}\left(1-z_{i, r s}\right)}\left(1-2 z_{i, r s}\right) \\
& -\frac{s_{i r s}}{2}\left(\frac{4 z_{i, r s} z_{s, i r}+2 z_{r, i s}\left(1-z_{r, i s}\right)-1}{\left(1-z_{i, r s}\right)\left(1-z_{s, i r}\right)}-\frac{1-2 z_{r, i s}\left(1-z_{r, i s}\right)}{z_{i, r s} z_{s, i r}}\right) \\
& \left.-\left(\frac{2 z_{s, i r}\left(1-z_{s, i r}\right)}{z_{i, r s}\left(1-z_{i, r s}\right)}-3\right) \frac{2 s_{i r s} z_{i, r s} z_{s, i r}-s_{i r} z_{s, i r}-s_{r s} z_{i, r s}+s_{i s} z_{r, i s}}{2}\right] \\
& +(5 \text { permutations })\} .
\end{aligned}
$$




\section{Integrating the triple collinear counterterm}

\section{C.1 Master integrals}

The triple collinear momentum mapping leads to the exact factorisation of phase space as given by eq. (2.12), where the two-particle factorised phase space can be written as

$$
\begin{aligned}
{\left[\mathrm{d} p_{2 ; m}^{(i r s)}\left(p_{r}, p_{s}, \tilde{p}_{i r s} ; Q\right)\right]=} & \mathrm{d} \alpha(1-\alpha)^{2(m-1)(1-\epsilon)-1} \frac{s \widetilde{\text { irs } Q}}{2 \pi} \mathrm{d} \phi_{3}\left(p_{i}, p_{r}, p_{s} ; p_{(i r s)}\right) \\
& \times \Theta(\alpha) \Theta(1-\alpha),
\end{aligned}
$$

with $p_{(i r s)}^{\mu}=(1-\alpha) \tilde{p}_{i r s}^{\mu}+\alpha Q^{\mu}$. When writing eq. (4.1), we have used the fact that the spin correlations present at the level of the factorisation formula vanish upon azimuthal integration via the usual arguments, since $k_{\perp, j, k}^{\mu}(j, k=i, r$ and $s)$ as defined in ref. [22] is orthogonal to $\tilde{p}_{i r s}^{\mu}$. Therefore, the integrals of the spin-dependent and spin-averaged splitting functions are equal.

The spin-averaged triple collinear functions $P_{f_{i} f_{r} f_{s}}^{(0)}$ depend on six variables: three twoparticle invariants: $s_{i r}, s_{i s}, s_{r s}$ and three momentum fractions: $z_{i, r s}, z_{r, i s}$ and $z_{s, i r}$, and are given in appendix B. When counting the number of independent kinematical structures in eqs. (B.8)-(B.16), we make two observations. Firstly, of the six variables, only four are independent, since $s_{i r}+s_{i s}+s_{r s}=s_{i r s}$ and $z_{i, r s}+z_{r, i s}+z_{s, i r}=1$. Secondly, from eq. (C.1) it is clear that the factorised phase-space measure $\left[\mathrm{d} p_{2 ; m}^{(i r s)}\left(p_{r}, p_{s}, \tilde{p}_{i r s} ; Q\right)\right]$ is fully symmetric under permutations of the indices $\{i, r, s\}$. This permutation symmetry relates integrals of different terms,

$$
\int_{2}\left[\mathrm{~d} p_{2 ; m}^{(i r s)}\left(p_{r}, p_{s}, \tilde{p}_{i r s} ; Q\right)\right] P\left(p_{i}, p_{r}, p_{s}\right)=\int_{2}\left[\mathrm{~d} p_{2 ; m}^{(i r s)}\left(p_{r}, p_{s}, \tilde{p}_{i r s} ; Q\right)\right] P\left(p_{\sigma(i)}, p_{\sigma(r)}, p_{\sigma(s)}\right),
$$

where $\sigma \in S_{3}$, with $P$ an arbitrary function of momenta (a term in the splitting functions). This further reduces the number of independent structures.

Making use of the constraints among variables and the $S_{3}$ permutation symmetry, we find 46 structures to integrate, ${ }^{8}$ that can be grouped into five classes,

$$
\begin{aligned}
& P_{1}^{(j, k, l, m)}=t_{i r}^{j} t_{i s}^{k} t_{r s}^{l} z_{r, i s}^{m}, \\
& P_{2}^{(j, k, l, m)}=t_{i r}^{j} t_{i s}^{k} z_{r, i s}^{l} z_{s, i r}^{m}, \\
& P_{3}^{(j, k, l, m)}=t_{i r}^{j} t_{i s}^{k} z_{s, i r}^{l}\left(1-z_{r, i s}\right)^{m}, \\
& P_{4}^{(j, k, l, m)}=t_{i r}^{j} t_{r s}^{k}\left(1-z_{r, i s}\right)^{l} z_{s, i r}^{m}, \\
& P_{5}^{(j, k, l, m)}=t_{i s}^{j} t_{r s}^{k}\left(1-z_{r, i s}\right)^{l}\left(1+z_{s, i r}\right)^{m},
\end{aligned}
$$

where we introduced the scaled two-particle invariants $t_{k l}=s_{k l} / s_{i r s}(k, l=i, r$, or $s)$. The exponents take values as given in tables $1-5$. With these five classes, we can give new forms of the spin-averaged splitting functions that lead to the same integrated triple collinear subtraction terms,

$$
\frac{1}{s_{i r s}^{2}} \frac{1}{\left(\boldsymbol{T}_{i r s}^{2}\right)^{2}} P_{f_{i} f_{r} f_{s}}^{(0)} \rightarrow a_{f_{i} f_{r} f_{s}} \sum_{n=1}^{5} \sum_{j, k, l, m} c_{f_{i} f_{r} f_{s} ; n}^{(0), j, k, l, m} P_{n}^{(j, k, l, m)},
$$

\footnotetext{
${ }^{8}$ This basic set of integrals is not unique, nor do we claim that they are linearly independent.
} 
where the constants $a_{f_{i} f_{r} f_{s}}$ are given in eq. (4.4), while $c_{f_{i} f_{r} f_{s} ; i}^{(0), j, k}$ in tables 1-5. Thus we are left with five types of integrals,

$$
\begin{aligned}
\mathcal{I}_{2 \mathcal{C}, n}^{(j, k, l, m)}\left(x_{\overparen{i r s}}, \epsilon ; \alpha_{0}, d_{0}\right)=\left(\frac{(4 \pi)^{2}}{S_{\epsilon}} Q^{2 \epsilon}\right)^{2} & \int_{2}\left[\mathrm{~d} p_{2 ; m}^{(i r s)}\left(p_{r}, p_{s}, \tilde{p}_{i r s} ; Q\right)\right] \frac{1}{s_{i r s}^{2}} P_{n}^{(j, k, l, m)} \\
& \times f\left(\alpha_{0}, \alpha, d(m, \epsilon)\right), \quad(n=1, \ldots, 5) .
\end{aligned}
$$

\section{C.2 Explicit representations}

In order to write the integrals in eq. (C.5) explicitly, we must choose a specific parametrisation of the factorised phase-space measure. To begin, we choose the scaled two-particle invariants $t_{k l} \equiv s_{k l} / s_{i r s},(k, l=i, r$ or $s)$, and $v_{r}, v_{s}$, defined by

$$
v_{r}=\frac{z_{r, i s}-z_{r}^{(-)}}{z_{r}^{(+)}-z_{r}^{(-)}}, \quad v_{s}=\frac{z_{s, i r}-z_{s}^{(-)}}{z_{s}^{(+)}-z_{s}^{(-)}},
$$

as integration variables. The momentum fractions take values between

$$
z_{r}^{(+)}=\left(1-t_{i s}\right) \frac{\alpha+(1-\alpha) x_{\widetilde{i r s}}}{2 \alpha+(1-\alpha) x_{\widetilde{i r s}}}, \quad z_{r}^{(-)}=\left(1-t_{i s}\right) \frac{\alpha}{2 \alpha+(1-\alpha) x_{\overparen{i r s}}}
$$

and similarly for $z_{s}^{( \pm)}$with $r \leftrightarrow s$. Clearly, $v_{r}$ and $v_{s}$ are simply momentum fractions rescaled to take values between zero and one. In terms of these variables, we have

$$
z_{k, i l}=\left(1-t_{i l}\right) \frac{\alpha+(1-\alpha) x \widetilde{i r s} v_{k}}{2 \alpha+(1-\alpha) x_{\overparen{i r s}}}, \quad k, l=r, s .
$$

Using the variables $t_{k l}$ and $v_{k}$, the factorised phase-space measure reads

$$
\begin{aligned}
{\left[\mathrm{d} p_{2 ; m}^{(i r s)}\left(p_{r}, p_{s}, \tilde{p}_{i r s} ; Q\right)\right]=} & \left(\frac{S_{\epsilon}}{(4 \pi)^{2}} Q^{-2 \epsilon}\right)^{2} \frac{\Gamma^{2}(1-\epsilon)}{\pi \Gamma(1-2 \epsilon)}\left(Q^{2}\right)^{2} x_{\text {irs }} \mathrm{d} \alpha(1-\alpha)^{2(m-1)(1-\epsilon)-1} \\
& \times y_{i r s}^{1-2 \epsilon} \mathrm{d} t_{i r} \mathrm{~d} t_{i s} \mathrm{~d} t_{r s} \mathrm{~d} v_{r} \mathrm{~d} v_{s} \mathrm{~d} y_{i r s} \delta\left(\alpha\left(\alpha+(1-\alpha) x_{\text {irs }}\right)-y_{i r s}\right) \\
& \times \delta\left(1-t_{i r}-t_{i s}-t_{r s}\right)\left(1-t_{i r}\right)\left(1-t_{i s}\right)\left[\left(t_{r s}^{(+)}-t_{r s}\right)\left(t_{r s}-t_{r s}^{(-)}\right)\right]^{-\frac{1}{2}-\epsilon} \\
& \times \Theta\left(1-t_{i r}\right) \Theta\left(t_{i r}\right) \Theta\left(1-t_{i s}\right) \Theta\left(t_{i s}\right) \Theta\left(t_{r s}^{(+)}-t_{r s}\right) \Theta\left(t_{r s}-t_{r s}^{(-)}\right) \\
& \times \Theta\left(1-v_{r}\right) \Theta\left(v_{r}\right) \Theta\left(1-v_{s}\right) \Theta\left(v_{s}\right),
\end{aligned}
$$

where

$$
t_{r s}^{( \pm)}=\left(1-t_{i r}\right)\left(1-t_{i s}\right) \tau^{( \pm)} \quad \text { with } \quad \tau^{( \pm)}=\left[\sqrt{v_{r}\left(1-v_{s}\right)} \pm \sqrt{v_{s}\left(1-v_{r}\right)}\right]^{2} .
$$

Eq. (C.9) is not yet in a very useful form for computing the integrals because it contains constraints in the form of nontrivial $\Theta$ functions. In order to isolate the singular behaviour we map the region of integration onto the unit hypercube such that physical singularities are on the borders. Solving the constraints in a particular way, we find a parametrisation over the unit hypercube where all physical singularities are on the border except $z_{s}=0$, 
which introduces a line singularity (see below). We find

$$
\begin{aligned}
{\left[\mathrm{d} p_{2 ; m}^{(i r s)}\left(p_{r}, p_{s}, \tilde{p}_{i r s} ; Q\right)\right]=} & 2^{-4 \epsilon}\left(\frac{S_{\epsilon}}{(4 \pi)^{2}} Q^{-2 \epsilon}\right)^{2} \frac{\Gamma^{2}(1-\epsilon)}{\pi \Gamma(1-2 \epsilon)}\left(Q^{2}\right)^{2} x_{\overparen{i r s}} \mathrm{~d} \alpha(1-\alpha)^{2(m-1)(1-\epsilon)-1} \\
& \times \mathrm{d} t_{i s} \mathrm{~d} \tau_{r s} \mathrm{~d} v_{r} \mathrm{~d} w_{s} \mathrm{~d} y_{i r s} \delta\left(\alpha\left(\alpha+(1-\alpha) x_{\overparen{i r s}}\right)-y_{i r s}\right) \\
& \times y_{i r s}^{1-2 \epsilon}\left[t_{i s}\left(1-t_{i s}\right)\right]^{1-2 \epsilon}\left[\tau_{r s}\left(1-\tau_{r s}\right) v_{r}\left(1-v_{r}\right)\right]^{-\epsilon} \\
& \times\left[w_{s}\left(1-w_{s}\right)\right]^{-\frac{1}{2}-\epsilon}\left(1-\tau_{r s}+\tau_{r s} t_{i s}\right)^{-2+2 \epsilon} \\
& \times \Theta\left(1-t_{i s}\right) \Theta\left(t_{i s}\right) \Theta\left(1-\tau_{r s}\right) \Theta\left(\tau_{r s}\right) \\
& \times \Theta\left(1-v_{r}\right) \Theta\left(v_{r}\right) \Theta\left(1-w_{s}\right) \Theta\left(w_{s}\right) .
\end{aligned}
$$

In terms of the variables $t_{i s}, \tau_{r s}, v_{r}$ and $w_{s}$ we have

$$
t_{i r}=\frac{\left(1-\tau_{r s}\right)\left(1-t_{i s}\right)}{1-\tau_{r s}+\tau_{r s} t_{i s}}, \quad t_{r s}=\frac{\tau_{r s} t_{i s}\left(1-t_{i s}\right)}{1-\tau_{r s}+\tau_{r s} t_{i s}}
$$

and

$$
\begin{aligned}
z_{r, i s}= & \left(1-t_{i s}\right) \frac{\alpha+(1-\alpha) x_{\overparen{i r s}} v_{r}}{2 \alpha+(1-\alpha) x_{\overparen{i r s}}}, \\
z_{s, i r}= & \frac{t_{i s}}{1-\tau_{r s}+\tau_{r s} t_{i s}} \\
& \times \frac{\alpha+(1-\alpha) x_{\overparen{i r s}}\left[\tau_{r s}\left(1-v_{r}\right)+v_{r}\left(1-\tau_{r s}\right)-2 \sqrt{\tau_{r s}\left(1-\tau_{r s}\right) v_{r}\left(1-v_{r}\right)}\left(1-2 w_{s}\right)\right]}{2 \alpha+(1-\alpha) x_{\overparen{i r s}}} .
\end{aligned}
$$

We see that $z_{s}=0$ corresponds to $\alpha=0, w_{s}=0,1$ and $\tau_{r s}=v_{r}$, hence the line singularity. Since the only integral involving $1 / z_{s}$ is $\mathcal{I}_{2 \mathcal{C}, 4}$, this is the only place where the line singularity has to be resolved.

Using the parametrisation of eq. (C.11) and eqs. (C.12) and (C.13), we find the following explicit parametric integral representations of the basic integrals.

$$
\begin{aligned}
& \mathcal{I}_{2 \mathcal{C}, 1}^{(j, k, l, m)}\left(x_{\overparen{i r s}}, \epsilon ; \alpha_{0}, d_{0}\right)=2^{-4 \epsilon} \frac{\Gamma^{2}(1-\epsilon)}{\pi \Gamma(1-2 \epsilon)} x_{\widetilde{i r s}} \int_{0}^{\alpha_{0}} \mathrm{~d} \alpha \int_{0}^{1} \mathrm{~d} t_{i s} \mathrm{~d} \tau_{r s} \mathrm{~d} v_{r} \mathrm{~d} w_{s} \\
& \times \alpha^{-1-2 \epsilon}(1-\alpha)^{2 d_{0}-3+2 \epsilon}\left(\alpha+(1-\alpha) x_{\overparen{i r s}}\right)^{-1-2 \epsilon}\left(2 \alpha+(1-\alpha) x_{\overparen{i r s}}\right)^{-m} \\
& \times t_{i s}^{k+l+1-2 \epsilon}\left(1-t_{i s}\right)^{j+l+m+1-2 \epsilon} \tau_{r s}^{l-\epsilon}\left(1-\tau_{r s}\right)^{j-\epsilon} v_{r}^{-\epsilon}\left(1-v_{r}\right)^{-\epsilon} \\
& \times w_{s}^{-\frac{1}{2}-\epsilon}\left(1-w_{s}\right)^{-\frac{1}{2}-\epsilon}\left(1-\tau_{r s}+\tau_{r s} t_{i s}\right)^{-j-l-2+2 \epsilon}\left(\alpha+(1-\alpha) x_{\overparen{i r s}} v_{r}\right)^{m}, \\
& \mathcal{I}_{2 \mathcal{C}, 2}^{(j, k,-1, m)}\left(x_{\overparen{i r s}}, \epsilon ; \alpha_{0}, d_{0}\right)=2^{-4 \epsilon} \frac{\Gamma^{2}(1-\epsilon)}{\pi \Gamma(1-2 \epsilon)} x_{\overparen{i r s}} \int_{0}^{\alpha_{0}} \mathrm{~d} \alpha \int_{0}^{1} \mathrm{~d} t_{i s} \mathrm{~d} \tau_{r s} \mathrm{~d} v_{r} \mathrm{~d} w_{s} \\
& \times \alpha^{-1-2 \epsilon}(1-\alpha)^{2 d_{0}-3+2 \epsilon}\left(\alpha+(1-\alpha) x_{\widetilde{\text { irs }}}\right)^{-1-2 \epsilon}\left(2 \alpha+(1-\alpha) x_{\overparen{\text { irs }}}\right)^{1-m} \\
& \times t_{i s}^{k+m+1-2 \epsilon}\left(1-t_{i s}\right)^{j-2 \epsilon} \tau_{r s}^{-\epsilon}\left(1-\tau_{r s}\right)^{j-\epsilon} v_{r}^{-\epsilon}\left(1-v_{r}\right)^{-\epsilon} \\
& \times w_{s}^{-\frac{1}{2}-\epsilon}\left(1-w_{s}\right)^{-\frac{1}{2}-\epsilon}\left(1-\tau_{r s}+\tau_{r s} t_{i s}\right)^{-j-m-2+2 \epsilon}\left(\alpha+(1-\alpha) x_{\overparen{i r s}} v_{r}\right)^{-1} \\
& \times\left\{\alpha+(1-\alpha) x_{\overparen{i r s}}\left[\left(1-\tau_{r s}\right) v_{r}+\tau_{r s}\left(1-v_{r}\right)-2 \sqrt{\tau_{r s}\left(1-\tau_{r s}\right) v_{r}\left(1-v_{r}\right)}\left(1-2 w_{s}\right)\right]\right\}^{m},
\end{aligned}
$$




$$
\begin{aligned}
& \mathcal{I}_{2 \mathcal{C}, 3}^{(j, k, l, m)}\left(x_{\overparen{i r s}}, \epsilon ; \alpha_{0}, d_{0}\right)=2^{-4 \epsilon} \frac{\Gamma^{2}(1-\epsilon)}{\pi \Gamma(1-2 \epsilon)} x_{\overparen{i r s}} \int_{0}^{\alpha_{0}} \mathrm{~d} \alpha \int_{0}^{1} \mathrm{~d} t_{i s} \mathrm{~d} \tau_{r s} \mathrm{~d} v_{r} \mathrm{~d} w_{s} \\
& \quad \times \alpha^{-1-2 \epsilon}(1-\alpha)^{2 d_{0}-3+2 \epsilon}\left(\alpha+(1-\alpha) x_{\overparen{i r s}}\right)^{-1-2 \epsilon}\left(2 \alpha+(1-\alpha) x_{\overparen{i r s}}\right)^{-m-l} \\
& \quad \times t_{i s}^{k+l+1-2 \epsilon}\left(1-t_{i s}\right)^{j+1-2 \epsilon} \tau_{r s}^{-\epsilon}\left(1-\tau_{r s}\right)^{j-\epsilon} v_{r}^{-\epsilon}\left(1-v_{r}\right)^{-\epsilon} \\
& \quad \times w_{s}^{-\frac{1}{2}-\epsilon}\left(1-w_{s}\right)^{-\frac{1}{2}-\epsilon}\left(1-\tau_{r s}+\tau_{r s} t_{i s}\right)^{-j-l-2+2 \epsilon} \\
& \quad \times\left[\left(1+t_{i s}\right) \alpha+(1-\alpha) x_{\overparen{i r s}}\left(1-\left(1-t_{i s}\right) v_{r}\right)\right]^{m} \\
& \quad \times\left\{\alpha+(1-\alpha) x_{\overparen{i r s}}\left[\left(1-\tau_{r s}\right) v_{r}+\tau_{r s}\left(1-v_{r}\right)-2 \sqrt{\tau_{r s}\left(1-\tau_{r s}\right) v_{r}\left(1-v_{r}\right)}\left(1-2 w_{s}\right)\right]\right\}^{l}, \\
& \mathcal{I}_{2 \mathcal{C}, 4}^{(j, k,-1,-1)}\left(x \widetilde{i r s}, \epsilon ; \alpha_{0}, d_{0}\right)=2^{-4 \epsilon} \frac{\Gamma^{2}(1-\epsilon)}{\pi \Gamma(1-2 \epsilon)} x \widetilde{i r s} \int_{0}^{\alpha} \mathrm{d} \alpha \int_{0}^{1} \mathrm{~d} t_{i s} \mathrm{~d} \tau_{r s} \mathrm{~d} v_{r} \mathrm{~d} w_{s} \\
& \quad \times \alpha^{-1-2 \epsilon}(1-\alpha)^{2 d_{0}-3+2 \epsilon}\left(\alpha+(1-\alpha) x_{\overparen{i r s}}\right)^{-1-2 \epsilon}\left(2 \alpha+(1-\alpha) x_{\overparen{i r s}}\right)^{2} \\
& \quad \times t_{i s}^{k-2 \epsilon}\left(1-t_{i s}\right)^{j+k+1-2 \epsilon} \tau_{r s}^{k-\epsilon}\left(1-\tau_{r s}\right)^{j-\epsilon} v_{r}^{-\epsilon}\left(1-v_{r}\right)^{-\epsilon} \\
& \quad \times w_{s}^{-\frac{1}{2}-\epsilon}\left(1-w_{s}\right)^{-\frac{1}{2}-\epsilon}\left(1-\tau_{r s}+\tau_{r s} t_{i s}\right)^{-j-k-1+2 \epsilon} \\
& \quad \times\left[\left(1+t_{i s}\right) \alpha+(1-\alpha) x_{\overparen{i r s}}\left(1-\left(1-t_{i s}\right) v_{r}\right)\right]^{-1} \\
& \quad \times\left\{\alpha+(1-\alpha) x_{\overparen{i r s}}\left[\left(1-\tau_{r s}\right) v_{r}+\tau_{r s}\left(1-v_{r}\right)-2 \sqrt{\tau_{r s}\left(1-\tau_{r s}\right) v_{r}\left(1-v_{r}\right)}\left(1-2 w_{s}\right)\right]\right\}^{-1}
\end{aligned}
$$

and

$$
\begin{aligned}
\mathcal{I}_{2 \mathcal{C}, 5}^{(-1,-1,-1,-1)}\left(x_{\overparen{i r s}}, \epsilon ; \alpha_{0}, d_{0}\right)=2^{1-4 \epsilon} \frac{\Gamma^{2}(1-\epsilon)}{\pi \Gamma(1-2 \epsilon)} x_{\widetilde{i r s}} \int_{0}^{\alpha_{0}} \mathrm{~d} \alpha \int_{0}^{1} \mathrm{~d} t_{i s} \mathrm{~d} \tau_{r s} \mathrm{~d} v_{r} \mathrm{~d} w_{s} \\
\quad \times \alpha^{-1-2 \epsilon}(1-\alpha)^{2 d_{0}-3+2 \epsilon}\left(\alpha+(1-\alpha) x_{\widetilde{i r s}}\right)^{-1-2 \epsilon}\left(2 \alpha+(1-\alpha) x_{\overparen{i r s}}\right) \\
\quad \times t_{i s}^{-1-2 \epsilon}\left(1-t_{i s}\right)^{-2 \epsilon} \tau_{r s}^{-1-\epsilon}\left(1-\tau_{r s}\right)^{-\epsilon} v_{r}^{-\epsilon}\left(1-v_{r}\right)^{-\epsilon} w_{s}^{-\frac{1}{2}-\epsilon}\left(1-w_{s}\right)^{-\frac{1}{2}-\epsilon} \\
\quad \times\left(1-\tau_{r s}+\tau_{r s} t_{i s}\right)^{-1+2 \epsilon}\left[\left(1+t_{i s}\right) \alpha+(1-\alpha) x_{\overparen{i r s}}\left(1-\left(1-t_{i s}\right) v_{r}\right)\right]^{-1} \\
\quad \times\left\{1+\frac{t_{i s}}{1-\tau_{r s}+\tau_{r s} t_{i s}}\right. \\
\left.\quad \times \frac{\alpha+(1-\alpha) x_{\overparen{i r s}}\left[\left(1-\tau_{r s}\right) v_{r}+\tau_{r s}\left(1-v_{r}\right)-2 \sqrt{\tau_{r s}\left(1-\tau_{r s}\right) v_{r}\left(1-v_{r}\right)}\left(1-2 w_{s}\right)\right]}{2 \alpha+(1-\alpha) x_{\overparen{i r s}}}\right\}^{-1} .
\end{aligned}
$$

The expressions for $\mathcal{I}_{2 \mathcal{C}, n}, n=1,2$ and 3 in eqs. (C.14)-(C.16) are suitable for evaluation with general purpose sector decomposition codes as they stand. In $\mathcal{I}_{2 C, 5}$, eq. (C.18), the terms in the braces require some care. Although this factor is just $1 /\left(1+z_{s}\right)$, which is finite and hence in principle it can be simply carried through sector decomposition as it is, nevertheless depending on the precise internal implementation, it may lead to undefined arithmetic expressions in the (symbolic) computation. We find that no such trouble arises either with our private implementation or with $\mathrm{SecDec}$, if we gather the two terms in the braces over a common denominator.

$\mathcal{I}_{2 \mathcal{C}, 4}$ requires special attention, as could be anticipated by the presence of the factor $1 / z_{s}$. There are two problems: firstly, the presence of the square roots in the vanishing denominator in the braces on the last line of eq. (C.17) interferes with the treatment of 
overlapping singularities. (This could be solved by simply making the expression squarefree.) Secondly, as we have indicated, there is a line singularity inside the integration region at $\tau_{r s}=v_{r}$. We address both issues by deriving an alternative representation for $\mathcal{I}_{2 \mathcal{C}, 4}$, which is free of both square roots and line singularities. The price to pay is that the new representation is quite cumbersome,

$$
\begin{aligned}
\mathcal{I}_{2 \mathcal{C}, 4}^{(j, k,-1,-1)}\left(x_{\overparen{i r s}}, \epsilon ; \alpha_{0}, d_{0}\right)=-2 \epsilon \frac{\Gamma^{2}(1-\epsilon)}{\Gamma(1-2 \epsilon)} x_{\overparen{i r s}} \int_{0}^{\alpha_{0}} \mathrm{~d} \alpha \int_{0}^{1} \mathrm{~d} t_{i s} \mathrm{~d} \tau_{r s} \mathrm{~d} s \mathrm{~d} u \alpha^{-1-2 \epsilon} \\
\times(1-\alpha)^{2 d_{0}-3+2 \epsilon}\left(\alpha+(1-\alpha) x_{\overparen{i r s}}\right)^{-1-2 \epsilon}\left(2 \alpha+(1-\alpha) x_{\overparen{i r s}}\right)^{4-2 \epsilon} t_{i s}^{k-2 \epsilon} \\
\times\left(1-t_{i s}\right)^{j+k+1-2 \epsilon} \tau_{r s}^{k-\epsilon}\left(1-\tau_{r s}\right)^{j-\epsilon} s^{-2 \epsilon} u^{-2 \epsilon}\left(1-\tau_{r s}+\tau_{r s} t_{i s}\right)^{-j-k-1+2 \epsilon} \\
\times\left[2\left(1+t_{i s}\right) \alpha\left(\alpha+(1-\alpha) x_{\overparen{i r s}}\right)(1+u)\right. \\
\left.\quad+(1-\alpha)^{2}\left(1-\tau_{r s}+\tau_{r s} u\right) x_{\overparen{i r s}}^{2}+t_{i s}(1-\alpha)^{2}\left(\tau_{r s}+u-\tau_{r s} u\right) x_{\overparen{i r s}}^{2}\right]^{1-2 \epsilon} \\
\times\left\{\left[\left(1+t_{i s}\right)^{2} \alpha\left(\alpha+(1-\alpha) x_{\overparen{i r s}}\right)+t_{i s}(1-\alpha)^{2} x_{\widetilde{i r s}}^{2}\right]\left(2 \alpha+(1-\alpha) x_{\overparen{i r s}}\right)^{4}(1-s)^{2} u^{2}\right. \\
\quad+\left[\alpha\left(\alpha+(1-\alpha) x_{\widetilde{i r s}}\right) s(1-u)^{2}+\left(2 \alpha+(1-\alpha) x_{\overparen{i r s}}\right)^{2} u\right] \\
\quad \times s\left[2\left(1+t_{i s}\right) \alpha\left(\alpha+(1-\alpha) x_{\overparen{i r s}}\right)(1+u)\right. \\
\left.\left.\quad+(1-\alpha)^{2}\left(1-\tau_{r s}+\tau_{r s} u\right) x_{\overparen{i r s}}^{2}+t_{i s}(1-\alpha)^{2}\left(\tau_{r s}+u-\tau_{r s} u\right) x_{\overparen{i r s}}^{2}\right]^{2}\right\}^{-1+\epsilon} .
\end{aligned}
$$

The derivation of this alternate form relies on rewriting the integration over $v_{r}$ and $w_{s}$,

$$
\begin{aligned}
I_{v_{r}, w_{s}}= & \int_{0}^{1} \mathrm{~d} v_{r} \mathrm{~d} w_{s} v_{r}^{-\epsilon}\left(1-v_{r}\right)^{-\epsilon} w_{s}^{-\frac{1}{2}-\epsilon}\left(1-w_{s}\right)^{-\frac{1}{2}-\epsilon} \\
& \times\left[\alpha\left(1+t_{i s}\right)+(1-\alpha)\left(1-\left(1-t_{i s}\right) v_{r}\right) x_{\overparen{i r s}}\right]^{-1} \\
\times & \left\{\alpha+(1-\alpha) x_{\overparen{i r s}}\left[\left(1-\tau_{r s}\right) v_{r}+\tau_{r s}\left(1-v_{r}\right)\right.\right. \\
& \left.\left.\quad-2 \sqrt{\tau_{r s}\left(1-\tau_{r s}\right) v_{r}\left(1-v_{r}\right)}\left(1-2 w_{s}\right)\right]\right\}^{-1},
\end{aligned}
$$

as an angular integral,

$$
I_{v_{r}, w_{s}}=2^{1+4 \epsilon}\left(1+t_{i s}\right)^{-1}\left(2 \alpha+(1-\alpha) x_{\widetilde{i r s}}\right)^{-2} \frac{1}{\Omega_{d-3}} \int \mathrm{d} \Omega_{d-1}(q) \frac{1}{\left(p_{1} \cdot q\right)\left(p_{2} \cdot q\right)},
$$

where in a suitable frame,

$$
\begin{aligned}
& p_{1}^{\mu}=\left(1, \ldots,-\frac{\left(1-t_{i s}\right)(1-\alpha) x_{\widetilde{i r s}}}{\left(1+t_{i s}\right)\left(2 \alpha+(1-\alpha) x_{\overparen{i r s}}\right)}\right), \\
& p_{2}^{\mu}=\left(1, \ldots, \frac{(1-\alpha) x_{\overparen{i r s}}}{2 \alpha+(1-\alpha) x_{\overparen{i r s}}} \sin \chi, \frac{(1-\alpha) x_{\widetilde{i r s}}}{2 \alpha+(1-\alpha) x_{\overparen{i r s}}} \cos \chi\right)
\end{aligned}
$$

(the ... denotes vanishing components) and

$$
q^{\mu}=\left(1, . .{ }^{\prime} \text { angles'.., } \sin \vartheta \sin \varphi, \sin \vartheta \cos \varphi, \cos \vartheta\right) .
$$

(Here ..'angles'.. represents those angular variables that are trivial to integrate, since the integrand does not depend on them.) Clearly both $p_{1}$ and $p_{2}$ are massive and time-like, $p_{1}^{2}$, 
$p_{2}^{2}>0$, and $p_{1} \cdot p_{2}>0$. The angular integral can be written in terms of a Mellin-Barnes representation according to eq. (60) of ref. [57]. Finally, we can perform the Mellin-Barnes integrations at the expense of reintroducing two real integrations over the unit interval. We obtain a new two-dimensional real integral representation,

$$
\begin{aligned}
I_{v_{r}, w_{s}}= & -2^{1+4 \epsilon} \pi \epsilon\left(2 \alpha+(1-\alpha) x_{\widetilde{i r s}}\right)^{2-2 \epsilon} \\
\times & \int_{0}^{1} \mathrm{~d} s \mathrm{~d} u s^{-2 \epsilon} u^{-2 \epsilon}\left[2\left(1+t_{i s}\right) \alpha\left(\alpha+(1-\alpha) x_{\overparen{i r s}}\right)(1+u)\right. \\
& \left.+(1-\alpha)^{2}\left(1-\tau_{r s}+\tau_{r s} u\right) x_{\overparen{i r s}}^{2}+t_{i s}(1-\alpha)^{2}\left(\tau_{r s}+u-\tau_{r s} u\right) x_{\overparen{i r s}}^{2}\right]^{1-2 \epsilon} \\
\times & \left\{\left[\left(1+t_{i s}\right)^{2} \alpha\left(\alpha+(1-\alpha) x_{\overparen{i r s}}\right)+t_{i s}(1-\alpha)^{2} x_{\overparen{i r s}}^{2}\right]\left(2 \alpha+(1-\alpha) x_{\widetilde{i r s}}\right)^{4}(1-s)^{2} u^{2}\right. \\
& +\left[\alpha\left(\alpha+(1-\alpha) x_{\overparen{i r s}}\right) s(1-u)^{2}+\left(2 \alpha+(1-\alpha) x_{\widetilde{i r s}}\right)^{2} u\right] \\
\times & s\left[2\left(1+t_{i s}\right) \alpha\left(\alpha+(1-\alpha) x_{\overparen{i r s}}\right)(1+u)\right. \\
& \left.\left.+(1-\alpha)^{2}\left(1-\tau_{r s}+\tau_{r s} u\right) x_{\overparen{i r s}}^{2}+t_{i s}(1-\alpha)^{2}\left(\tau_{r s}+u-\tau_{r s} u\right) x_{\overparen{i r s}}^{2}\right]^{2}\right\}^{-1+\epsilon}
\end{aligned}
$$

free of square roots and singularities inside the integration region.

\section{Integrating the double collinear counterterm}

\section{D.1 Master integrals}

The double collinear momentum mapping leads to the exact factorisation of phase space as given by eq. (2.13), where the two-particle factorised phase space can be written as,

$$
\begin{aligned}
{\left[\mathrm{d} p_{2 ; m}^{(i r ; j s)}\left(p_{r}, p_{s}, \tilde{p}_{i r}, \tilde{p}_{j s} ; Q\right)\right]=} & \mathrm{d} \alpha \mathrm{d} \beta(1-\alpha-\beta)^{2(m-1)(1-\epsilon)} \Theta(\alpha) \Theta(\beta) \Theta(1-\alpha-\beta) \\
& \times \frac{s \widetilde{i r} Q}{2 \pi} \mathrm{d} \phi_{2}\left(p_{i}, p_{r} ; p_{(i r)}\right) \frac{s \widetilde{j s} Q}{2 \pi} \mathrm{d} \phi_{2}\left(p_{j}, p_{s} ; p_{(j s)}\right),
\end{aligned}
$$

where $p_{(i r)}^{\mu}=(1-\alpha-\beta) \tilde{p}_{i r}^{\mu}+\alpha Q^{\mu}$ and $p_{(j s)}^{\mu}=(1-\alpha-\beta) \tilde{p}_{j s}^{\mu}+\beta Q^{\mu}$.

When writing eq. (4.5), we have used that spin correlations generally present at the level of factorisation formulae cancel after azimuthal integration, since $k_{\perp, i, r}^{\mu}$ and $k_{\perp, j, s}^{\mu}$ as defined in ref. [22] are orthogonal to $\tilde{p}_{i r}^{\mu}$ and $\tilde{p}_{j s}^{\mu}$ respecitvely. Examining the actual form of the spin-averaged Altarelli-Parisi functions, and using the symmetry of the phase-space measure under the momentum exchanges $p_{i} \leftrightarrow p_{r}$, or $p_{j} \leftrightarrow p_{s}$, and under exchange of the pairs $\left(p_{i}, p_{r}\right) \leftrightarrow\left(p_{j}, p_{s}\right)$, we see that we must compute the following integrals,

$$
\begin{aligned}
\mathcal{I}_{2 \mathcal{C}, 6}^{(k, l)}\left(x_{\widetilde{i r}}, x_{\widetilde{j s}} ; \epsilon, \alpha_{0}, d_{0}\right)= & \left(\frac{(4 \pi)^{2}}{S_{\epsilon}} Q^{2 \epsilon}\right)^{2} \int_{2}\left[\mathrm{~d} p_{2 ; m}^{(i r ; j s)}\left(p_{r}, p_{s}, \tilde{p}_{i r}, \tilde{p}_{j s} ; Q\right)\right] \frac{1}{s_{i r}} \frac{1}{s_{j s}} \\
& \times z_{r, i}^{k} z_{s, j}^{l} f\left(\alpha_{0}, \alpha+\beta, d(m, \epsilon)\right),
\end{aligned}
$$

where $k, l=-1,0,1$ and 2 . By symmetry, we need only consider the cases when e.g., $k \leq l$. 


\section{D.2 Explicit representation}

To write the integral in eq. (D.2) explicitly, we must choose a specific representation of the factorised phase-space measure. We choose the scaled two-particle invariants $y_{i r}$ and $y_{j s}$ and rescaled momentum fractions $v_{r, i}$ and $v_{s, j}$,

$$
v_{r, i}=\frac{z_{r, i}-z_{r}^{(-)}}{z_{r}^{(+)}-z_{r}^{(-)}}, \quad v_{s, j}=\frac{z_{s, j}-z_{s}^{(-)}}{z_{s}^{(+)}-z_{s}^{(-)}}
$$

as integration variables. Here

$$
z_{r}^{(+)}=\frac{\alpha+(1-\alpha-\beta) x_{\widetilde{i r}}}{2 \alpha+(1-\alpha-\beta) x_{\widetilde{i r}}}, \quad z_{r}^{(-)}=\frac{\alpha}{2 \alpha+(1-\alpha-\beta) x_{\widetilde{i r}}},
$$

with similar expressions for $z_{s}^{(+)}$and $z_{s}^{(-)}$with $\alpha \rightarrow \beta$ and $i r \rightarrow j s$. In terms of $\alpha, \beta, v_{r, i}$ and $v_{s, j}$ we have

$$
\begin{aligned}
y_{i r} & =\alpha\left(\alpha+(1-\alpha-\beta) x_{\widetilde{i r}}\right), & y_{j s} & =\beta\left(\alpha+(1-\alpha-\beta) x_{\widetilde{j s}}\right), \\
z_{r, i} & =\frac{\alpha+(1-\alpha-\beta) x_{\widetilde{i r}} v_{r, i}}{2 \alpha+(1-\alpha-\beta) x_{\widetilde{i r}}}, & z_{s, j} & =\frac{\beta+(1-\alpha-\beta) x_{\widetilde{j s}} v_{s, j}}{2 \beta+(1-\alpha-\beta) x_{\widetilde{j s}}},
\end{aligned}
$$

and for the factorised phase-space measure we obtain

$$
\begin{aligned}
& {\left[\mathrm{d} p_{2 ; m}^{(i r ; j s)}\left(p_{r}, p_{s}, \tilde{p}_{i r}, \tilde{p}_{j s} ; Q\right)\right]=\left(\frac{S_{\epsilon}}{(4 \pi)^{2}} Q^{-2 \epsilon}\right)^{2}\left(Q^{2}\right)^{2} x_{\widetilde{i r} Q} x_{\widetilde{j s} Q}} \\
& \quad \times \mathrm{d} \alpha \mathrm{d} \beta(1-\alpha-\beta)^{2(m-1)(1-\epsilon)} \Theta(\alpha) \Theta(\beta) \Theta(1-\alpha-\beta) \\
& \quad \times \mathrm{d} y_{i r} \mathrm{~d} v_{r, i}\left[y_{i r} v_{r, i}\left(1-v_{r, i}\right)\right]^{-\epsilon} \delta\left(\alpha\left(\alpha+(1-\alpha-\beta) x_{\widetilde{i r}}\right)-y_{i r}\right) \\
& \quad \times \mathrm{d} y_{j s} \mathrm{~d} v_{s, j}\left[y_{j s} v_{s, j}\left(1-v_{s, j}\right)\right]^{-\epsilon} \delta\left(\beta\left(\beta+(1-\alpha-\beta) x_{\widetilde{j s}}\right)-y_{j s}\right) \\
& \quad \times \Theta\left(v_{r, i}\right) \Theta\left(1-v_{r, i}\right) \Theta\left(v_{s, j}\right) \Theta\left(1-v_{s, j}\right) .
\end{aligned}
$$

Using the parametrisation of eq. (D.7) and the expressions in eqs. (D.5) and (D.6), we find the following parametric integral representation for the master integral,

$$
\begin{aligned}
& \mathcal{I}_{2 \mathcal{C}, 6}^{(k, l)}\left(x_{\widetilde{i r}}, x_{\widetilde{j s}} ; \epsilon, \alpha_{0}, d_{0}\right)=x_{\widetilde{i r}} x_{\widetilde{j s}} \int_{0}^{1} \mathrm{~d} \alpha \mathrm{d} \beta \Theta\left(\alpha_{0}-\alpha-\beta\right) \int_{0}^{1} \mathrm{~d} v \mathrm{~d} u \\
& \quad \times \alpha^{-1-\epsilon} \beta^{-1-\epsilon}(1-\alpha-\beta)^{2 d_{0}-2(1-\epsilon)} v^{-\epsilon}(1-v)^{-\epsilon} u^{-\epsilon}(1-u)^{-\epsilon} \\
& \quad \times\left[\alpha+(1-\alpha-\beta) x_{\widetilde{i r}}\right]^{-1-\epsilon}\left[\beta+(1-\alpha-\beta) x_{\widetilde{j s}}\right]^{-1-\epsilon} \\
& \quad \times\left(\frac{\alpha+(1-\alpha-\beta) x_{\widetilde{i r}} v}{2 \alpha+(1-\alpha-\beta) x_{\widetilde{i r}}}\right)^{k}\left(\frac{\beta+(1-\alpha-\beta) x_{\widetilde{j s}} u}{2 \beta+(1-\alpha-\beta) x_{\widetilde{j s}}}\right)^{l} .
\end{aligned}
$$

Eq. (D.8) is directly suitable for treatment with general purpose sector decomposition codes. 


\section{E Integrating the soft collinear counterterms}

\section{E.1 Master integrals}

The consecutive collinear and soft mappings lead to the exact factorisation of phase space as given by eq. (2.14), where the one-particle factorised phase spaces can be written in the following form. For the collinear mapping we have

$$
\left[\mathrm{d} p_{1 ; m+1}^{(i r)}\left(p_{r}, \hat{p}_{i r} ; Q\right)\right]=\mathrm{d} \alpha(1-\alpha)^{2 m(1-\epsilon)-1} \frac{s_{\text {ir } Q}}{2 \pi} \mathrm{d} \phi_{2}\left(p_{i}, p_{r} ; p_{(i r)}\right) \Theta(\alpha) \Theta(1-\alpha),
$$

where $p_{(i r)}^{\mu}=(1-\alpha) \hat{p}_{i r}^{\mu}+\alpha Q^{\mu}$. For the soft mapping we find

$$
\left[\mathrm{d} p_{1 ; m}^{(\hat{s})}\left(\hat{p}_{s}, K ; Q\right)\right]=\mathrm{d} y(1-y)^{(m-1)(1-\epsilon)} \frac{Q^{2}}{2 \pi} \mathrm{d} \phi_{2}\left(\hat{p}_{s}, K ; Q\right) \Theta(y) \Theta(1-y),
$$

where $y \equiv y_{\hat{s} Q}$ and the momentum $K$ is massive with $K^{2}=(1-y) Q^{2}$. As the notation above indicates, $\alpha$ and $y$ are integration variables.

To integrate eqs. (4.8)-(4.13) over the factorised phase spaces of eqs. (E.1) and (E.2), we can pass to the azimuthally averaged Altarelli-Parisi splitting functions, since the azimuthal correlations generally present in eqs. (4.8)-(4.13) vanish after integration by the usual argument (the transverse momentum $\tilde{k}_{\perp, i, r}^{\mu}$ in the splitting kernels is defined to be orthogonal to the parent momentum $\tilde{p}_{i r}^{\mu}$ ). The Altarelli-Parisi functions can be expressed as linear combinations of powers of momentum fractions, so we need to compute integrals of the form,

$$
\begin{aligned}
\left(\frac{(4 \pi)^{2}}{S_{\epsilon}} Q^{2 \epsilon}\right)^{2} & \int_{2}\left[\mathrm{~d} p_{1 ; m+1}^{(i r)}\left(p_{r}, \hat{p}_{i r} ; Q\right)\right]\left[\mathrm{d} p_{1 ; m}^{(\hat{s})}\left(\hat{p}_{s} ; Q\right)\right]\left\{\frac{1}{2} \mathcal{S}_{j k}(s), \frac{2}{s_{(i r) s}} \frac{1-z_{s, i r}}{z_{s, i r}}, \frac{2}{s_{j s}} \frac{z_{j, s}}{z_{s, j}}\right\} \\
& \times \frac{1}{s_{i r}} z_{r, i}^{l} f\left(\alpha_{0}, \alpha, d(m, \epsilon)\right) f\left(y_{0}, y, d(m, \epsilon)\right) .
\end{aligned}
$$

We perform the integration over $\left[\mathrm{d} p_{1 ; m+1}^{(i r)}\right]$ first, so we need to write the integrands in terms of $\hat{p}_{s}^{\mu}$ and tilded momenta. The singly collinear mapping of the momenta rescales the invariants which do not involve the collinear pair,

$$
y_{j k}=(1-\alpha)^{2} y_{\hat{j} \hat{k}} \quad \text { and } \quad y_{j Q}=(1-\alpha) y_{\hat{j} Q},
$$

while those which involve the collinear momenta become

$$
y_{(i r) k}=(1-\alpha)\left[(1-\alpha) y_{\widehat{i r} \hat{k}}+\alpha y_{\hat{k} Q}\right] \quad \text { and } \quad y_{(i r) Q}=(1-\alpha) y_{\widehat{i r} Q}+2 \alpha .
$$

The successive singly soft mapping of the momenta rescales those two-particle invariants that do not involve the soft parton,

$$
y_{\hat{j} \hat{k}}=(1-y) y_{\tilde{j} \tilde{k}},
$$

while those involving the soft parton $s$ become $y_{\hat{j} \hat{s}}=y_{\tilde{j} \hat{s}}$. Finally, we have

$$
y_{\hat{j} Q}=(1-y) y_{\tilde{j} Q}+y_{\tilde{j} \hat{s}},
$$


for $j \neq s$, while $y_{\hat{s} Q}=y$ is simply an integration variable. Therefore, the integrands are expressed as follows. For the eikonal factor, we find

$$
\mathcal{S}_{j k}(s)=\frac{1-y}{(1-\alpha)^{2}} \mathcal{S}_{\widetilde{j} \widetilde{k}}(\hat{s}),
$$

if $j$ and $k$ are distinct from $(i r)$, while

$$
\frac{1}{2} \mathcal{S}_{(i r) k}(s)=\frac{1-y}{(1-\alpha)^{2}} \frac{(1-\alpha) s_{\widetilde{i r} \tilde{k}}+\alpha s_{\tilde{k} Q}}{\left[(1-\alpha) s_{\widetilde{i r} \hat{s}}+\alpha s_{\hat{s} Q}\right] s_{\tilde{k} \hat{s}}}+\frac{\alpha}{(1-\alpha)^{2}} \frac{s_{\tilde{k} \hat{s}}}{\left[(1-\alpha) s_{\widetilde{i r} \hat{s}}+\alpha s_{\hat{s} Q}\right] s_{\tilde{k} \hat{s}}},
$$

if e.g., $j$ coincides with $(i r)$. Finally, we have

$$
\frac{2}{s_{(i r) s}} \frac{1-z_{s, i r}}{z_{s, i r}}=\frac{2}{s_{(i r) s}} \frac{s_{(i r) Q}}{s_{s Q}}=\frac{2}{(1-\alpha)^{2}} \frac{2 \alpha+(1-\alpha)\left[(1-y) s_{\widetilde{i r} Q}+s_{\widetilde{i r} \hat{s}}\right]}{\left[(1-\alpha) s_{\widetilde{i r} \hat{s}}+\alpha s_{\hat{s} Q}\right] s_{\hat{s} Q}}
$$

and

$$
\frac{2}{s_{j s}} \frac{z_{j, s}}{z_{s, j}}=\frac{2}{s_{j s}} \frac{s_{j Q}}{s_{s} Q}=\frac{2}{(1-\alpha)^{2} s_{\tilde{j} \hat{s}}} \frac{(1-y) s_{\tilde{j} Q}+s_{\tilde{j} \hat{s}}}{s_{\hat{s} Q}} .
$$

Hence, we define five soft collinear master integrals,

$$
\begin{aligned}
& \mathcal{I}_{2 \mathcal{C S}, 1}^{(l)}\left(x_{\widetilde{i r}}, Y_{\widetilde{i r} \widetilde{j}, Q}, Y_{\widetilde{i r} \widetilde{k}, Q}, Y_{\widetilde{j} \widetilde{k}, Q} ; \epsilon, \alpha_{0}, y_{0}, d_{0}, d_{0}^{\prime}\right)= \\
&=-\left(\frac{(4 \pi)^{2}}{S_{\epsilon}} Q^{2 \epsilon}\right)^{2} \int_{2}\left[\mathrm{~d} p_{1 ; m+1}^{(i r)}\left(p_{r}, \hat{p}_{i r} ; Q\right)\right]\left[\mathrm{d} p_{1 ; m}^{(\hat{s})}\left(\hat{p}_{s} ; Q\right)\right] \\
& \times \frac{1}{2} \frac{1-y}{(1-\alpha)^{2}} \mathcal{S}_{\widetilde{j} \widetilde{k}}(\hat{s}) \frac{1}{s_{i r}} z_{r, i}^{l} \\
& \times f\left(\alpha_{0}, \alpha, d(m, \epsilon)\right) f\left(y_{0}, y, d(m, \epsilon)\right), \\
& \mathcal{I}_{2 \mathcal{C S}, 2}^{(l)}\left(x_{\widetilde{i r}}, Y_{\widetilde{i r} \widetilde{k}, Q} ; \epsilon, \alpha_{0}, y_{0}, d_{0}, d_{0}^{\prime}\right)= \\
&=-\left(\frac{(4 \pi)^{2}}{S_{\epsilon}} Q^{2 \epsilon}\right)^{2} \int_{2}\left[\mathrm{~d} p_{1 ; m+1}^{(i r)}\left(p_{r}, \hat{p}_{i r} ; Q\right)\right]\left[\mathrm{d} p_{1 ; m}^{(\hat{s})}\left(\hat{p}_{s} ; Q\right)\right] \\
& \quad \times \frac{1-y}{(1-\alpha)^{2}} \frac{(1-\alpha) s_{\widetilde{i r} \tilde{k}}+\alpha s_{\tilde{k} Q}}{\left[(1-\alpha) s_{\widetilde{i r} \hat{s}}+\alpha s_{\hat{s} Q}\right] s_{\tilde{k} \hat{s}}} \frac{1}{s_{i r}} z_{r, i}^{l} \\
& \quad \times f\left(\alpha_{0}, \alpha, d(m, \epsilon)\right) f\left(y_{0}, y, d(m, \epsilon)\right), \\
& \mathcal{I}_{2 \mathcal{C S}, 3}^{(l)}\left(x_{\widetilde{i r}} ; \epsilon, \alpha_{0}, y_{0}, d_{0}, d_{0}^{\prime}\right)= \\
&=-\left(\frac{(4 \pi)^{2}}{S_{\epsilon}} Q^{2 \epsilon}\right)^{2} \int_{2}\left[\mathrm{~d} p_{1 ; m+1}^{(i r)}\left(p_{r}, \hat{p}_{i r} ; Q\right)\right]\left[\mathrm{d} p_{1 ; m}^{(\hat{s})}\left(\hat{p}_{s} ; Q\right)\right] \\
& \quad \times \frac{\alpha}{(1-\alpha)^{2}} \frac{1}{\left((1-\alpha) s_{\widetilde{i r} \hat{s}}+\alpha s_{\hat{s} Q}\right] s_{\tilde{k} \hat{s}}} \frac{1}{s_{i r}} z_{r, i}^{l} \\
& \quad \times f\left(\alpha_{0}, \alpha, d(m, \epsilon)\right) f\left(y_{0}, y, d(m, \epsilon)\right),
\end{aligned}
$$




$$
\begin{aligned}
\mathcal{I}_{2 \mathcal{L S}, 4}^{(l)}\left(x_{\widetilde{i r}} ; \epsilon, \alpha_{0}, y_{0}, d_{0}, d_{0}^{\prime}\right)= \\
=\left(\frac{(4 \pi)^{2}}{S_{\epsilon}} Q^{2 \epsilon}\right)^{2} \int_{2}\left[\mathrm{~d} p_{1 ; m+1}^{(i r)}\left(p_{r}, \hat{p}_{i r} ; Q\right)\right]\left[\mathrm{d} p_{1 ; m}^{(\hat{s})}\left(\hat{p}_{s} ; Q\right)\right] \\
\quad \times \frac{2}{(1-\alpha)^{2}} \frac{2 \alpha+(1-\alpha)\left[(1-y) s_{\widetilde{i r} Q}+s_{\widetilde{i r} \hat{s}}\right]}{s_{i r}} \frac{1}{\left[(1-\alpha) s_{\widetilde{i r} \hat{s}}+\alpha s_{\hat{s} Q}\right] s_{\hat{s} Q}^{l}} \\
\quad \times f\left(\alpha_{0}, \alpha, d(m, \epsilon)\right) f\left(y_{0}, y, d(m, \epsilon)\right)
\end{aligned}
$$

and

$$
\begin{aligned}
& \mathcal{I}_{2 \mathcal{C S}, 5}^{(l)}\left(x_{\widetilde{i r}}, Y_{\widetilde{i r} \tilde{j}, Q} ; \epsilon, \alpha_{0}, y_{0}, d_{0}, d_{0}^{\prime}\right)= \\
&=\left(\frac{(4 \pi)^{2}}{S_{\epsilon}} Q^{2 \epsilon}\right)^{2} \int_{2}\left[\mathrm{~d} p_{1 ; m+1}^{(i r)}\left(p_{r}, \hat{p}_{i r} ; Q\right)\right]\left[\mathrm{d} p_{1 ; m}^{(\hat{s})}\left(\hat{p}_{s} ; Q\right)\right] \\
& \quad \times \frac{2}{(1-\alpha)^{2} s_{\tilde{j} \hat{s}}} \frac{(1-y) s_{\tilde{j} Q}+s_{\tilde{j} \hat{s}}}{s_{\hat{s} Q}} \frac{1}{s_{i r}} z_{r, i}^{l} \\
& \quad \times f\left(\alpha_{0}, \alpha, d(m, \epsilon)\right) f\left(y_{0}, y, d(m, \epsilon)\right) .
\end{aligned}
$$

We need to compute these integrals for $l=-1,0,1,2$.

\section{E.2 Explicit representations}

Eqs. (E.12)-(E.16) are very similar to the iterated collinear — soft collinear integrals we computed in ref. [31], and we employ the techniques of ref. [31] for computing the integrals $\mathcal{I}_{2 \mathcal{C S}, n}^{(l)}$. We begin by recalling the specific representation of the factorised phase-space measures in eqs. (E.1) and (E.2). For the singly collinear measure in eq. (E.1), we choose the scaled two-particle invariant $y_{i r}$ and the rescaled momentum fraction $v_{r, i}$,

$$
v_{r, i}=\frac{z_{r, i}-z_{r}^{(-)}}{z_{r}^{(+)}-z_{r}^{(-)}},
$$

as integration variables. Here

$$
z_{r}^{(+)}=\frac{\alpha+(1-\alpha) x_{\widehat{i r}}}{2 \alpha+(1-\alpha) x_{\widehat{i r}}}, \quad z_{r}^{(-)}=\frac{\alpha}{2 \alpha+(1-\alpha) x_{\widehat{i r}}} .
$$

In terms of $\alpha$ and $v_{r, i}$, we have

$$
y_{i r}=\alpha\left(\alpha+(1-\alpha) x_{\widehat{i r}}\right), \quad \text { and } \quad z_{r, i}=\frac{\alpha+(1-\alpha) x_{\widehat{i r}} v_{r, i}}{2 \alpha+(1-\alpha) x_{\widehat{i r}}}
$$

while the phase-space measure $\left[\mathrm{d} p_{1 ; m+1}^{(i r)}\left(p_{r}, \hat{p}_{i r} ; Q\right)\right]$ reads

$$
\begin{aligned}
{\left[\mathrm{d} p_{1 ; m+1}^{(i r)}\left(p_{r}, \hat{p}_{i r} ; Q\right)\right]=} & \frac{S_{\epsilon}}{(4 \pi)^{2}}\left(Q^{2}\right)^{1-\epsilon} x_{\widehat{i r}} \mathrm{~d} \alpha \mathrm{d} y_{i r} \mathrm{~d} v_{r, i}(1-\alpha)^{2 m(1-\epsilon)-1}\left[y_{i r} v_{r, i}\left(1-v_{r, i}\right)\right]^{-\epsilon} \\
& \times \delta\left(\alpha\left(\alpha+(1-\alpha) x_{\widehat{i r}}\right)-y_{i r}\right) \Theta(\alpha) \Theta(1-\alpha) \Theta\left(v_{r, i}\right) \Theta\left(1-v_{r, i}\right) .
\end{aligned}
$$


Turning to $\left[\mathrm{d} p_{1: m}^{(\hat{s})}\left(\hat{p}_{s}, K ; Q\right)\right]$ in eq. (E.2), we use the (scaled) energy and the angular variables of $\hat{p}_{s}^{\mu}$ in some specific Lorentz frame to write the two-particle phase space $\mathrm{d} \phi_{2}\left(\hat{p}_{s}, K ; Q\right)$ explicitly. In particular, we work in the rest frame of $Q^{\mu}$, where $Q^{\mu}=$ $\sqrt{s}(1, \ldots)$ and $\hat{p}_{s}^{\mu}$ is parametrised as,

$$
\hat{p}_{s}^{\mu}=\hat{E}_{s}\left(1, . .{ }^{\prime} \text { angles'.., } \sin \vartheta \sin \varphi \sin \eta, \sin \vartheta \sin \varphi \cos \eta, \sin \vartheta \cos \varphi, \cos \vartheta\right) .
$$

The specific orientation of this frame is chosen below according to what is most convenient for computing each integral. However, independent of orientation, in terms of the scaled energy-like variable,

$$
\varepsilon_{\hat{s}}=\frac{2 \hat{p}_{s} \cdot Q}{Q^{2}}=\frac{2 \hat{E}_{s}}{\sqrt{s}}
$$

and the angular variables $\vartheta, \varphi$ and $\eta$, the two-particle phase space $\mathrm{d} \phi_{2}\left(\hat{p}_{s}, K ; Q\right)$ reads

$$
\begin{aligned}
\mathrm{d} \phi_{2}\left(\hat{p}_{s}, K ; Q\right)= & \frac{\left(Q^{2}\right)^{-\epsilon}}{(4 \pi)^{2}} S_{\epsilon}\left(-2^{2 \epsilon} \epsilon\right) \mathrm{d} \varepsilon_{\hat{s}} \varepsilon_{\hat{s}}^{1-2 \epsilon} \delta\left(y-\varepsilon_{\hat{s}}\right) \\
& \times \mathrm{d}(\cos \vartheta) \mathrm{d}(\cos \varphi) \mathrm{d}(\cos \eta)(\sin \vartheta)^{-2 \epsilon}(\sin \varphi)^{-1-2 \epsilon}(\sin \eta)^{-2-2 \epsilon}
\end{aligned}
$$

We are now ready to display the master integrals.

Integrated soft collinear counterterm for $i, j, k$ distinct. This case leads to the integral $\mathcal{I}_{2 \mathcal{C S}, 1}$. We choose the orientation of the frame such that the momenta appearing in the integrand take the following forms,

$$
\begin{aligned}
& \tilde{p}_{j}^{\mu}=\tilde{E}_{j}(1, \ldots, 1), \quad \tilde{p}_{k}^{\mu}=\tilde{E}_{k}\left(1, \ldots, \sin \chi_{k}, \cos \chi_{k}\right) \\
& \tilde{p}_{i r}^{\mu}=\tilde{E}_{i r}\left(1, \ldots, \sin \phi_{i r} \sin \chi_{i r}, \cos \phi_{i r} \sin \chi_{i r}, \cos \chi_{i r}\right) .
\end{aligned}
$$

Then, expressing the various invariants in terms of the integration variables in the chosen frame of eq. (E.24), we find

$$
\begin{aligned}
y_{\tilde{j} \hat{s}} & =\frac{1}{2} y_{\tilde{j} Q} y(1-\cos \vartheta), \\
y_{\tilde{k} \hat{s}} & =\frac{1}{2} y_{\tilde{k} Q} y\left(1-\sin \chi_{k} \sin \vartheta \cos \varphi-\cos \chi_{k} \cos \vartheta\right), \\
y_{\widetilde{i r} \hat{s}} & =\frac{1}{2} x_{\tilde{i r}} y\left(1-\sin \phi_{i r} \sin \chi_{i r} \sin \vartheta \sin \varphi \cos \eta-\cos \phi_{i r} \sin \chi_{i r} \sin \vartheta \cos \varphi-\cos \chi_{i r} \cos \vartheta\right),
\end{aligned}
$$

where

$$
\begin{aligned}
\cos \chi_{k} & =\cos \chi\left(Y_{\widetilde{j} \widetilde{k}, Q}\right), \quad \cos \chi_{i r}=\cos \chi\left(Y_{\widetilde{j} \widetilde{i r}, Q}\right) \\
\cos \phi_{i r} & =\frac{Y_{\widetilde{j} \widetilde{k}, Q}+Y_{\widetilde{j} \widetilde{i r}, Q}-Y_{\widetilde{k} \widetilde{i r}, Q}-2 Y_{\widetilde{j} \widetilde{k}, Q} Y_{\widetilde{j} \widetilde{i r}, Q}}{\sin \chi\left(Y_{\widetilde{j} \widetilde{k}, Q}\right) \sin \chi\left(Y_{\widetilde{j} \widetilde{i r}, Q}\right)},
\end{aligned}
$$

with

$$
\cos \chi(Y)=1-2 Y, \quad \sin \chi(Y)=2 \sqrt{Y(1-Y)}
$$


The factor $y_{\hat{k} \hat{s}}$ in the denominator of the eikonal factor vanishes at $\cos \varphi=1$ and $\cos \vartheta=\cos \chi_{k}$. Hence, the integrand has a line singularity that we remove by performing partial fractioning as in refs. [31, 58],

$$
\frac{s_{\tilde{j} \tilde{k}}}{s_{\tilde{j} \hat{s}} s_{\tilde{k} \hat{s}}}=\frac{1}{Q^{2}} 4 Y_{\tilde{j} \tilde{k}, Q}\left(\frac{y_{\tilde{j} Q}}{2 y_{\tilde{j} \hat{s}}}+\frac{y_{\tilde{k} Q}}{2 y_{\tilde{k} \hat{s}}}\right)\left(\frac{2 y_{\tilde{j} \hat{s}}}{y_{\tilde{j} Q}}+\frac{2 y_{\tilde{k} \hat{s}}}{y_{\tilde{k} Q}}\right)^{-1} \text {. }
$$

Then we find

$$
\begin{aligned}
\mathcal{I}_{2 \mathcal{C S}, 1}^{(l)} & \left(x_{\widetilde{i r}}, Y_{\widetilde{i r} \widetilde{j}, Q}, Y_{\widetilde{i r} \widetilde{k}, Q}, Y_{\widetilde{j} \widetilde{k}, Q} ; \epsilon, \alpha_{0}, y_{0}, d_{0}, d_{0}^{\prime}\right)=-\left(\frac{-2^{2 \epsilon} \epsilon}{2 \pi}\right) 4 Y_{\widetilde{j} \widetilde{k}, Q} \\
& \times \int_{0}^{y_{0}} \mathrm{~d} y y^{1-2 \epsilon}(1-y)^{d_{0}^{\prime}-1+\epsilon} \\
& \times \int_{-1}^{1} \mathrm{~d}(\cos \vartheta) \mathrm{d}(\cos \varphi) \mathrm{d}(\cos \eta)(\sin \vartheta)^{-2 \epsilon}(\sin \varphi)^{-1-2 \epsilon}(\sin \eta)^{-2-2 \epsilon} \\
& \times \int_{0}^{\alpha_{0}} \mathrm{~d} \alpha \alpha^{-1-\epsilon}(1-\alpha)^{2 d_{0}-3}\left\{\alpha+(1-\alpha)\left[(1-y) x_{\widetilde{i r}}+y_{\widetilde{i r} \hat{s}}\right]\right\}^{-1-\epsilon} \\
& \times \int_{0}^{1} \mathrm{~d} v v^{-\epsilon}(1-v)^{-\epsilon}\left(\frac{\alpha+(1-\alpha)\left[(1-y) x_{\widetilde{i r}}+y_{\widetilde{i r} \hat{s} \hat{s}}\right] v}{2 \alpha+(1-\alpha)\left[(1-y) x_{\widetilde{i r}}+y_{\widetilde{i r} \hat{s}}\right]}\right)^{l} \\
& \times\left[(1-y) x_{\widetilde{i r}}+y_{\widetilde{i r} \hat{s}}\right]\left(\frac{y_{\tilde{j} Q}}{2 y_{\tilde{j} \hat{s}}}+\frac{y_{\tilde{k} Q}}{2 y_{\tilde{k} \hat{s}}}\right)\left(\frac{2 y_{\tilde{j} \hat{s}}}{y_{\tilde{j} Q}}+\frac{2 y_{\tilde{k} \hat{s}}}{y_{\tilde{k} Q}}\right)^{-1},
\end{aligned}
$$

where $y_{\tilde{j} \hat{s}}, y_{\tilde{k} \hat{s}}$ and $y_{\widetilde{i r} \hat{s}}$ are understood to be functions of the integration variables as given in eqs. (E.25)-(E.27). We also used $y_{\widetilde{\text { ir }} Q}=x_{\widetilde{\text { ir }}}$, and performed the integration over $\varepsilon_{\hat{s}}$ with the $\delta$-function in eq. (E.23). The choice of frame in eq. (E.24) is convenient for integrating the first term in the partial fraction, while the second term is more straightforward to integrate in a frame where $k$ and $j$ are exchanged. Upon performing this exchange, we find that the functional form of the master integral is unchanged, and we must simply interchange $Y_{\widetilde{j} \widetilde{i r}, Q}$ with $Y_{\widetilde{k} \widetilde{i r}, Q}$ to obtain one term from the other.

The integral $\mathcal{I}_{2 \mathcal{C S}, 1}^{(l)}$ as given in its most general form in eq. (E.31) first appears in electron-positron annihilation into four or more jets at NNLO. For a three-jet computation, the $\eta$ integral is trivial, since momentum conservation forces the three final state momenta to be coplanar, and hence we find $\phi_{i r}=0$ or $\pi$ in eq. (E.24). We display the resulting simplifications. Choosing $\phi_{i r}=\pi$, eq. (E.24) becomes

$$
\begin{aligned}
& \tilde{p}_{j}^{\mu}=\tilde{E}_{j}(1, \ldots, 1), \quad \tilde{p}_{k}^{\mu}=\tilde{E}_{k}\left(1, \ldots, \sin \chi_{k}, \cos \chi_{k}\right), \\
& \tilde{p}_{i r}^{\mu}=\tilde{E}_{i r}\left(1, \ldots,-\sin \chi_{i r}, \cos \chi_{i r}\right),
\end{aligned}
$$

which leads to

$$
\begin{aligned}
y_{\tilde{j} \hat{s}} & =\frac{1}{2} y_{\tilde{j} Q} y(1-\cos \vartheta), \\
y_{\tilde{k} \hat{s}} & =\frac{1}{2} y_{\tilde{k} Q} y\left(1-\sin \chi_{k} \sin \vartheta \cos \varphi-\cos \chi_{k} \cos \vartheta\right), \\
y_{\widetilde{i r} \hat{s}} & =\frac{1}{2} x_{\widetilde{i r}} y\left(1+\sin \chi_{i r} \sin \vartheta \cos \varphi-\cos \chi_{i r} \cos \vartheta\right),
\end{aligned}
$$


with $\cos \chi_{k}=\cos \chi\left(Y_{\widetilde{j} \widetilde{k}, Q}\right)$ and $\cos \chi_{i r}=\cos \chi\left(Y_{\widetilde{j} \widetilde{i r}, Q}\right)$ as in eq. (E.28). Then the integration over $\eta$ can be performed using

$$
\int_{-1}^{1} \mathrm{~d}(\cos \eta)(\sin \eta)^{-2-2 \epsilon}=-\frac{2^{-2 \epsilon}}{\epsilon} \frac{\Gamma^{2}(1-\epsilon)}{\Gamma(1-2 \epsilon)}
$$

Due to momentum conservation, only two out of the five kinematic variables in eqs. (E.33)-(E.35) are independent. E.g., in terms of $Y_{\widetilde{j} \widetilde{k}, Q}$ and $Y_{\widetilde{j} \widetilde{i r}, Q}$, we have

$$
\begin{aligned}
& y_{\tilde{j} Q} \equiv x_{\tilde{j}}=\frac{\left(2 Y_{\widetilde{j} \widetilde{k}, Q}-1\right) \sqrt{Y_{\widetilde{j} \widetilde{i r}, Q}\left(1-Y_{\widetilde{j} \widetilde{i r}, Q}\right)}+\left(2 Y_{\widetilde{j} \widetilde{i r}, Q}-1\right) \sqrt{Y_{\widetilde{j} \tilde{k}, Q}\left(1-Y_{\widetilde{j} \tilde{k}, Q}\right)}}{Y_{\widetilde{j} \widetilde{k}, Q} \sqrt{Y_{\widetilde{j} \widetilde{i r}, Q}\left(1-Y_{\widetilde{j} \widetilde{i r}, Q}\right)}+Y_{\widetilde{j} \widetilde{i r}, Q} \sqrt{Y_{\widetilde{j} \widetilde{k}, Q}\left(1-Y_{\widetilde{j} \widetilde{k}, Q}\right)}}, \\
& y_{\tilde{k} Q} \equiv x_{\tilde{k}}=\frac{\sqrt{Y_{\widetilde{j} \widetilde{i r}, Q}\left(1-Y_{\widetilde{j} \widetilde{i r}, Q}\right)}}{Y_{\widetilde{j} \widetilde{k}, Q} \sqrt{Y_{\widetilde{j} \widetilde{i r}, Q}\left(1-Y_{\widetilde{j} \widetilde{i r}, Q}\right)}+Y_{\widetilde{j} \widetilde{i r}, Q} \sqrt{Y_{\widetilde{j} \widetilde{k}, Q}\left(1-Y_{\widetilde{j} \widetilde{k}, Q}\right)}}, \\
& x_{\widetilde{i r}}=\frac{\sqrt{Y_{\widetilde{j} \widetilde{k}, Q}\left(1-Y_{\widetilde{j} \widetilde{k}, Q}\right)}}{Y_{\widetilde{j} \widetilde{k}, Q} \sqrt{Y_{\widetilde{j} \widetilde{i r}, Q}\left(1-Y_{\widetilde{j} \widetilde{i r}, Q}\right)}+Y_{\widetilde{j} \widetilde{i r}, Q} \sqrt{Y_{\widetilde{j} \widetilde{k}, Q}\left(1-Y_{\widetilde{j} \widetilde{k}, Q}\right)}} .
\end{aligned}
$$

Note that $Y_{\widetilde{k} \widetilde{i r}, Q}$ is also easily expressed with $Y_{\widetilde{j} \widetilde{k}, Q}$ and $Y_{\widetilde{j} \widetilde{i r}, Q}$,

$$
Y_{\widetilde{k} \widetilde{i r}, Q}=Y_{\widetilde{j} \widetilde{k}, Q}+Y_{\widetilde{j} \widetilde{i r}, Q}-2 Y_{\widetilde{j} \widetilde{k}, Q} Y_{\widetilde{j} \widetilde{i r}, Q}+2 \sqrt{Y_{\widetilde{j} \widetilde{k}, Q}\left(1-Y_{\widetilde{j} \widetilde{k}, Q}\right) Y_{\widetilde{j} \widetilde{i r}, Q}\left(1-Y_{\widetilde{j} \widetilde{i r}, Q}\right)} .
$$

The physical region in $Y_{\widetilde{j} \widetilde{k}, Q}$ and $Y_{\widetilde{j} \widetilde{i r}, Q}$ is simply given by $0<Y_{\widetilde{j} \widetilde{k}, Q}, Y_{\widetilde{j} \widetilde{i r}, Q}<1$ and $Y_{\widetilde{j} \widetilde{k}, Q}+Y_{\widetilde{j} \widetilde{i r}, Q}>1$.

In the case of two-jet production, this integral does not appear at all because we do not have three independent hard final state momenta.

Integrated soft collinear counterterm for $j=(i r)$. This case leads to the integrals $\mathcal{I}_{2 \mathcal{L S ~}, 2}^{(l)}$ and $\mathcal{I}_{2 \mathcal{C S}, 3}^{(l)}$. For $\mathcal{I}_{2 \mathcal{L S}, 2}^{(l)}$ the integrand again has a line singularity, which we can remove via partial fractioning. Using

$$
\begin{aligned}
& \frac{(1-\alpha) s_{\widetilde{i r} \tilde{k}}+\alpha s_{\tilde{k} Q}}{\left[(1-\alpha) s_{\widetilde{i r} \hat{s}}+\alpha s_{\hat{s} Q}\right] s_{\tilde{k} \hat{s}}}=\frac{1}{Q^{2}} 4 \frac{\alpha+(1-\alpha) x_{\widetilde{i r}} Y_{\widetilde{i r} \widetilde{k}, Q}}{y^{2}} \\
& \quad \times\left(\frac{1}{2 \alpha+(1-\alpha) x_{\widetilde{i r}} \frac{2 y_{\widetilde{i_{\tilde{i r}}} \hat{s}}}{y x_{\tilde{i r}}}}+\frac{y y_{\tilde{k} Q}}{2 y_{\tilde{k} \hat{s}}}\right)\left(2 \alpha+(1-\alpha) x_{\widetilde{i r}} \frac{2 y_{\widetilde{i r} \hat{s}}}{y x_{\widetilde{i r}}}+\frac{2 y_{\tilde{k} \hat{s}}}{y y_{\tilde{k} Q}}\right)^{-1},
\end{aligned}
$$


we find the following parametric integral representation,

$$
\begin{aligned}
& \mathcal{I}_{2 \mathcal{S}, 2}^{(l)}\left(x_{\widetilde{i r}}, Y_{\widetilde{i r} \widetilde{k}, Q} ; \epsilon, \alpha_{0}, y_{0}, d_{0}, d_{0}^{\prime}\right)=-\frac{\Gamma^{2}(1-\epsilon)}{2 \pi \Gamma(1-2 \epsilon)} 4 \int_{0}^{y_{0}} \mathrm{~d} y y^{-1-2 \epsilon}(1-y)^{d_{0}^{\prime}-1+\epsilon} \\
& \quad \times \int_{-1}^{1} \mathrm{~d}(\cos \vartheta) \mathrm{d}(\cos \varphi)(\sin \vartheta)^{-2 \epsilon}(\sin \varphi)^{-1-2 \epsilon}\left[(1-y) x_{\widetilde{i r}}+y_{\widetilde{i r} \hat{s}}\right] \\
& \quad \times \int_{0}^{\alpha_{0}} \mathrm{~d} \alpha \alpha^{-1-\epsilon}(1-\alpha)^{2 d_{0}-3}\left\{\alpha+(1-\alpha)\left[(1-y) x_{\widetilde{i r}}+y_{\widetilde{i r} \hat{s}}\right]\right\}^{-1-\epsilon} \\
& \quad \times \int_{0}^{1} \mathrm{~d} v v^{-\epsilon}(1-v)^{-\epsilon}\left[\alpha+(1-\alpha) x_{\widetilde{i r}} Y_{\widetilde{i r} \widetilde{k}, Q}\right]\left(\frac{1}{\left.2 \alpha+(1-\alpha) x_{\widetilde{i r}} \frac{2 y_{\widetilde{i r} \hat{s}}}{y x_{\tilde{i r}}}+\frac{y y_{\tilde{k} Q}}{2 y_{\tilde{k} \hat{s}}}\right)}\right. \\
& \quad \times\left(2 \alpha+(1-\alpha) x_{\widetilde{i r}} \frac{2 y_{\widetilde{i r} \hat{s}}}{y x_{\widetilde{i r}}}+\frac{2 y_{\tilde{k} \hat{s}}}{y y_{\tilde{k} Q}}\right)^{-1}\left(\frac{\alpha+(1-\alpha)\left[(1-y) x_{\widetilde{i r}}+y_{\widetilde{i r} \hat{s}}\right] v}{2 \alpha+(1-\alpha)\left[(1-y) x_{\widetilde{i r}}+y_{\widetilde{i r} \hat{s}}\right]}\right)^{l} .
\end{aligned}
$$

The two terms in the partial fraction are evaluated most conveniently in two different frames. In the first one

$$
\tilde{p}_{i r}^{\mu}=\tilde{E}_{i r}(1, \ldots, 1), \quad \tilde{p}_{k}^{\mu}=\tilde{E}_{k}\left(1, \ldots, \sin \chi_{k}, \cos \chi_{k}\right),
$$

and hence

$$
\begin{aligned}
y_{\widetilde{i r} \hat{s}} & =\frac{1}{2} x_{\widetilde{i r}} y(1-\cos \vartheta), \\
y_{\tilde{k} \hat{s}} & =\frac{1}{2} y_{\tilde{k} Q} y\left(1-\sin \chi_{k} \sin \vartheta \cos \varphi-\cos \chi_{k} \cos \vartheta\right),
\end{aligned}
$$

with $\cos \chi_{k}=\cos \chi\left(Y_{\widetilde{i r} \widetilde{k}, Q}\right)$. In this frame, the $y_{\widetilde{i r} \hat{s}}=0$ singularity is at the border of integration. Instead, in the second frame, the $y_{\widetilde{k} \hat{s}}=0$ singularity is at the border,

$$
\tilde{p}_{k}^{\mu}=\tilde{E}_{k}(1, \ldots, 1), \quad \tilde{p}_{i r}^{\mu}=\tilde{E}_{i r}\left(1, \ldots, \sin \chi_{i r}, \cos \chi_{i r}\right),
$$

(note that clearly $\cos \chi_{i r}=\cos \chi_{k}$ ) and hence

$$
\begin{aligned}
y_{\tilde{k} \hat{s}} & =\frac{1}{2} y_{\tilde{k} Q} y(1-\cos \vartheta), \\
y_{\widetilde{i r} \hat{s}} & =\frac{1}{2} x_{\widetilde{i r}} y\left(1-\sin \chi_{i r} \sin \vartheta \cos \varphi-\cos \chi_{i r} \cos \vartheta\right),
\end{aligned}
$$

with $\cos \chi_{i r}=\cos \chi\left(Y_{\widetilde{i r} \widetilde{k}, Q}\right)$.

As for $\mathcal{I}_{2 \mathcal{C S}, 3}^{(l)}$, it is most conveniently evaluated in the frame defined by eq. (E.43), where we can integrate over $\varphi$ using

$$
\frac{\Gamma^{2}(1-\epsilon)}{2 \pi \Gamma(1-2 \epsilon)} \int_{-1}^{1} \mathrm{~d}(\cos \varphi)(\sin \varphi)^{-1-2 \epsilon}=2^{-1-2 \epsilon} .
$$

Then we obtain the following explicit integral representation,

$$
\begin{aligned}
& \mathcal{I}_{2 \mathcal{S S}, 3}^{(l)}\left(x_{\widetilde{i r}} ; \epsilon, \alpha_{0}, y_{0}, d_{0}, d_{0}^{\prime} ; l\right)=-2^{2 \epsilon} \int_{0}^{y_{0}} \mathrm{~d} y y^{-2 \epsilon}(1-y)^{d_{0}^{\prime}-2+\epsilon} \int_{-1}^{1} \mathrm{~d}(\cos \vartheta)(\sin \vartheta)^{-2 \epsilon} \\
& \times\left[(1-y) x_{\widetilde{i r}}+y_{\widetilde{i r} \hat{s}}\right] \int_{0}^{\alpha_{0}} \mathrm{~d} \alpha \alpha^{-\epsilon}(1-\alpha)^{2 d_{0}-3}\left\{\alpha+(1-\alpha)\left[(1-y) x_{\widetilde{i r}}+y_{\widetilde{i r} \hat{s}}\right]\right\}^{-1-\epsilon}
\end{aligned}
$$

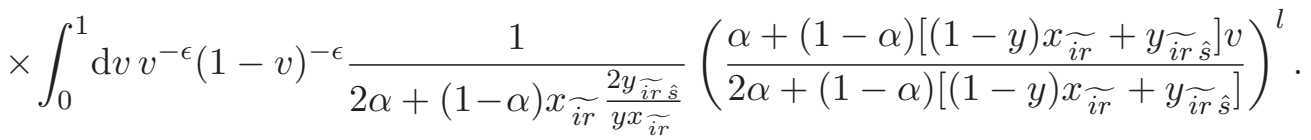


Integrated triple collinear — soft collinear counterterm. For the integral $\mathcal{I}_{2 \mathcal{C S}, 4}^{(l)}$ we use the frame where

$$
\tilde{p}_{i r}^{\mu}=\tilde{E}_{i r}(1, \ldots, 1)
$$

and hence

$$
y_{\widetilde{i r} \hat{s}}=\frac{1}{2} x_{\widetilde{i r}} y(1-\cos \vartheta) .
$$

Then we obtain the following explicit representation,

$$
\begin{aligned}
& \mathcal{I}_{2 C \mathcal{S}, 4}^{(l)}\left(x_{\widetilde{i r}} ; \epsilon, \alpha_{0}, y_{0}, d_{0}, d_{0}^{\prime}\right)=2^{1+2 \epsilon} \int_{0}^{y_{0}} \mathrm{~d} y y^{-1-2 \epsilon}(1-y)^{d_{0}^{\prime}-2+\epsilon} \\
& \quad \times \int_{-1}^{1} \mathrm{~d}(\cos \vartheta)(\sin \vartheta)^{-2 \epsilon}\left[(1-y) x_{\widetilde{i r}}+y_{\widetilde{i r} \hat{s}}\right] \\
& \quad \times \int_{0}^{\alpha_{0}} \mathrm{~d} \alpha \alpha^{-1-\epsilon}(1-\alpha)^{2 d_{0}-3}\left\{\alpha+(1-\alpha)\left[(1-y) x_{\widetilde{i r}}+y_{\widetilde{i r} \hat{s}}\right]\right\}^{-1-\epsilon} \\
& \quad \times \int_{0}^{1} \mathrm{~d} v v^{-\epsilon}(1-v)^{-\epsilon} \frac{2 \alpha+(1-\alpha)\left[(1-y) x_{\widetilde{i r}}+y_{\widetilde{i r} \hat{s}}\right]}{2 \alpha+(1-\alpha) x_{\widetilde{i r}} \frac{2 y_{\widetilde{i r} \hat{s}}}{y x_{\widetilde{i r}}}} \\
& \quad \times\left(\frac{\alpha+(1-\alpha)\left[(1-y) x_{\widetilde{i r}}+y_{\widetilde{i r} \hat{s}}\right] v}{2 \alpha+(1-\alpha)\left[(1-y) x_{\widetilde{i r}}+y_{\widetilde{i r} \hat{s}}\right]}\right)^{l} .
\end{aligned}
$$

Integrated double collinear — soft collinear counterterm. Finally the integral $\mathcal{I}_{2 \mathcal{C S}, 5}^{(l)}$ is most conveniently evaluated in the frame where

$$
\tilde{p}_{j}^{\mu}=\tilde{E}_{j}(1, \ldots, 1), \quad \tilde{p}_{i r}^{\mu}=\tilde{E}_{i r}\left(1, \ldots, \sin \chi_{i r}, \cos \chi_{i r}\right),
$$

and hence

$$
\begin{aligned}
y_{\widetilde{j} \hat{s}} & =\frac{1}{2} y_{\tilde{j} Q} y(1-\cos \vartheta), \\
y_{\widetilde{i r} \hat{s}} & =\frac{1}{2} x_{\widetilde{i r}} y\left(1-\sin \chi_{i r} \sin \vartheta \cos \varphi-\cos \chi_{i r} \cos \vartheta\right),
\end{aligned}
$$

with $\cos \chi_{i r}=\cos \chi\left(Y_{\widetilde{i r} \widetilde{j}, Q}\right)$. The explicit expression for the master integral reads

$$
\begin{aligned}
& \mathcal{I}_{2 \mathcal{C S}, 5}^{(l)}\left(x_{\widetilde{i r}}, Y_{\widetilde{i r} \widetilde{j}, Q} ; \epsilon, \alpha_{0}, y_{0}, d_{0}, d_{0}^{\prime}\right)=\frac{\Gamma^{2}(1-\epsilon)}{2 \pi \Gamma(1-2 \epsilon)} 4 \int_{0}^{y_{0}} \mathrm{~d} y y^{-1-2 \epsilon}(1-y)^{d_{0}^{\prime}-2+\epsilon} \\
& \quad \times \int_{-1}^{1} \mathrm{~d}(\cos \vartheta) \mathrm{d}(\cos \varphi)(\sin \vartheta)^{-2 \epsilon}(\sin \varphi)^{-1-2 \epsilon}\left[(1-y) x_{\widetilde{i r}}+y_{\widetilde{i r} \hat{s}}\right] \\
& \quad \times \int_{0}^{\alpha_{0}} \mathrm{~d} \alpha \alpha^{-1-\epsilon}(1-\alpha)^{2 d_{0}-3}\left\{\alpha+(1-\alpha)\left[(1-y) x_{\widetilde{i r}}+y_{\widetilde{i r} \hat{s}}\right]\right\}^{-1-\epsilon} \\
& \quad \times \int_{0}^{1} \mathrm{~d} v v^{-\epsilon}(1-v)^{-\epsilon} \frac{y y_{\tilde{j} Q}}{2 y_{\tilde{j} \hat{s}}}\left(1-y+\frac{y_{\tilde{j} \hat{s}}}{y_{\tilde{j} Q}}\right)\left(\frac{\alpha+(1-\alpha)\left[(1-y) x_{\widetilde{i r}}+y_{\widetilde{i r} \hat{s}}\right] v}{2 \alpha+(1-\alpha)\left[(1-y) x_{\widetilde{i r}}+y_{\widetilde{i r} \hat{s}}\right]}\right)^{l} .
\end{aligned}
$$

The parametric forms for the integrals $\mathcal{I}_{2 \mathcal{L}, n}^{(l)}$ presented in this section are directly suitable for evaluation using sector decomposition, after introducing the new variables

$$
z_{1}=\frac{1-\cos \vartheta}{2} \quad \text { and } \quad z_{2}=\frac{1-\cos \varphi}{2} .
$$


Open Access. This article is distributed under the terms of the Creative Commons Attribution License which permits any use, distribution and reproduction in any medium, provided the original author(s) and source are credited.

\section{References}

[1] S. Frixione, Z. Kunszt and A. Signer, Three jet cross-sections to next-to-leading order, Nucl. Phys. B 467 (1996) 399 [hep-ph/9512328] [INSPIRE].

[2] S. Catani and M. Seymour, A general algorithm for calculating jet cross-sections in NLO QCD, Nucl. Phys. B 485 (1997) 291 [Erratum ibid. B 510 (1998) 503] [hep-ph/9605323] [INSPIRE].

[3] Z. Nagy and Z. Trócsányi, Calculation of QCD jet cross-sections at next-to-leading order, Nucl. Phys. B 486 (1997) 189 [hep-ph/9610498] [INSPIRE].

[4] G. Somogyi and Z. Trócsányi, A new subtraction scheme for computing QCD jet cross sections at next-to-leading order accuracy, hep-ph/0609041 [INSPIRE].

[5] G. Somogyi, Subtraction with hadronic initial states at NLO: An NNLO-compatible scheme, JHEP 05 (2009) 016 [arXiv:0903.1218] [INSPIRE].

[6] S. Frixione and B.R. Webber, Matching NLO QCD computations and parton shower simulations, JHEP 06 (2002) 029 [hep-ph/0204244] [INSPIRE].

[7] P. Nason and G. Ridolfi, A positive-weight next-to-leading-order Monte Carlo for Z pair hadroproduction, JHEP 08 (2006) 077 [hep-ph/0606275] [INSPIRE].

[8] S. Frixione, P. Nason and G. Ridolfi, A positive-weight next-to-leading-order Monte Carlo for heavy flavour hadroproduction, JHEP 09 (2007) 126 [arXiv:0707.3088] [INSPIRE].

[9] S. Frixione, P. Nason and C. Oleari, Matching NLO QCD computations with Parton Shower simulations: the POWHEG method, JHEP 11 (2007) 070 [arXiv: 0709.2092] [INSPIRE].

[10] K. Melnikov and F. Petriello, Electroweak gauge boson production at hadron colliders through $O\left(\alpha_{s}^{2}\right)$, Phys. Rev. D 74 (2006) 114017 [hep-ph/0609070] [INSPIRE].

[11] S. Catani, L. Cieri, G. Ferrera, D. de Florian and M. Grazzini, Vector boson production at hadron colliders: a fully exclusive QCD calculation at NNLO,

Phys. Rev. Lett. 103 (2009) 082001 [arXiv:0903.2120] [INSPIRE].

[12] C. Anastasiou, K. Melnikov and F. Petriello, Higgs boson production at hadron colliders: Differential cross sections through next-to-next-to-leading order, Phys. Rev. Lett. 93 (2004) 262002 [hep-ph/0409088] [INSPIRE].

[13] S. Catani and M. Grazzini, An NNLO subtraction formalism in hadron collisions and its application to Higgs boson production at the LHC, Phys. Rev. Lett. 98 (2007) 222002 [hep-ph/0703012] [INSPIRE].

[14] S. Catani, L. Cieri, D. de Florian, G. Ferrera and M. Grazzini, Diphoton production at hadron colliders: a fully-differential QCD calculation at NNLO, Phys. Rev. Lett. 108 (2012) 072001 [arXiv:1110.2375] [INSPIRE].

[15] G. Ferrera, M. Grazzini and F. Tramontano, Associated WH production at hadron colliders: a fully exclusive QCD calculation at NNLO, Phys. Rev. Lett. 107 (2011) 152003 [arXiv: 1107.1164] [INSPIRE]. 
[16] P. Baernreuther, M. Czakon and A. Mitov, Percent Level Precision Physics at the Tevatron: First Genuine NNLO QCD Corrections to $q \bar{q} \rightarrow t \bar{t}+X$, Phys. Rev. Lett. 109 (2012) 132001 [arXiv: 1204.5201] [INSPIRE].

[17] M. Czakon and A. Mitov, NNLO corrections to top-pair production at hadron colliders: the all-fermionic scattering channels, JHEP 12 (2012) 054 [arXiv:1207.0236] [INSPIRE].

[18] M. Czakon and A. Mitov, NNLO corrections to top pair production at hadron colliders: the quark-gluon reaction, JHEP 01 (2013) 080 [arXiv: 1210.6832] [INSPIRE].

[19] S. Frixione and M. Grazzini, Subtraction at NNLO, JHEP 06 (2005) 010 [hep-ph/0411399] [INSPIRE].

[20] G. Somogyi, Z. Trócsányi and V. Del Duca, Matching of singly- and doubly-unresolved limits of tree-level QCD squared matrix elements, JHEP 06 (2005) 024 [hep-ph/0502226] [INSPIRE].

[21] A. Gehrmann-De Ridder, T. Gehrmann and E.N. Glover, Antenna subtraction at NNLO, JHEP 09 (2005) 056 [hep-ph/0505111] [INSPIRE].

[22] G. Somogyi, Z. Trócsányi and V. Del Duca, A Subtraction scheme for computing QCD jet cross sections at NNLO: regularization of doubly-real emissions, JHEP 01 (2007) 070 [hep-ph/0609042] [INSPIRE].

[23] G. Somogyi and Z. Trócsányi, A Subtraction scheme for computing QCD jet cross sections at NNLO: Regularization of real-virtual emission, JHEP 01 (2007) 052 [hep-ph/0609043] [INSPIRE].

[24] A. Daleo, T. Gehrmann and D. Maître, Antenna subtraction with hadronic initial states, JHEP 04 (2007) 016 [hep-ph/0612257] [INSPIRE].

[25] G. Somogyi and Z. Trócsányi, A subtraction scheme for computing QCD jet cross sections at NNLO: Integrating the subtraction terms. I., JHEP 08 (2008) 042 [arXiv:0807.0509] [INSPIRE].

[26] U. Aglietti, V. Del Duca, C. Duhr, G. Somogyi and Z. Trócsányi, Analytic integration of real-virtual counterterms in NNLO jet cross sections. I., JHEP 09 (2008) 107 [arXiv: 0807.0514] [INSPIRE].

[27] P. Bolzoni, S.-O. Moch, G. Somogyi and Z. Trócsányi, Analytic integration of real-virtual counterterms in NNLO jet cross sections. II., JHEP 08 (2009) 079 [arXiv:0905.4390] [INSPIRE].

[28] A. Daleo, A. Gehrmann-De Ridder, T. Gehrmann and G. Luisoni, Antenna subtraction at NNLO with hadronic initial states: initial-final configurations, JHEP 01 (2010) 118 [arXiv: 0912.0374] [INSPIRE].

[29] E. Nigel Glover and J. Pires, Antenna subtraction for gluon scattering at NNLO, JHEP 06 (2010) 096 [arXiv: 1003.2824] [INSPIRE].

[30] M. Czakon, A novel subtraction scheme for double-real radiation at NNLO, Phys. Lett. B 693 (2010) 259 [arXiv: 1005. 0274] [INSPIRE].

[31] P. Bolzoni, G. Somogyi and Z. Trócsányi, A subtraction scheme for computing QCD jet cross sections at NNLO: integrating the iterated singly-unresolved subtraction terms, JHEP 01 (2011) 059 [arXiv: 1011.1909] [INSPIRE]. 
[32] G. Abelof and A. Gehrmann-De Ridder, Antenna subtraction for the production of heavy particles at hadron colliders, JHEP 04 (2011) 063 [arXiv:1102.2443] [INSPIRE].

[33] T. Gehrmann and P.F. Monni, Antenna subtraction at NNLO with hadronic initial states: real-virtual initial-initial configurations, JHEP 12 (2011) 049 [arXiv:1107.4037] [INSPIRE].

[34] A. Gehrmann-De Ridder, E. Glover and J. Pires, Real-virtual corrections for gluon scattering at NNLO, JHEP 02 (2012) 141 [arXiv:1112.3613] [INSPIRE].

[35] A. Gehrmann-De Ridder, T. Gehrmann and M. Ritzmann, Antenna subtraction at NNLO with hadronic initial states: double real initial-initial configurations, JHEP 10 (2012) 047 [arXiv:1207.5779] [INSPIRE].

[36] G. Abelof, O. Dekkers and A. Gehrmann-De Ridder, Antenna subtraction with massive fermions at NNLO: Double real initial-final configurations, JHEP 12 (2012) 107 [arXiv: 1210.5059] [INSPIRE].

[37] A. Gehrmann-De Ridder, T. Gehrmann, E. Glover and J. Pires, Double virtual corrections for gluon scattering at NNLO, JHEP 02 (2013) 026 [arXiv:1211.2710] [INSPIRE].

[38] A. Gehrmann-De Ridder, T. Gehrmann, E. Glover and G. Heinrich, Infrared structure of $e^{+} e^{-} \rightarrow 3$ jets at NNLO, JHEP 11 (2007) 058 [arXiv:0710.0346] [INSPIRE].

[39] A. Gehrmann-De Ridder, T. Gehrmann, E. Glover and G. Heinrich, Jet rates in electron-positron annihilation at $O\left(\alpha_{s}^{3}\right)$ in QCD, Phys. Rev. Lett. 100 (2008) 172001 [arXiv: 0802.0813] [INSPIRE].

[40] S. Weinzierl, NNLO corrections to 3-jet observables in electron-positron annihilation, Phys. Rev. Lett. 101 (2008) 162001 [arXiv:0807.3241] [inSPIRE].

[41] S. Weinzierl, The Infrared structure of $e^{+} e^{-} \rightarrow 3$ jets at NNLO reloaded, JHEP 07 (2009) 009 [arXiv:0904.1145] [INSPIRE].

[42] A. Gehrmann-De Ridder, T. Gehrmann, E. Glover and G. Heinrich, Second-order QCD corrections to the thrust distribution, Phys. Rev. Lett. 99 (2007) 132002 [arXiv:0707.1285] [INSPIRE].

[43] A. Gehrmann-De Ridder, T. Gehrmann, E. Glover and G. Heinrich, NNLO corrections to event shapes in $e^{+} e^{-}$annihilation, JHEP 12 (2007) 094 [arXiv:0711.4711] [INSPIRE].

[44] A. Gehrmann-De Ridder, T. Gehrmann, E. Glover and G. Heinrich, NNLO moments of event shapes in $e^{+} e^{-}$annihilation, JHEP 05 (2009) 106 [arXiv:0903.4658] [INSPIRE].

[45] S. Weinzierl, Event shapes and jet rates in electron-positron annihilation at NNLO, JHEP 06 (2009) 041 [arXiv:0904.1077] [INSPIRE].

[46] S. Weinzierl, Moments of event shapes in electron-positron annihilation at NNLO, Phys. Rev. D 80 (2009) 094018 [arXiv: 0909.5056] [INSPIRE].

[47] G. Dissertori et al., First determination of the strong coupling constant using NNLO predictions for hadronic event shapes in $e^{+} e^{-}$annihilations, JHEP 02 (2008) 040 [arXiv:0712.0327] [INSPIRE].

[48] G. Dissertori et al., Precise determination of the strong coupling constant at NNLO in QCD from the three-jet rate in electron-positron annihilation at LEP, Phys. Rev. Lett. 104 (2010) 072002 [arXiv:0910.4283] [INSPIRE]. 
[49] G. Dissertori et al., Determination of the strong coupling constant using matched $N N L O+N L L A$ predictions for hadronic event shapes in $e^{+} e^{-}$annihilations, JHEP 08 (2009) 036 [arXiv:0906.3436] [INSPIRE].

[50] JADE collaboration, J. Schieck, S. Bethke, S. Kluth, C. Pahl and Z. Trócsányi, Measurement of the strong coupling $\alpha_{S}$ from the three-jet rate in $e^{+} e^{-}$-annihilation using JADE data, Eur. Phys. J. C 73 (2013) 2332 [arXiv:1205.3714] [InSPIRE].

[51] G. Somogyi, A subtraction scheme for computing QCD jet cross sections at NNLO: integrating the doubly unresolved subtraction terms, JHEP 04 (2013) 010 [arXiv:1301.3919] [INSPIRE].

[52] S. Catani and M. Grazzini, Infrared factorization of tree level QCD amplitudes at the next-to-next-to-leading order and beyond, Nucl. Phys. B 570 (2000) 287 [hep-ph/9908523] [INSPIRE].

[53] G. Heinrich, Sector decomposition, Int. J. Mod. Phys. A 23 (2008) 1457 [arXiv:0803.4177] [INSPIRE].

[54] Z. Kunszt and D.E. Soper, Calculation of jet cross-sections in hadron collisions at order alpha-S ${ }^{3}$, Phys. Rev. D 46 (1992) 192 [INSPIRE].

[55] S. Catani, The singular behavior of QCD amplitudes at two loop order, Phys. Lett. B 427 (1998) 161 [hep-ph/9802439] [INSPIRE].

[56] J. Carter and G. Heinrich, SecDec: a general program for sector decomposition, Comput. Phys. Commun. 182 (2011) 1566 [arXiv:1011.5493] [InSPIRE].

[57] G. Somogyi, Angular integrals in d dimensions, J. Math. Phys. 52 (2011) 083501 [arXiv: 1101.3557] [INSPIRE].

[58] C. Anastasiou, F. Herzog and A. Lazopoulos, On the factorization of overlapping singularities at NNLO, JHEP 03 (2011) 038 [arXiv:1011.4867] [INSPIRE]. 